STATE OF ALASKA

DEPARTMENT OF NATURAL RESOURCES

Alaska Geologic Materials Center Data Report No. 405

\title{
No. 405
}

Petrotechnical Resources of Alaska and TestAmerica

Laboratories Inc., 2012, Geochemical analyses of oil and gas cuttings from the Brontosaurus \#1, Walakpa \#1, S. Barrow \#14, and S. Barrow \#19 wells

Spreadsheet available for download

Received April 2012

All data reports may be downloaded free of charge from the DGGS website. 


\section{TestAmerica}

THE LEADER IN ENVIRONMENTAL TESTING

\section{ANALYTICAL REPORT}

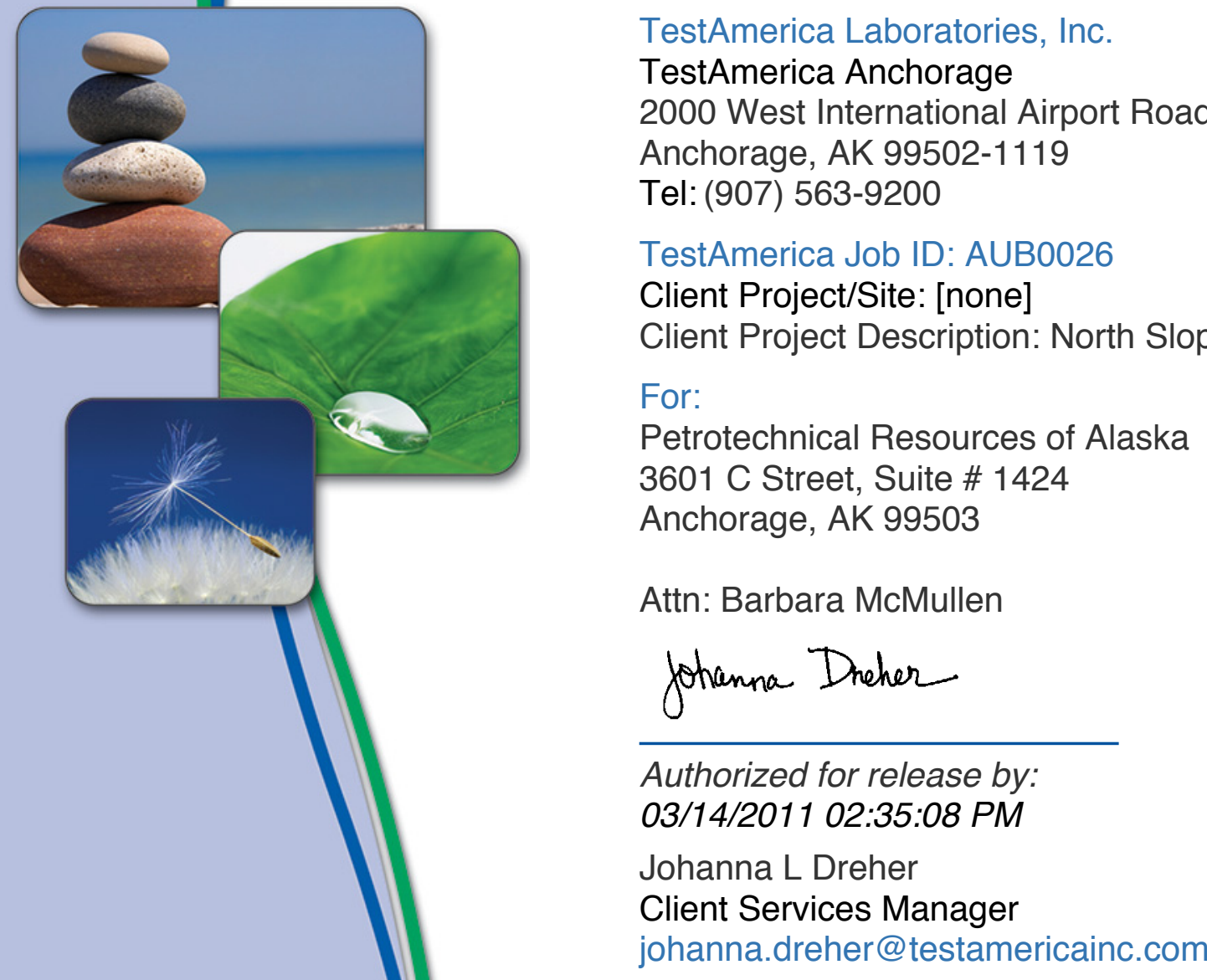

LINKS

Review your project results through

TotalAccess

Have a Question?

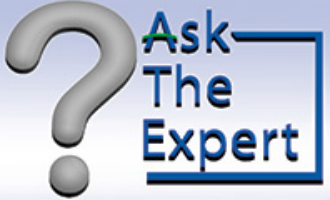

This report has been electronically signed and authorized by the signatory. Electronic signature is intended to be the legally binding equivalent of a traditionally handwritten signature.

Visit us at: 


\section{Table of Contents}

Cover Page . . . . . . . . . . . . . . . . . . 1

Table of Contents ......................... 2

Definitions ............................ 3

Case Narrative . . . . . . . . . . . . . . . . . . . 4

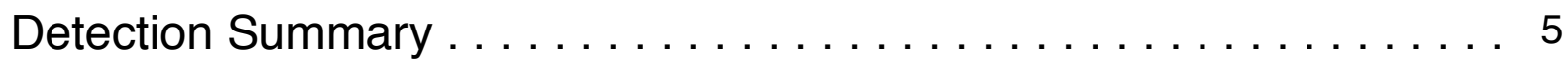

Client Sample Results . . . . . . . . . . . . . . . . . . . 10

QC Sample Results . . . . . . . . . . . . . . . . . . 20

QC Association . . . . . . . . . . . . . . . . . . . 26

Chronicle . . . . . . . . . . . . . . . . . . . . . . . . 31

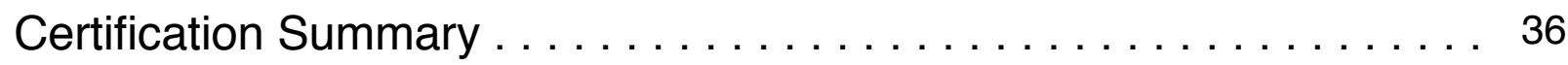

Method Summary . . . . . . . . . . . . . . . . . . . 37

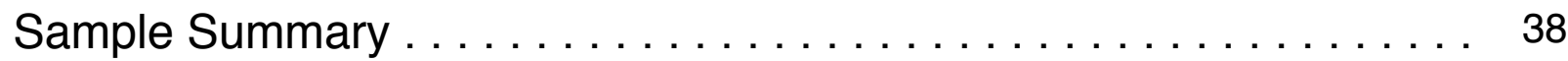

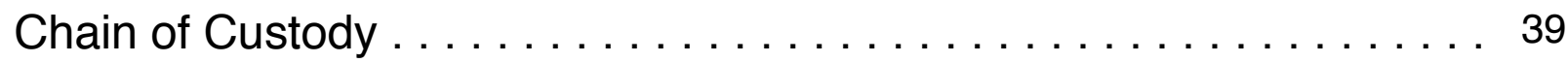




\section{Qualifier Definition/Glossary}

Client: Petrotechnical Resources of Alaska

Project/Site: [none]

\section{Qualifiers}

\section{Metals}

Qualifier Qualifier Description

$\bar{F}$ Duplicate RPD exceeds the control limit

F MS or MSD exceeds the control limits

\section{General Chemistry}

Qualifier Qualifier Description

$\bar{F}$ Duplicate RPD exceeds the control limit

F MS or MSD exceeds the control limits

\section{Glossary}

Glossary Glossary Description

Listed under the "D" column to designate that the result is reported on a dry weight basis. 


\section{Case Narrative}

Client: Petrotechnical Resources of Alaska

Project/Site: [none]

\section{Job ID: AUB0026}

\section{Laboratory: TestAmerica Anchorage}

\section{Narrative}

TestAmerica Anchorage

Receipt

All samples were received in good condition within temperature requirements.

\section{Subcontracted}

This data set was subcontracted to TestAmerica Seattle from TestAmerica Anchorage.

\section{Laboratory: TestAmerica Seattle}

Narrative

Comments

No additional comments.

\section{Receipt}

All samples were received in good condition within temperature requirements.

\section{Metals}

No analytical or quality issues were noted.

\section{General Chemistry - Method $\mathbf{3 0 0 . 0}$}

Due to the high concentration of chloride and sulfate the matrix spike (MS) for batch 81909 could not be evaluated for accuracy and precision. The associated laboratory control sample (LCS) met acceptance criteria.

The matrix spike (MS) recoveries for batch 81909 were outside control limits. The associated laboratory control sample (LCS) recovery met acceptance criteria.

\section{General Chemistry - Method 9012A}

The matrix spike (MS) recoveries for batch 81358 were outside control limits. The associated laboratory control sample (LCS) recovery met acceptance criteria.

No other analytical or quality issues were noted. 


\begin{tabular}{|c|c|c|c|c|c|c|c|c|c|}
\hline Analyte & Result & Qualifier & $\mathbf{R L}$ & MDL & Unit & Dil Fac & D & Method & Prep Type \\
\hline Aluminum & 15000 & & 2800 & & $\overline{\mathrm{mg} / \mathrm{Kg}}$ & 100 & $\bar{z}$ & $\overline{6010 B}$ & Total/NA \\
\hline Iron & 43000 & & 940 & & $\mathrm{mg} / \mathrm{Kg}$ & 100 & 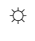 & $6010 \mathrm{~B}$ & Total/NA \\
\hline Magnesium & 9000 & & 5200 & & $\mathrm{mg} / \mathrm{Kg}$ & 100 & 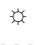 & $6010 B$ & Total/NA \\
\hline Antimony & 0.21 & & 0.17 & & $\mathrm{mg} / \mathrm{Kg}$ & 10 & 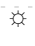 & 6020 & Total/NA \\
\hline Arsenic & 15 & & 0.43 & & $\mathrm{mg} / \mathrm{Kg}$ & 10 & 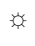 & 6020 & Total/NA \\
\hline Barium & 550 & & 0.17 & & $\mathrm{mg} / \mathrm{Kg}$ & 10 & 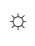 & 6020 & Total/NA \\
\hline Beryllium & 0.95 & & 0.17 & & $\mathrm{mg} / \mathrm{Kg}$ & 10 & 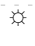 & 6020 & Total/NA \\
\hline Cadmium & 0.44 & & 0.17 & & $\mathrm{mg} / \mathrm{Kg}$ & 10 & 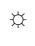 & 6020 & Total/NA \\
\hline Chromium & 54 & & 0.17 & & $\mathrm{mg} / \mathrm{Kg}$ & 10 & 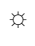 & 6020 & Total/NA \\
\hline Copper & 47 & & 0.17 & & $\mathrm{mg} / \mathrm{Kg}$ & 10 & 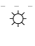 & 6020 & Total/NA \\
\hline Lead & 150 & & 0.17 & & $\mathrm{mg} / \mathrm{Kg}$ & 10 & 列 & 6020 & Total/NA \\
\hline Nickel & 50 & & 0.17 & & $\mathrm{mg} / \mathrm{Kg}$ & 10 & 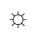 & 6020 & Total/NA \\
\hline Selenium & 0.67 & & 0.60 & & $\mathrm{mg} / \mathrm{Kg}$ & 10 & 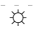 & 6020 & Total/NA \\
\hline Vanadium & 38 & & 0.43 & & $\mathrm{mg} / \mathrm{Kg}$ & 10 & 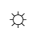 & 6020 & Total/NA \\
\hline Zinc & 120 & & 0.60 & & $\mathrm{mg} / \mathrm{Kg}$ & 10 & 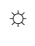 & 6020 & Total/NA \\
\hline Mercury & 0.77 & & 0.017 & & $\mathrm{mg} / \mathrm{Kg}$ & 1 & 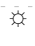 & $7471 \mathrm{~A}$ & Total/NA \\
\hline Chloride & 390 & & 3.3 & & $\mathrm{mg} / \mathrm{Kg}$ & 1 & 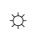 & 300.0 & Soluble \\
\hline Sulfate & 210 & & 3.0 & & $\mathrm{mg} / \mathrm{Kg}$ & 1 & 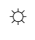 & 300.0 & Soluble \\
\hline
\end{tabular}

\section{Client Sample ID: Brontosaurus \#1 \#2- intermed hole}

Lab Sample ID: AUB0026-02

\begin{tabular}{|c|c|c|c|c|c|c|c|c|c|}
\hline Analyte & Result & Qualifier & $\mathbf{R L}$ & MDL & Unit & Dil Fac & D & Method & Prep Type \\
\hline Aluminum & 13000 & & 2000 & & $\mathrm{mg} / \mathrm{Kg}$ & 100 & $\bar{x}$ & $6010 B$ & Total/NA \\
\hline Iron & 59000 & & 660 & & $\mathrm{mg} / \mathrm{Kg}$ & 100 & x & $6010 \mathrm{~B}$ & Total/NA \\
\hline Magnesium & 9600 & & 3600 & & $\mathrm{mg} / \mathrm{Kg}$ & 100 & 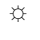 & $6010 \mathrm{~B}$ & Total/NA \\
\hline Antimony & 0.25 & & 0.18 & & $\mathrm{mg} / \mathrm{Kg}$ & 10 & 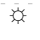 & 6020 & Total/NA \\
\hline Arsenic & 15 & & 0.46 & & $\mathrm{mg} / \mathrm{Kg}$ & 10 & is & 6020 & Total/NA \\
\hline Barium & 240 & & 0.18 & & $\mathrm{mg} / \mathrm{Kg}$ & 10 & 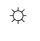 & 6020 & Total/NA \\
\hline Beryllium & 0.90 & & 0.18 & & $\mathrm{mg} / \mathrm{Kg}$ & 10 & 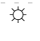 & 6020 & Total/NA \\
\hline Cadmium & 0.26 & & 0.18 & & $\mathrm{mg} / \mathrm{Kg}$ & 10 & 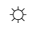 & 6020 & Total/NA \\
\hline Chromium & 36 & & 0.18 & & $\mathrm{mg} / \mathrm{Kg}$ & 10 & 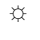 & 6020 & Total/NA \\
\hline Copper & 51 & & 0.18 & & $\mathrm{mg} / \mathrm{Kg}$ & 10 & 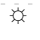 & 6020 & Total/NA \\
\hline Lead & 34 & & 0.18 & & $\mathrm{mg} / \mathrm{Kg}$ & 10 & 列 & 6020 & Total/NA \\
\hline Nickel & 52 & & 0.18 & & $\mathrm{mg} / \mathrm{Kg}$ & 10 & 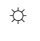 & 6020 & Total/NA \\
\hline Selenium & 0.70 & & 0.64 & & $\mathrm{mg} / \mathrm{Kg}$ & 10 & 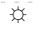 & 6020 & Total/NA \\
\hline Vanadium & 36 & & 0.46 & & $\mathrm{mg} / \mathrm{Kg}$ & 10 & 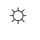 & 6020 & Total/NA \\
\hline Zinc & 110 & & 0.64 & & $\mathrm{mg} / \mathrm{Kg}$ & 10 & 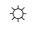 & 6020 & Total/NA \\
\hline Mercury & 0.37 & & 0.013 & & $\mathrm{mg} / \mathrm{Kg}$ & 1 & 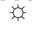 & $7471 \mathrm{~A}$ & Total/NA \\
\hline Chloride & 75 & & 3.3 & & $\mathrm{mg} / \mathrm{Kg}$ & 1 & 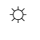 & 300.0 & Soluble \\
\hline Sulfate & 750 & & 30 & & $\mathrm{mg} / \mathrm{Kg}$ & 10 & 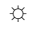 & 300.0 & Soluble \\
\hline
\end{tabular}

Client Sample ID: Brontosaurus \#1 \#3- intermed hole

Lab Sample ID: AUB0026-03

\begin{tabular}{|c|c|c|c|c|c|c|c|c|c|}
\hline Analyte & Result & Qualifier & $\mathbf{R L}$ & MDL & Unit & Dil Fac & D & Method & Prep Type \\
\hline Aluminum & 11000 & & 2400 & & $\mathrm{mg} / \mathrm{Kg}$ & 100 & $\bar{x}$ & $\overline{6010 B}$ & Total/NA \\
\hline Iron & 40000 & & 800 & & $\mathrm{mg} / \mathrm{Kg}$ & 100 & 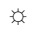 & $6010 \mathrm{~B}$ & Total/NA \\
\hline Magnesium & 8000 & & 4400 & & $\mathrm{mg} / \mathrm{Kg}$ & 100 & 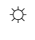 & $6010 B$ & Total/NA \\
\hline Antimony & 0.36 & & 0.15 & & $\mathrm{mg} / \mathrm{Kg}$ & 10 & 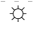 & 6020 & Total/NA \\
\hline Arsenic & 21 & & 0.38 & & $\mathrm{mg} / \mathrm{Kg}$ & 10 & 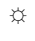 & 6020 & Total/NA \\
\hline Barium & 150 & & 0.15 & & $\mathrm{mg} / \mathrm{Kg}$ & 10 & 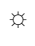 & 6020 & Total/NA \\
\hline Beryllium & 0.86 & & 0.15 & & $\mathrm{mg} / \mathrm{Kg}$ & 10 & 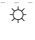 & 6020 & Total/NA \\
\hline Cadmium & 0.98 & & 0.15 & & $\mathrm{mg} / \mathrm{Kg}$ & 10 & 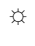 & 6020 & Total/NA \\
\hline Chromium & 27 & & 0.15 & & $\mathrm{mg} / \mathrm{Kg}$ & 10 & 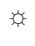 & 6020 & Total/NA \\
\hline Copper & 47 & & 0.15 & & $\mathrm{mg} / \mathrm{Kg}$ & 10 & - & 6020 & Total/NA \\
\hline
\end{tabular}




\begin{tabular}{|c|c|c|c|c|c|c|c|c|c|}
\hline Analyte & Result & Qualifier & $\mathbf{R L}$ & MDL & Unit & Dil Fac & D & Method & Prep Type \\
\hline Lead & 74 & & 0.15 & & $\mathrm{mg} / \mathrm{Kg}$ & 10 & $\bar{z}$ & 6020 & Total/NA \\
\hline Nickel & 53 & & 0.15 & & $\mathrm{mg} / \mathrm{Kg}$ & 10 & 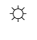 & 6020 & Total/NA \\
\hline Selenium & 3.8 & & 0.53 & & $\mathrm{mg} / \mathrm{Kg}$ & 10 & 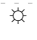 & 6020 & Total/NA \\
\hline Silver & 0.29 & & 0.15 & & $\mathrm{mg} / \mathrm{Kg}$ & 10 & 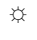 & 6020 & Total/NA \\
\hline Thallium & 0.33 & & 0.30 & & $\mathrm{mg} / \mathrm{Kg}$ & 10 & 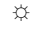 & 6020 & Total/NA \\
\hline Vanadium & 40 & & 0.38 & & $\mathrm{mg} / \mathrm{Kg}$ & 10 & 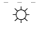 & 6020 & Total/NA \\
\hline Zinc & 140 & & 0.53 & & $\mathrm{mg} / \mathrm{Kg}$ & 10 & 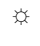 & 6020 & Total/NA \\
\hline Mercury & 0.72 & & 0.016 & & $\mathrm{mg} / \mathrm{Kg}$ & 1 & 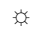 & $7471 \mathrm{~A}$ & Total/NA \\
\hline Chloride & 61 & & 3.3 & & $\mathrm{mg} / \mathrm{Kg}$ & 1 & 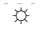 & 300.0 & Soluble \\
\hline Sulfate & 500 & & 3.0 & & $\mathrm{mg} / \mathrm{Kg}$ & 1 & 好 & 300.0 & Soluble \\
\hline
\end{tabular}

Client Sample ID: Walakpa \#1 \#1- surface hole

Lab Sample ID: AUB0026-04

\begin{tabular}{|c|c|c|c|c|c|c|c|c|c|}
\hline Analyte & Result & Qualifier & RL & MDL & Unit & Dil Fac & D & Method & Prep Type \\
\hline Aluminum & 14000 & & 2600 & & $\mathrm{mg} / \mathrm{Kg}$ & $\overline{100}$ & $\bar{x}$ & $6010 B$ & Total/NA \\
\hline Iron & 40000 & & 860 & & $\mathrm{mg} / \mathrm{Kg}$ & 100 & 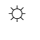 & $6010 \mathrm{~B}$ & Total/NA \\
\hline Magnesium & 10000 & & 4700 & & $\mathrm{mg} / \mathrm{Kg}$ & 100 & $x$ & $6010 B$ & Total/NA \\
\hline Antimony & 0.19 & & 0.16 & & $\mathrm{mg} / \mathrm{Kg}$ & 10 & - & 6020 & Total/NA \\
\hline Arsenic & 15 & & 0.41 & & $\mathrm{mg} / \mathrm{Kg}$ & 10 & $x$ & 6020 & Total/NA \\
\hline Barium & 200 & & 0.16 & & $\mathrm{mg} / \mathrm{Kg}$ & 10 & 努 & 6020 & Total/NA \\
\hline Beryllium & 0.98 & & 0.16 & & $\mathrm{mg} / \mathrm{Kg}$ & 10 & 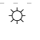 & 6020 & Total/NA \\
\hline Cadmium & 0.37 & & 0.16 & & $\mathrm{mg} / \mathrm{Kg}$ & 10 & $x$ & 6020 & Total/NA \\
\hline Chromium & 31 & & 0.16 & & $\mathrm{mg} / \mathrm{Kg}$ & 10 & 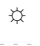 & 6020 & Total/NA \\
\hline Copper & 41 & & 0.16 & & $\mathrm{mg} / \mathrm{Kg}$ & 10 & 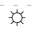 & 6020 & Total/NA \\
\hline Lead & 240 & & 0.16 & & $\mathrm{mg} / \mathrm{Kg}$ & 10 & 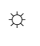 & 6020 & Total/NA \\
\hline Nickel & 48 & & 0.16 & & $\mathrm{mg} / \mathrm{Kg}$ & 10 & $x$ & 6020 & Total/NA \\
\hline Selenium & 0.80 & & 0.57 & & $\mathrm{mg} / \mathrm{Kg}$ & 10 & 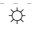 & 6020 & Total/NA \\
\hline Vanadium & 39 & & 0.41 & & $\mathrm{mg} / \mathrm{Kg}$ & 10 & 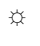 & 6020 & Total/NA \\
\hline Zinc & 230 & & 0.57 & & $\mathrm{mg} / \mathrm{Kg}$ & 10 & 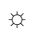 & 6020 & Total/NA \\
\hline Mercury & 0.31 & & 0.013 & & $\mathrm{mg} / \mathrm{Kg}$ & 1 & 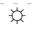 & $7471 \mathrm{~A}$ & Total/NA \\
\hline Chloride & 460 & & 3.3 & & $\mathrm{mg} / \mathrm{Kg}$ & 1 & 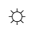 & 300.0 & Soluble \\
\hline Sulfate & 1400 & & 30 & & $\mathrm{mg} / \mathrm{Kg}$ & 10 & $x$ & 300.0 & Soluble \\
\hline
\end{tabular}

Client Sample ID: Walakpa \#1 \#2- intermed. hole

Lab Sample ID: AUB0026-05

\begin{tabular}{|c|c|c|c|c|c|c|c|c|c|}
\hline Analyte & Result & Qualifier & RL & MDL & Unit & Dil Fac & D & Method & Prep Type \\
\hline Aluminum & 14000 & & 2100 & & $\mathrm{mg} / \mathrm{Kg}$ & 100 & $\bar{x}$ & $\overline{6010 B}$ & Total/NA \\
\hline Iron & 47000 & & 700 & & $\mathrm{mg} / \mathrm{Kg}$ & 100 & 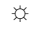 & $6010 \mathrm{~B}$ & Total/NA \\
\hline Magnesium & 9600 & & 3900 & & $\mathrm{mg} / \mathrm{Kg}$ & 100 & $x$ & $6010 \mathrm{~B}$ & Total/NA \\
\hline Antimony & 0.17 & & 0.15 & & $\mathrm{mg} / \mathrm{Kg}$ & 10 & 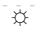 & 6020 & Total/NA \\
\hline Arsenic & 12 & & 0.37 & & $\mathrm{mg} / \mathrm{Kg}$ & 10 & 家 & 6020 & Total/NA \\
\hline Barium & 170 & & 0.15 & & $\mathrm{mg} / \mathrm{Kg}$ & 10 & 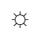 & 6020 & Total/NA \\
\hline Beryllium & 0.91 & & 0.15 & & $\mathrm{mg} / \mathrm{Kg}$ & 10 & 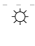 & 6020 & Total/NA \\
\hline Cadmium & 0.31 & & 0.15 & & $\mathrm{mg} / \mathrm{Kg}$ & 10 & 要 & 6020 & Total/NA \\
\hline Chromium & 30 & & 0.15 & & $\mathrm{mg} / \mathrm{Kg}$ & 10 & 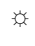 & 6020 & Total/NA \\
\hline Copper & 36 & & 0.15 & & $\mathrm{mg} / \mathrm{Kg}$ & 10 & 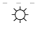 & 6020 & Total/NA \\
\hline Lead & 130 & & 0.15 & & $\mathrm{mg} / \mathrm{Kg}$ & 10 & 齐 & 6020 & Total/NA \\
\hline Nickel & 46 & & 0.15 & & $\mathrm{mg} / \mathrm{Kg}$ & 10 & 家 & 6020 & Total/NA \\
\hline Selenium & 0.74 & & 0.52 & & $\mathrm{mg} / \mathrm{Kg}$ & 10 & 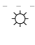 & 6020 & Total/NA \\
\hline Vanadium & 34 & & 0.37 & & $\mathrm{mg} / \mathrm{Kg}$ & 10 & 家 & 6020 & Total/NA \\
\hline Zinc & 340 & & 0.52 & & $\mathrm{mg} / \mathrm{Kg}$ & 10 & 究 & 6020 & Total/NA \\
\hline Mercury & 0.66 & & 0.12 & & $\mathrm{mg} / \mathrm{Kg}$ & 10 & 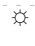 & $7471 \mathrm{~A}$ & Total/NA \\
\hline Chloride & 140 & & 3.3 & & $\mathrm{mg} / \mathrm{Kg}$ & 1 & 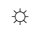 & 300.0 & Soluble \\
\hline
\end{tabular}


Analyte

Sulfate
Result Qualifier

410
RL

$\bar{R}$
MDL Unit

Unit
Dil Fac D Method

$1 \frac{}{300.0}$
Prep Type

Soluble

\section{Client Sample ID: Walakpa \#1 \#3- intermed. hole}

\begin{tabular}{lrr} 
Analyte & Result & Qualifier \\
\cline { 1 - 2 } Aluminum & 12000 \\
Iron & 42000 \\
Magnesium & 7600 \\
Antimony & 0.33 \\
Arsenic & 21 \\
Barium & 220 \\
Beryllium & 0.70 \\
Cadmium & 0.58 \\
Chromium & 23 \\
Copper & 47 \\
Lead & 86 \\
Nickel & 48 \\
Selenium & 4.2 \\
Silver & 0.29 \\
Vanadium & 45 \\
Zinc & 150 \\
Mercury & 0.49 \\
Chloride & 82 \\
Sulfate & 1100
\end{tabular}

Lab Sample ID: AUB0026-06

\begin{tabular}{|c|c|c|}
\hline RL & MDL & Unit \\
\hline 2900 & & $\mathrm{mg} / \mathrm{Kg}$ \\
\hline 960 & & $\mathrm{mg} / \mathrm{Kg}$ \\
\hline 5300 & & $\mathrm{mg} / \mathrm{Kg}$ \\
\hline 0.16 & & $\mathrm{mg} / \mathrm{Kg}$ \\
\hline 0.39 & & $\mathrm{mg} / \mathrm{Kg}$ \\
\hline 0.16 & & $\mathrm{mg} / \mathrm{Kg}$ \\
\hline 0.16 & & $\mathrm{mg} / \mathrm{Kg}$ \\
\hline 0.16 & & $\mathrm{mg} / \mathrm{Kg}$ \\
\hline 0.16 & & $\mathrm{mg} / \mathrm{Kg}$ \\
\hline 0.16 & & $\mathrm{mg} / \mathrm{Kg}$ \\
\hline 0.16 & & $\mathrm{mg} / \mathrm{Kg}$ \\
\hline 0.16 & & $\mathrm{mg} / \mathrm{Kg}$ \\
\hline 0.55 & & $\mathrm{mg} / \mathrm{Kg}$ \\
\hline 0.16 & & $\mathrm{mg} / \mathrm{Kg}$ \\
\hline 0.39 & & $\mathrm{mg} / \mathrm{Kg}$ \\
\hline 0.55 & & $\mathrm{mg} / \mathrm{Kg}$ \\
\hline 0.015 & & $\mathrm{mg} / \mathrm{Kg}$ \\
\hline 3.3 & & $\mathrm{mg} / \mathrm{Kg}$ \\
\hline 30 & & $\mathrm{mg} / \mathrm{Kg}$ \\
\hline
\end{tabular}

Dil

Client Sample ID: S. Barrow 19 \#1- surface hole Lab Sample ID: AUB0026-07

\begin{tabular}{|c|c|c|c|c|c|c|c|c|c|}
\hline Analyte & Result & Qualifier & RL & MDL & Unit & Dil Fac & D & Method & Prep Type \\
\hline Aluminum & 14000 & & 2500 & & $\mathrm{mg} / \mathrm{Kg}$ & 100 & 栾 & 6010B & Total/NA \\
\hline Iron & 41000 & & 840 & & $\mathrm{mg} / \mathrm{Kg}$ & 100 & 媇 & $6010 \mathrm{~B}$ & Total/NA \\
\hline Magnesium & 9600 & & 4600 & & $\mathrm{mg} / \mathrm{Kg}$ & 100 & 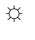 & $6010 B$ & Total/NA \\
\hline Antimony & 0.34 & & 0.17 & & $\mathrm{mg} / \mathrm{Kg}$ & 10 & 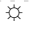 & 6020 & Total/NA \\
\hline Arsenic & 18 & & 0.42 & & $\mathrm{mg} / \mathrm{Kg}$ & 10 & 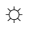 & 6020 & Total/NA \\
\hline Barium & 800 & & 0.17 & & $\mathrm{mg} / \mathrm{Kg}$ & 10 & 沓 & 6020 & Total/NA \\
\hline Beryllium & 0.98 & & 0.17 & & $\mathrm{mg} / \mathrm{Kg}$ & 10 & 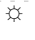 & 6020 & Total/NA \\
\hline Cadmium & 0.32 & & 0.17 & & $\mathrm{mg} / \mathrm{Kg}$ & 10 & 安 & 6020 & Total/NA \\
\hline Chromium & 26 & & 0.17 & & $\mathrm{mg} / \mathrm{Kg}$ & 10 & 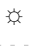 & 6020 & Total/NA \\
\hline Copper & 30 & & 0.17 & & $\mathrm{mg} / \mathrm{Kg}$ & 10 & 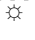 & 6020 & Total/NA \\
\hline Lead & 230 & & 0.17 & & $\mathrm{mg} / \mathrm{Kg}$ & 10 & 济 & 6020 & Total/NA \\
\hline Nickel & 39 & & 0.17 & & $\mathrm{mg} / \mathrm{Kg}$ & 10 & 悾 & 6020 & Total/NA \\
\hline Selenium & 0.63 & & 0.59 & & $\mathrm{mg} / \mathrm{Kg}$ & 10 & 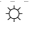 & 6020 & Total/NA \\
\hline Vanadium & 36 & & 0.42 & & $\mathrm{mg} / \mathrm{Kg}$ & 10 & 说 & 6020 & Total/NA \\
\hline Zinc & 200 & & 0.59 & & $\mathrm{mg} / \mathrm{Kg}$ & 10 & 次 & 6020 & Total/NA \\
\hline Mercury & 0.71 & & 0.14 & & $\mathrm{mg} / \mathrm{Kg}$ & 10 & 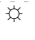 & $7471 \mathrm{~A}$ & Total/NA \\
\hline Chloride & 750 & & 33 & & $\mathrm{mg} / \mathrm{Kg}$ & 10 & 沓 & 300.0 & Soluble \\
\hline Sulfate & 3300 & & 30 & & $\mathrm{mg} / \mathrm{Kg}$ & 10 & 好 & 300.0 & Soluble \\
\hline
\end{tabular}

Client Sample ID: S. Barrow 19 \#2- intermed. hole

Lab Sample ID: AUB0026-08

\begin{tabular}{|c|c|c|c|c|c|c|c|c|c|}
\hline Analyte & Result & Qualifier & RL & MDL & Unit & Dil Fac & D & Method & Prep Type \\
\hline Aluminum & 9100 & & 2500 & & $\mathrm{mg} / \mathrm{Kg}$ & 100 & $\bar{\phi}$ & $6010 B$ & Total/NA \\
\hline Iron & 45000 & & 830 & & $\mathrm{mg} / \mathrm{Kg}$ & 100 & 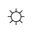 & $6010 B$ & Total/NA \\
\hline Magnesium & 8300 & & 4600 & & $\mathrm{mg} / \mathrm{Kg}$ & 100 & 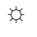 & $6010 \mathrm{~B}$ & Total/NA \\
\hline Antimony & 0.62 & & 0.19 & & $\mathrm{mg} / \mathrm{Kg}$ & 10 & 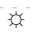 & 6020 & Total/NA \\
\hline
\end{tabular}




\begin{tabular}{|c|c|c|c|c|c|c|c|c|c|}
\hline Analyte & Result & Qualifier & $\mathbf{R L}$ & MDL & Unit & Dil Fac & D & Method & Prep Type \\
\hline Arsenic & 36 & & 0.48 & & $\mathrm{mg} / \mathrm{Kg}$ & 10 & $\bar{x}$ & $\overline{6020}$ & Total/NA \\
\hline Barium & 140 & & 0.19 & & $\mathrm{mg} / \mathrm{Kg}$ & 10 & 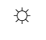 & 6020 & Total/NA \\
\hline Beryllium & 0.63 & & 0.19 & & $\mathrm{mg} / \mathrm{Kg}$ & 10 & 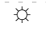 & 6020 & Total/NA \\
\hline Cadmium & 0.38 & & 0.19 & & $\mathrm{mg} / \mathrm{Kg}$ & 10 & $x$ & 6020 & Total/NA \\
\hline Chromium & 120 & & 0.19 & & $\mathrm{mg} / \mathrm{Kg}$ & 10 & 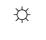 & 6020 & Total/NA \\
\hline Copper & 44 & & 0.19 & & $\mathrm{mg} / \mathrm{Kg}$ & 10 & 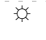 & 6020 & Total/NA \\
\hline Lead & 2400 & & 19 & & $\mathrm{mg} / \mathrm{Kg}$ & 1000 & 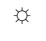 & 6020 & Total/NA \\
\hline Nickel & 53 & & 0.19 & & $\mathrm{mg} / \mathrm{Kg}$ & 10 & 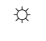 & 6020 & Total/NA \\
\hline Selenium & 3.2 & & 0.68 & & $\mathrm{mg} / \mathrm{Kg}$ & 10 & 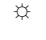 & 6020 & Total/NA \\
\hline Silver & 0.37 & & 0.19 & & $\mathrm{mg} / \mathrm{Kg}$ & 10 & 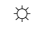 & 6020 & Total/NA \\
\hline Vanadium & 46 & & 0.48 & & $\mathrm{mg} / \mathrm{Kg}$ & 10 & 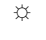 & 6020 & Total/NA \\
\hline Zinc & 640 & & 0.68 & & $\mathrm{mg} / \mathrm{Kg}$ & 10 & 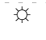 & 6020 & Total/NA \\
\hline Mercury & 0.92 & & 0.16 & & $\mathrm{mg} / \mathrm{Kg}$ & 10 & 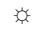 & $7471 \mathrm{~A}$ & Total/NA \\
\hline Chloride & 11000 & & 170 & & $\mathrm{mg} / \mathrm{Kg}$ & 50 & 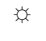 & 300.0 & Soluble \\
\hline Sulfate & 11000 & & 150 & & $\mathrm{mg} / \mathrm{Kg}$ & 50 & 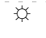 & 300.0 & Soluble \\
\hline
\end{tabular}

Client Sample ID: S. Barrow 19 \#3- intermed. hole

Lab Sample ID: AUB0026-09

\begin{tabular}{|c|c|c|c|c|c|c|c|c|c|}
\hline Analyte & Result & Qualifier & $\mathbf{R L}$ & MDL & Unit & Dil Fac & D & Method & Prep Type \\
\hline Aluminum & 8500 & & 2500 & & $\mathrm{mg} / \mathrm{Kg}$ & 100 & $\bar{x}$ & $\overline{6010 B}$ & Total/NA \\
\hline Iron & 44000 & & 830 & & $\mathrm{mg} / \mathrm{Kg}$ & 100 & 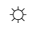 & $6010 \mathrm{~B}$ & Total/NA \\
\hline Magnesium & 5600 & & 4600 & & $\mathrm{mg} / \mathrm{Kg}$ & 100 & $x$ & $6010 \mathrm{~B}$ & Total/NA \\
\hline Antimony & 0.32 & & 0.17 & & $\mathrm{mg} / \mathrm{Kg}$ & 10 & 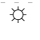 & 6020 & Total/NA \\
\hline Arsenic & 21 & & 0.42 & & $\mathrm{mg} / \mathrm{Kg}$ & 10 & 浆 & 6020 & Total/NA \\
\hline Barium & 140 & & 0.17 & & $\mathrm{mg} / \mathrm{Kg}$ & 10 & 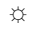 & 6020 & Total/NA \\
\hline Beryllium & 0.59 & & 0.17 & & $\mathrm{mg} / \mathrm{Kg}$ & 10 & 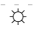 & 6020 & Total/NA \\
\hline Chromium & 49 & & 0.17 & & $\mathrm{mg} / \mathrm{Kg}$ & 10 & x & 6020 & Total/NA \\
\hline Copper & 14 & & 0.17 & & $\mathrm{mg} / \mathrm{Kg}$ & 10 & 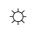 & 6020 & Total/NA \\
\hline Lead & 39 & & 0.17 & & $\mathrm{mg} / \mathrm{Kg}$ & 10 & 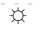 & 6020 & Total/NA \\
\hline Nickel & 34 & & 0.17 & & $\mathrm{mg} / \mathrm{Kg}$ & 10 & t & 6020 & Total/NA \\
\hline Selenium & 0.99 & & 0.59 & & $\mathrm{mg} / \mathrm{Kg}$ & 10 & 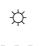 & 6020 & Total/NA \\
\hline Vanadium & 33 & & 0.42 & & $\mathrm{mg} / \mathrm{Kg}$ & 10 & 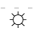 & 6020 & Total/NA \\
\hline Zinc & 100 & & 0.59 & & $\mathrm{mg} / \mathrm{Kg}$ & 10 & 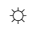 & 6020 & Total/NA \\
\hline Mercury & 0.65 & & 0.013 & & $\mathrm{mg} / \mathrm{Kg}$ & 1 & 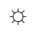 & $7471 \mathrm{~A}$ & Total/NA \\
\hline Chloride & 12000 & & 330 & & $\mathrm{mg} / \mathrm{Kg}$ & 100 & 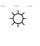 & 300.0 & Soluble \\
\hline Sulfate & 5800 & & 300 & & $\mathrm{mg} / \mathrm{Kg}$ & 100 & 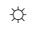 & 300.0 & Soluble \\
\hline
\end{tabular}

Client Sample ID: S. Barrow 14 \#1- surface hole

Lab Sample ID: AUB0026-10

\begin{tabular}{|c|c|c|c|c|c|c|c|c|c|}
\hline Analyte & Result & Qualifier & RL & MDL & Unit & Dil Fac & D & Method & Prep Type \\
\hline Aluminum & 12000 & & 2200 & & $\overline{\mathrm{mg} / \mathrm{Kg}}$ & 100 & $\bar{x}$ & $\overline{6010 B}$ & Total/NA \\
\hline Iron & 53000 & & 750 & & $\mathrm{mg} / \mathrm{Kg}$ & 100 & 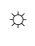 & $6010 \mathrm{~B}$ & Total/NA \\
\hline Magnesium & 7900 & & 4100 & & $\mathrm{mg} / \mathrm{Kg}$ & 100 & ; & $6010 \mathrm{~B}$ & Total/NA \\
\hline Antimony & 0.44 & & 0.18 & & $\mathrm{mg} / \mathrm{Kg}$ & 10 & 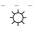 & 6020 & Total/NA \\
\hline Arsenic & 60 & & 0.44 & & $\mathrm{mg} / \mathrm{Kg}$ & 10 & 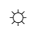 & 6020 & Total/NA \\
\hline Barium & 300 & & 0.18 & & $\mathrm{mg} / \mathrm{Kg}$ & 10 & $x$ & 6020 & Total/NA \\
\hline Beryllium & 0.70 & & 0.18 & & $\mathrm{mg} / \mathrm{Kg}$ & 10 & 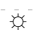 & 6020 & Total/NA \\
\hline Chromium & 40 & & 0.18 & & $\mathrm{mg} / \mathrm{Kg}$ & 10 & 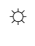 & 6020 & Total/NA \\
\hline Copper & 77 & & 0.18 & & $\mathrm{mg} / \mathrm{Kg}$ & 10 & 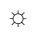 & 6020 & Total/NA \\
\hline Lead & 830 & & 0.18 & & $\mathrm{mg} / \mathrm{Kg}$ & 10 & 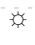 & 6020 & Total/NA \\
\hline Nickel & 55 & & 0.18 & & $\mathrm{mg} / \mathrm{Kg}$ & 10 & 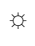 & 6020 & Total/NA \\
\hline Selenium & 1.1 & & 0.61 & & $\mathrm{mg} / \mathrm{Kg}$ & 10 & 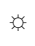 & 6020 & Total/NA \\
\hline Silver & 0.19 & & 0.18 & & $\mathrm{mg} / \mathrm{Kg}$ & 10 & 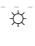 & 6020 & Total/NA \\
\hline Vanadium & 34 & & 0.44 & & $\mathrm{mg} / \mathrm{Kg}$ & 10 & 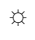 & 6020 & Total/NA \\
\hline
\end{tabular}




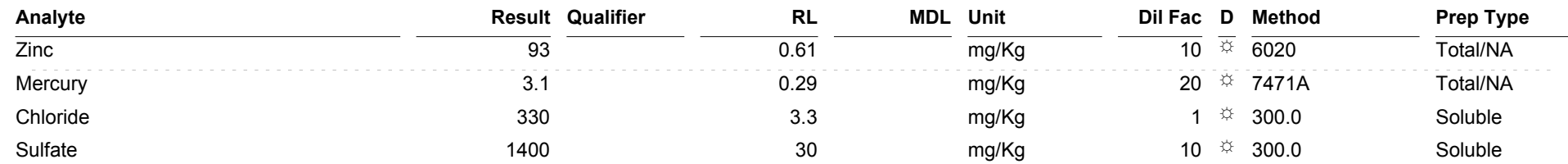

Client Sample ID: S. Barrow 14 \#2- intermed. hole

Lab Sample ID: AUB0026-11

\begin{tabular}{|c|c|c|c|c|c|c|c|c|c|}
\hline Analyte & Result & Qualifier & $\mathbf{R L}$ & MDL & Unit & Dil Fac & D & Method & Prep Type \\
\hline Aluminum & 5800 & & 2300 & & $\mathrm{mg} / \mathrm{Kg}$ & 100 & $\bar{s}$ & $\overline{6010 B}$ & $\overline{\text { Total/NA }}$ \\
\hline Iron & 160000 & & 760 & & $\mathrm{mg} / \mathrm{Kg}$ & 100 & 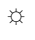 & $6010 \mathrm{~B}$ & Total/NA \\
\hline Magnesium & 7500 & & 4200 & & $\mathrm{mg} / \mathrm{Kg}$ & 100 & 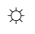 & $6010 \mathrm{~B}$ & Total/NA \\
\hline Antimony & 0.70 & & 0.20 & & $\mathrm{mg} / \mathrm{Kg}$ & 10 & 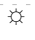 & 6020 & Total/NA \\
\hline Arsenic & 41 & & 0.49 & & $\mathrm{mg} / \mathrm{Kg}$ & 10 & $x$ & 6020 & Total/NA \\
\hline Barium & 190 & & 0.20 & & $\mathrm{mg} / \mathrm{Kg}$ & 10 & $x$ & 6020 & Total/NA \\
\hline Beryllium & 0.63 & & 0.20 & & $\mathrm{mg} / \mathrm{Kg}$ & 10 & 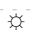 & 6020 & Total/NA \\
\hline Cadmium & 0.48 & & 0.20 & & $\mathrm{mg} / \mathrm{Kg}$ & 10 & 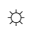 & 6020 & Total/NA \\
\hline Chromium & 82 & & 0.20 & & $\mathrm{mg} / \mathrm{Kg}$ & 10 & 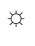 & 6020 & Total/NA \\
\hline Copper & 93 & & 0.20 & & $\mathrm{mg} / \mathrm{Kg}$ & 10 & 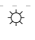 & 6020 & Total/NA \\
\hline Lead & 2800 & & 20 & & $\mathrm{mg} / \mathrm{Kg}$ & 1000 & 永 & 6020 & Total/NA \\
\hline Nickel & 86 & & 0.20 & & $\mathrm{mg} / \mathrm{Kg}$ & 10 & $x$ & 6020 & Total/NA \\
\hline Selenium & 3.1 & & 0.69 & & $\mathrm{mg} / \mathrm{Kg}$ & 10 & 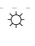 & 6020 & Total/NA \\
\hline Silver & 0.35 & & 0.20 & & $\mathrm{mg} / \mathrm{Kg}$ & 10 & $x$ & 6020 & Total/NA \\
\hline Vanadium & 38 & & 0.49 & & $\mathrm{mg} / \mathrm{Kg}$ & 10 & 家 & 6020 & Total/NA \\
\hline Zinc & 380 & & 0.69 & & $\mathrm{mg} / \mathrm{Kg}$ & 10 & 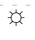 & 6020 & Total/NA \\
\hline Mercury & 1.9 & & 0.13 & & $\mathrm{mg} / \mathrm{Kg}$ & 10 & 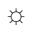 & $7471 \mathrm{~A}$ & Total/NA \\
\hline Chloride & 190 & & 3.3 & & $\mathrm{mg} / \mathrm{Kg}$ & 1 & 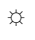 & 300.0 & Soluble \\
\hline Sulfate & 6200 & & 150 & & $\mathrm{mg} / \mathrm{Kg}$ & 50 & 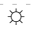 & 300.0 & Soluble \\
\hline
\end{tabular}

Client Sample ID: S. Barrow 14 \#3- intermed. hole

Lab Sample ID: AUB0026-12

\begin{tabular}{|c|c|c|c|c|c|c|c|c|c|}
\hline Analyte & Result & Qualifier & RL & MDL & Unit & Dil Fac & D & Method & Prep Type \\
\hline Aluminum & 4200 & & 2300 & & $\mathrm{mg} / \mathrm{Kg}$ & 100 & 案 & 6010B & Total/NA \\
\hline Iron & 58000 & & 780 & & $\mathrm{mg} / \mathrm{Kg}$ & 100 & 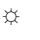 & $6010 B$ & Total/NA \\
\hline Magnesium & 5300 & & 4300 & & $\mathrm{mg} / \mathrm{Kg}$ & 100 & 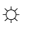 & $6010 B$ & Total/NA \\
\hline Antimony & 0.40 & & 0.17 & & $\mathrm{mg} / \mathrm{Kg}$ & 10 & 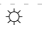 & 6020 & Total/NA \\
\hline Arsenic & 41 & & 0.42 & & $\mathrm{mg} / \mathrm{Kg}$ & 10 & ; & 6020 & Total/NA \\
\hline Barium & 75 & & 0.17 & & $\mathrm{mg} / \mathrm{Kg}$ & 10 & 努 & 6020 & Total/NA \\
\hline Beryllium & 0.45 & & 0.17 & & $\mathrm{mg} / \mathrm{Kg}$ & 10 & 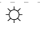 & 6020 & Total/NA \\
\hline Chromium & 60 & & 0.17 & & $\mathrm{mg} / \mathrm{Kg}$ & 10 & 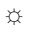 & 6020 & Total/NA \\
\hline Copper & 35 & & 0.17 & & $\mathrm{mg} / \mathrm{Kg}$ & 10 & 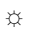 & 6020 & Total/NA \\
\hline Lead & 110 & & 0.17 & & $\mathrm{mg} / \mathrm{Kg}$ & 10 & 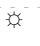 & 6020 & Total/NA \\
\hline Nickel & 51 & & 0.17 & & $\mathrm{mg} / \mathrm{Kg}$ & 10 & 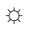 & 6020 & Total/NA \\
\hline Selenium & 2.5 & & 0.58 & & $\mathrm{mg} / \mathrm{Kg}$ & 10 & 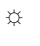 & 6020 & Total/NA \\
\hline Silver & 0.23 & & 0.17 & & $\mathrm{mg} / \mathrm{Kg}$ & 10 & 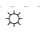 & 6020 & Total/NA \\
\hline Thallium & 0.34 & & 0.33 & & $\mathrm{mg} / \mathrm{Kg}$ & 10 & 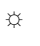 & 6020 & Total/NA \\
\hline Vanadium & 29 & & 0.42 & & $\mathrm{mg} / \mathrm{Kg}$ & 10 & 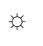 & 6020 & Total/NA \\
\hline Zinc & 140 & & 0.58 & & $\mathrm{mg} / \mathrm{Kg}$ & 10 & 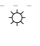 & 6020 & Total/NA \\
\hline Mercury & 0.98 & & 0.14 & & $\mathrm{mg} / \mathrm{Kg}$ & 10 & 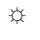 & $7471 \mathrm{~A}$ & Total/NA \\
\hline Chloride & 3400 & & 33 & & $\mathrm{mg} / \mathrm{Kg}$ & 10 & $x$ & 300.0 & Soluble \\
\hline Sulfate & 3300 & & 30 & & $\mathrm{mg} / \mathrm{Kg}$ & 10 & - & 300.0 & Soluble \\
\hline
\end{tabular}


Method: 6010B - Metals (ICP)

\section{Analyte}

\section{Aluminum}

Iron

Magnesium

Sodium

Method: 6020 - Metals (ICP/MS)

\section{Analyte}

Antimony

Arsenic

Barium

Beryllium

Cadmium

Chromium

Copper

Lead

Nickel

Selenium

Silver

Thallium

Vanadium

Zinc

Result
$\begin{array}{r}15000 \\ 43000\end{array}$
9000
ND

$\mathbf{R L}$
2800
940
5200
9400

\begin{tabular}{rr} 
Result & Qualifier \\
\cline { 2 - 2 } 0.21 & \\
15 & \\
550 & \\
0.95 & \\
0.44 & \\
54 & \\
47 & \\
150 \\
50 \\
0.67 \\
$N D$ \\
$N D$ \\
38 \\
120
\end{tabular}

\begin{tabular}{r}
$\mathbf{R L}$ \\
\hline 0.17 \\
0.43 \\
0.17 \\
0.17 \\
0.17 \\
0.17 \\
0.17 \\
0.17 \\
0.17 \\
0.60 \\
0.17 \\
0.34 \\
0.43 \\
0.60
\end{tabular}

$\frac{\mathrm{RL}}{0.17}$

MDL Unit

$\mathrm{mg} / \mathrm{Kg}$

$\mathrm{mg} / \mathrm{Kg}$

$\mathrm{mg} / \mathrm{Kg}$

$\mathrm{mg} / \mathrm{Kg}$

$\mathrm{mg} / \mathrm{Kg}$

$\mathrm{mg} / \mathrm{Kg}$

$\mathrm{mg} / \mathrm{Kg}$

$\mathrm{mg} / \mathrm{Kg}$

$\mathrm{mg} / \mathrm{Kg}$

$\mathrm{mg} / \mathrm{Kg}$

$\mathrm{mg} / \mathrm{Kg}$

$\mathrm{mg} / \mathrm{Kg}$

$\mathrm{mg} / \mathrm{Kg}$

$\mathrm{mg} / \mathrm{Kg}$

\begin{tabular}{|c|c|}
\hline Analyzed & Dil Fac \\
\hline 03/01/11 08:04 & 100 \\
\hline 03/01/11 08:04 & 100 \\
\hline 03/01/11 08:04 & 100 \\
\hline 03/01/11 08:04 & 100 \\
\hline
\end{tabular}

Analyzed

03/03/11 16:24

03/03/11 16:24

03/03/11 16:24

$03 / 03 / 1116: 24$

03/03/11 16:24

$03 / 03 / 1116: 24$

$03 / 03 / 1116: 24$

03/03/11 16:24

03/03/11 16:24

03/03/11 16:24

03/03/11 16:24

03/03/11 16:24

$03 / 03 / 1116: 24$

$03 / 03 / 1116: 24$

Dil Fac

10

10

\section{0}

10

$$
10
$$$$
10
$$$$
10
$$$$
10
$$$$
10
$$$$
10
$$$$
10
$$$$
10
$$$$
10
$$

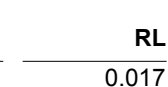

$\frac{\mathbf{R L}}{017}$

$\underline{M D L} \frac{\text { Unit }}{\mathrm{mg} / \mathrm{Kg}}$

Method: 7471A - Mercury (CVAA)

Analyte

Mercury 0.77

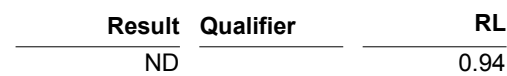

RL

RL Unit $\frac{\text { U }}{\mathrm{mg} / \mathrm{Kg}}$

$\frac{\text { D }}{3} \frac{\text { Prepared }}{02 / 24 / 1117: 04}$

$\frac{\text { Analyzed }}{02 / 28 / 1114: 39}$

Dil Fac

General Chemistry - Soluble

\begin{tabular}{|c|c|c|c|c|c|c|c|c|c|}
\hline Analyte & Result & Qualifier & RL & MDL & Unit & D & Prepared & Analyzed & Dil Fac \\
\hline Chloride & 390 & & 3.3 & & $\mathrm{mg} / \mathrm{Kg}$ & 要 & & 03/08/11 09:49 & 1 \\
\hline Sulfate & 210 & & 3.0 & & $\mathrm{mg} / \mathrm{Kg}$ & 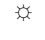 & & 03/08/11 09:49 & 1 \\
\hline
\end{tabular}

\section{Client Sample ID: Brontosaurus \#1 \#2- intermed hole}

Lab Sample ID: AUB0026-02

Date Collected: 02/17/11 10:30

Matrix: Soil

Date Received: 02/18/11 13:50

Percent Solids: 99.3

Sampler Name:

Sampler Phone Number: (907) 272-1232
Method: 6010B - Metals (ICP)

Analyte

Aluminum

Iron

Magnesium

Sodium

Method: 6020 - Metals (ICP/MS)

Analyte

Antimony

Arsenic

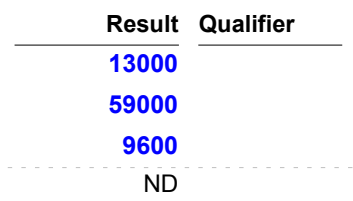

\begin{tabular}{r}
$\mathbf{R L}$ \\
\hline 2000 \\
660 \\
3600 \\
6600
\end{tabular}

\begin{tabular}{|c|c|}
\hline MDL & Unit \\
\hline & $\mathrm{mg} / \mathrm{Kg}$ \\
\hline & $\mathrm{mg} / \mathrm{Kg}$ \\
\hline & $\mathrm{mg} / \mathrm{Kg}$ \\
\hline & $\mathrm{mg} / \mathrm{Kg}$ \\
\hline
\end{tabular}

$\mathrm{mg} / \mathrm{Kg}$

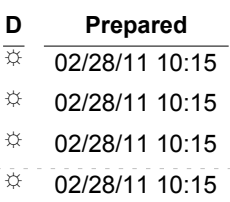

\begin{tabular}{|c|c|}
\hline Analyzed & Dil Fac \\
\hline 03/01/11 08:09 & 100 \\
\hline 03/01/11 08:09 & 100 \\
\hline 03/01/11 08:09 & 100 \\
\hline 03/01/11 08:09 & 100 \\
\hline
\end{tabular}

$\mathbf{R L}$

0.46

MDL Unit

$\mathrm{mg} / \mathrm{Kg}$

D

\begin{tabular}{l} 
Prepared \\
\hline $03 / 02 / 1110: 22$ \\
$03 / 02 / 1110: 22$
\end{tabular}

\begin{tabular}{|c|c|}
\hline Analyzed & Dil Fac \\
\hline $03 / 03 / 11$ 16:36 & 10 \\
\hline 03/03/11 16:36 & 10 \\
\hline
\end{tabular}


Method: 6020 - Metals (ICP/MS) (Continued)

\begin{tabular}{|c|c|c|c|c|c|c|c|c|c|}
\hline Analyte & Result & Qualifier & $\mathbf{R L}$ & MDL & Unit & D & Prepared & Analyzed & Dil Fac \\
\hline Barium & 240 & & 0.18 & & $\mathrm{mg} / \mathrm{Kg}$ & $\bar{s}$ & $03 / 02 / 11$ 10:22 & 03/03/11 16:36 & 10 \\
\hline Beryllium & 0.90 & & 0.18 & & $\mathrm{mg} / \mathrm{Kg}$ & 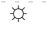 & $03 / 02 / 11 \quad 10: 22$ & $03 / 03 / 1116: 36$ & 10 \\
\hline Cadmium & 0.26 & & 0.18 & & $\mathrm{mg} / \mathrm{Kg}$ & 家 & 03/02/11 10:22 & 03/03/11 16:36 & 10 \\
\hline Chromium & 36 & & 0.18 & & $\mathrm{mg} / \mathrm{Kg}$ & 家 & 03/02/11 10:22 & 03/03/11 16:36 & 10 \\
\hline Copper & 51 & & 0.18 & & $\mathrm{mg} / \mathrm{Kg}$ & 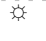 & $03 / 02 / 11 \quad 10: 22$ & $03 / 03 / 1116: 36$ & 10 \\
\hline Lead & 34 & & 0.18 & & $\mathrm{mg} / \mathrm{Kg}$ & 凉 & 03/02/11 10:22 & 03/03/11 16:36 & 10 \\
\hline Nickel & 52 & & 0.18 & & $\mathrm{mg} / \mathrm{Kg}$ & 家 & $03 / 02 / 11$ 10:22 & 03/03/11 16:36 & 10 \\
\hline Selenium & 0.70 & & 0.64 & & $\mathrm{mg} / \mathrm{Kg}$ & 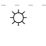 & $03 / 02 / 1110: 22$ & $03 / 03 / 1116: 36$ & 10 \\
\hline Silver & ND & & 0.18 & & $\mathrm{mg} / \mathrm{Kg}$ & 文 & $03 / 02 / 11$ 10:22 & 03/03/11 16:36 & 10 \\
\hline Thallium & ND & & 0.36 & & $\mathrm{mg} / \mathrm{Kg}$ & ; & 03/02/11 10:22 & 03/03/11 16:36 & 10 \\
\hline Vanadium & 36 & & 0.46 & & $\mathrm{mg} / \mathrm{Kg}$ & 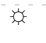 & $03 / 02 / 11 \quad 10: 22$ & $03 / 03 / 1116: 36$ & 10 \\
\hline Zinc & 110 & & 0.64 & & $\mathrm{mg} / \mathrm{Kg}$ & 家 & 03/02/11 10:22 & 03/03/11 16:36 & 10 \\
\hline
\end{tabular}

Method: 7471A - Mercury (CVAA)

Analyte

Mercury

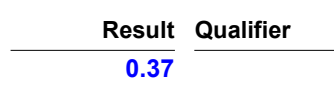

$\frac{\mathbf{R L}}{0.013}$

RL Unit

$\frac{\text { D }}{03 / 03 / 1109: 03} \frac{\text { Prepared }}{03 / 03 / 1112: 07}$

Dil Fac

General Chemistry

Analyte

Result Qualifier

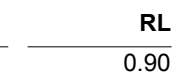

$\frac{\mathbf{R L}}{0.90}$

RL Unit

$\frac{\text { D }}{02 / 24 / 1117: 04}$

$\frac{\text { Analyzed }}{02 / 28 / 1114: 39}$

Dil Fac

General Chemistry - Soluble

\begin{tabular}{|c|c|c|c|c|c|c|c|c|c|}
\hline Analyte & Result & Qualifier & RL & MDL & Unit & D & Prepared & Analyzed & Dil Fac \\
\hline Chloride & 75 & & 3.3 & & $\mathrm{mg} / \mathrm{Kg}$ & $\bar{x}$ & & $03 / 08 / 1110: 38$ & 1 \\
\hline Sulfate & 750 & & 30 & & $\mathrm{mg} / \mathrm{Kg}$ & 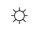 & & 03/08/11 15:52 & 10 \\
\hline
\end{tabular}

Client Sample ID: Brontosaurus \#1 \#3- intermed hole

Lab Sample ID: AUB0026-03

Date Collected: 02/17/11 11:00

Matrix: Soil

Date Received: 02/18/11 13:50

Percent Solids: 99.1

Sampler Name:

Sampler Phone Number: (907) 272-1232

Method: 6010B - Metals (ICP)

\section{Analyte}

Aluminum

Iron

Magnesium

Sodium

$\begin{array}{rr}\text { Result } & \text { Qualifier } \\ & \\ 40000 \\ 8000 \\ \text { ND }\end{array}$

Method: 6020 - Metals (ICP/MS)

\section{Analyte}

Antimony

Arsenic

Barium

Beryllium

Cadmium

Chromium

Copper

Lead

Nickel

Selenium

$\begin{array}{rr}\text { Result } & \text { Qualifier } \\ & \\ 21 & \\ 150 \\ 0.86 \\ 0.98 \\ 27 \\ 47 \\ 74 \\ 53 \\ 3.8\end{array}$

D

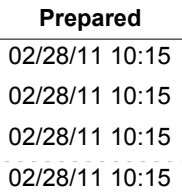

$\mathrm{mg} / \mathrm{Kg}$

\begin{tabular}{l} 
Unit \\
\hline $\mathrm{mg} / \mathrm{Kg}$ \\
$\mathrm{mg} / \mathrm{Kg}$ \\
$\mathrm{mg} / \mathrm{Kg}$ \\
$\mathrm{mg} / \mathrm{Kg}$
\end{tabular}

02/28/11 10:15

\begin{tabular}{|c|c|}
\hline Analyzed & Dil Fac \\
\hline $03 / 01 / 11$ 08:14 & 100 \\
\hline 03/01/11 08:14 & 100 \\
\hline 03/01/11 08:14 & 100 \\
\hline $03 / 01 / 1108: 14$ & 100 \\
\hline
\end{tabular}

DL Unit \begin{tabular}{l}
\hline $\mathrm{mg} / \mathrm{Kg}$ \\
$\mathrm{mg} / \mathrm{Kg}$ \\
$\mathrm{mg} / \mathrm{Kg}$ \\
$\mathrm{mg} / \mathrm{Kg}$ \\
$\mathrm{mg} / \mathrm{Kg}$ \\
$\mathrm{mg} / \mathrm{Kg}$ \\
$\mathrm{mg} / \mathrm{Kg}$ \\
$\mathrm{mg} / \mathrm{Kg}$ \\
$\mathrm{mg} / \mathrm{Kg}$ \\
$\mathrm{mg} / \mathrm{Kg}$
\end{tabular}

$$
\text { D }
$$

\begin{tabular}{|c|c|}
\hline Analyzed & Dil Fac \\
\hline 03/03/11 17:00 & 10 \\
\hline 03/03/11 17:00 & 10 \\
\hline 03/03/11 17:00 & 10 \\
\hline $03 / 03 / 1117: 00$ & 10 \\
\hline 03/03/11 17:00 & 10 \\
\hline 03/03/11 17:00 & 10 \\
\hline $03 / 03 / 1117: 00$ & 10 \\
\hline 03/03/11 17:00 & 10 \\
\hline 03/03/11 17:00 & 10 \\
\hline $03 / 03 / 1117: 00$ & 10 \\
\hline
\end{tabular}

0.15

0.15

0.15

0.15

0.53 
Method: 6020 - Metals (ICP/MS) (Continued)

\begin{tabular}{|c|c|c|c|c|c|c|c|c|c|}
\hline Analyte & Result & Qualifier & RL & MDL & Unit & D & Prepared & Analyzed & Dil Fac \\
\hline Silver & 0.29 & & 0.15 & & $\mathrm{mg} / \mathrm{Kg}$ & $\bar{x}$ & $03 / 02 / 11$ 10:22 & 03/03/11 17:00 & 10 \\
\hline Thallium & 0.33 & & 0.30 & & $\mathrm{mg} / \mathrm{Kg}$ & 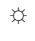 & 03/02/11 10:22 & 03/03/11 17:00 & 10 \\
\hline Vanadium & 40 & & 0.38 & & $\mathrm{mg} / \mathrm{Kg}$ & 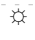 & $03 / 02 / 1110: 22$ & $03 / 03 / 1117: 00$ & 10 \\
\hline Zinc & 140 & & 0.53 & & $\mathrm{mg} / \mathrm{Kg}$ & 弶 & 03/02/11 10:22 & 03/03/11 17:00 & 10 \\
\hline
\end{tabular}

Method: 7471A - Mercury (CVAA)

\section{Analyte}

Mercury
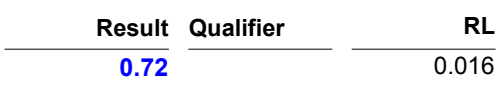

$\mathbf{R L}$

MDL Unit

$\frac{\mathrm{D}}{3 \times}$

Prepared

Analyzed

Dil Fac

General Chemistry

Analyte

Result Qualifier

$\frac{\mathbf{R L}}{0.84}$

RL Unit

$\frac{\text { D }}{02 / 24 / 1117: 04}$

$\frac{\text { Analyzed }}{02 / 28 / 1114: 39}$

Dil Fac

Cyanide, Total

$\mathrm{ND}$

$\mathrm{mg} / \mathrm{Kg}$

$\frac{\text { D }}{3} \quad$ Prepared

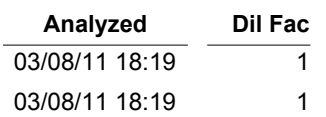

Lab Sample ID: AUB0026-04

Matrix: Soil

Percent Solids: 99.1

Sampler Phone Number: (907) 272-1232

\section{Client Sample ID: Walakpa \#1 \#1- surface hole}

Date Collected: 02/17/11 11:30

Date Received: 02/18/11 13:50

Sampler Name:
Method: 6010B - Metals (ICP)

\section{Analyte}

Aluminum

Iron

Magnesium

Sodium

Method: 6020 - Metals (ICP/MS)

\section{Analyte}

Antimony

Arsenic

Barium

Beryllium

Cadmium

Chromium

Copper

Lead

Nickel

Selenium

Silver

Thallium

Vanadium

Zinc

Result Qualifier
14000
40000
10000
ND

\begin{tabular}{r}
$\mathbf{R L}$ \\
\hline 2600 \\
860 \\
4700 \\
8600
\end{tabular}

RL

860

8600
MDL Unit

$$
\begin{aligned}
& \mathrm{mg} / \mathrm{Kg} \\
& \mathrm{mg} / \mathrm{Kg} \\
& \mathrm{mg} / \mathrm{Kg} \\
& \mathrm{mg} / \mathrm{Kg}
\end{aligned}
$$

\begin{tabular}{|c|c|c|}
\hline RL & MDL & Unit \\
\hline 0.16 & & $\mathrm{mg} / \mathrm{Kg}$ \\
\hline 0.41 & & $\mathrm{mg} / \mathrm{Kg}$ \\
\hline 0.16 & & $\mathrm{mg} / \mathrm{Kg}$ \\
\hline 0.16 & & $\mathrm{mg} / \mathrm{Kg}$ \\
\hline 0.16 & & $\mathrm{mg} / \mathrm{Kg}$ \\
\hline 0.16 & & $\mathrm{mg} / \mathrm{Kg}$ \\
\hline 0.16 & & $\mathrm{mg} / \mathrm{Kg}$ \\
\hline 0.16 & & $\mathrm{mg} / \mathrm{Kg}$ \\
\hline 0.16 & & $\mathrm{mg} / \mathrm{Kg}$ \\
\hline 0.57 & & $\mathrm{mg} / \mathrm{Kg}$ \\
\hline 0.16 & & $\mathrm{mg} / \mathrm{Kg}$ \\
\hline 0.33 & & $\mathrm{mg} / \mathrm{Kg}$ \\
\hline 0.41 & & $\mathrm{mg} / \mathrm{Kg}$ \\
\hline 0.57 & & $\mathrm{mg} / \mathrm{Kg}$ \\
\hline
\end{tabular}

\begin{tabular}{|c|c|}
\hline Analyzed & Dil Fac \\
\hline 03/01/11 08:19 & 100 \\
\hline 03/01/11 08:19 & 100 \\
\hline 03/01/11 08:19 & 100 \\
\hline 03/01/11 08:19 & 100 \\
\hline
\end{tabular}

\begin{tabular}{|c|}
\hline \\
\hline $03 / 02 / 11$ 10:2 \\
\hline 03/02/11 10:22 \\
\hline 03/02/11 10:22 \\
\hline 03/02/11 10:22 \\
\hline 03/02/11 10:22 \\
\hline 03/02/11 10:22 \\
\hline 03/02/11 10:22 \\
\hline 03/02/11 10:22 \\
\hline 03/02/11 10:22 \\
\hline $03 / 02 / 1110: 22$ \\
\hline 03/02/11 10:22 \\
\hline 03/02/11 10:22 \\
\hline 03/02/11 10:22 \\
\hline 03/02/11 10:2 \\
\hline
\end{tabular}

$\mathrm{mg} / \mathrm{Kg}$

\begin{tabular}{|c|c|}
\hline Analyzed & Dil Fac \\
\hline 03/03/11 17:12 & 10 \\
\hline 03/03/11 17:12 & 10 \\
\hline 03/03/11 17:12 & 10 \\
\hline $03 / 03 / 1117: 12$ & 10 \\
\hline 03/03/11 17:12 & 10 \\
\hline 03/03/11 17:12 & 10 \\
\hline $03 / 03 / 11 \quad 17: 12$ & 10 \\
\hline 03/03/11 17:12 & 10 \\
\hline 03/03/11 17:12 & 10 \\
\hline $03 / 03 / 1117: 12$ & 10 \\
\hline 03/03/11 17:12 & 10 \\
\hline 03/03/11 17:12 & 10 \\
\hline $03 / 03 / 1117: 12$ & 10 \\
\hline 03/03/11 17:12 & 10 \\
\hline
\end{tabular}

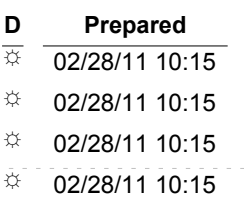

Method: 7471A - Mercury (CVAA) Analyte 


\section{General Chemistry}

\begin{tabular}{|c|c|c|c|c|c|c|c|c|c|}
\hline Analyte & Result & Qualifier & RL & RL & Unit & D & Prepared & Analyzed & Dil Fac \\
\hline Cyanide, Total & ND & & 0.97 & & $\mathrm{mg} / \mathrm{Kg}$ & $\bar{x}$ & 02/24/11 17:04 & 02/28/11 14:39 & 1 \\
\hline \multicolumn{10}{|c|}{ General Chemistry - Soluble } \\
\hline Analyte & Result & Qualifier & RL & MDL & Unit & D & Prepared & Analyzed & Dil Fac \\
\hline Chloride & 460 & & 3.3 & & $\mathrm{mg} / \mathrm{Kg}$ & 豙 & & 03/08/11 12:00 & 1 \\
\hline Sulfate & 1400 & & 30 & & $\mathrm{mg} / \mathrm{Kg}$ & 弶 & & 03/08/11 16:25 & 10 \\
\hline
\end{tabular}

Client Sample ID: Walakpa \#1 \#2- intermed. hole

Lab Sample ID: AUB0026-05

Date Collected: 02/17/11 12:00

Matrix: Soil

Date Received: 02/18/11 13:50

Percent Solids: 99.2

Sampler Name: Sampler Phone Number: (907) 272-1232
Method: 6010B - Metals (ICP)

\section{Analyte}

\section{Aluminum}

Iron

Magnesium

Sodium

Method: 6020 - Metals (ICP/MS)

\section{Analyte}

Antimony

Arsenic

Barium

Beryllium

Cadmium

Chromium

Copper

Lead

Nickel

Selenium

Silver

Thallium

Vanadium

Zinc

Result
$\begin{array}{r}14000 \\ 47000 \\ 9600\end{array}$
ND

$\begin{array}{rr}\text { Result } & \text { Qualifier } \\ & \\ 12 & \\ 170 & \\ 0.91 \\ 0.31 \\ 30 \\ 36 \\ 130 \\ 46 \\ 0.74 \\ N D \\ N D \\ 34 \\ 340\end{array}$

\begin{tabular}{rll}
$\mathbf{R L}$ & MDL & Unit \\
\cline { 1 - 1 } 2100 & $\mathrm{mg} / \mathrm{Kg}$ \\
700 & $\mathrm{mg} / \mathrm{Kg}$ \\
3900 & $\mathrm{mg} / \mathrm{Kg}$ \\
7000 & $\mathrm{mg} / \mathrm{Kg}$
\end{tabular}

$\frac{\mathbf{D}}{\mathrm{s}}$

\begin{tabular}{lll} 
D & Prepared \\
\cline { 2 - 2 } & $02 / 28 / 11$ & $10: 15$ \\
$02 / 28 / 11$ & $10: 15$ \\
$02 / 28 / 11$ & $10: 15$ \\
$02 / 28 / 11$ & $10: 15$
\end{tabular}
MDL Unit

\section{$\mathrm{mg} / \mathrm{Kg}$}

$\mathrm{mg} / \mathrm{Kg}$

$\mathrm{mg} / \mathrm{Kg}$

$\mathrm{mg} / \mathrm{Kg}$

$\mathrm{mg} / \mathrm{Kg}$

$\mathrm{mg} / \mathrm{Kg}$

$\mathrm{mg} / \mathrm{Kg}$

$\mathrm{mg} / \mathrm{Kg}$

$\mathrm{mg} / \mathrm{Kg}$

$\mathrm{mg} / \mathrm{Kg}$

$\mathrm{mg} / \mathrm{Kg}$

$\mathrm{mg} / \mathrm{Kg}$

$\mathrm{mg} / \mathrm{Kg}$

$\mathrm{mg} / \mathrm{Kg}$

Method: 7471A - Mercury (CVAA)

Analyte

Mercury
Result Qualifier

$\frac{\mathbf{R L}}{0.12}$

$\stackrel{\text { MDL }}{\frac{\text { Unit }}{\mathrm{mg} / \mathrm{Kg}}}$

$\frac{\text { Prepared }}{03 / 03 / 1109 \cdot 03}$

$\frac{\text { Analyzed }}{03 / 03 / 1112: 45} \frac{\text { Dil Fac }}{10}$

General Chemistry

Analyte

Cyanide, Total

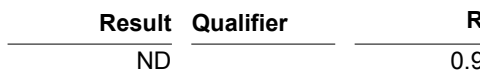

$\frac{\mathbf{R L}}{0.91}$

RL Unit

$\frac{\text { D }}{3} \frac{\text { Prepared }}{02 / 24 / 1117 \cdot 04}$

$\frac{\text { Analyzed }}{02 / 28 / 1114: 39} \frac{\text { Dil Fac }}{1}$

General Chemistry - Soluble

Analyte

Chloride

Result

410

\begin{tabular}{r}
$\mathbf{R L}$ \\
\hline 3.3 \\
3.0
\end{tabular}

$\frac{\text { MDL }}{\frac{\mathrm{mg} / \mathrm{Kg}}{\mathrm{mg} / \mathrm{Kg}}} \frac{\mathrm{D}}{\mathrm{s}}$

$\frac{\text { D }}{\text { Prepared }}$

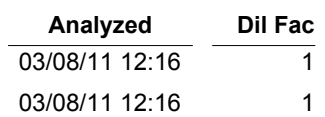


Method: 6010B - Metals (ICP)

\section{Analyte}

\section{Aluminum}

Iron

Magnesium

Sodium

Method: 6020 - Metals (ICP/MS)

\section{Analyte}

Antimony

Arsenic

Barium

Beryllium

Cadmium

Chromium

Copper

Lead

Nickel

Selenium

Silver

Thallium

Vanadium

Zinc

Method: 7471A - Mercury (CVAA)

Analyte

Mercury

General Chemistry

Analyte

Cyanide, Total

\begin{tabular}{|c|c|}
\hline Result & $\mathbf{Q}$ \\
\hline 12000 & \\
\hline 42000 & \\
\hline 7600 & \\
\hline ND & \\
\hline
\end{tabular}

\begin{tabular}{r}
$\mathbf{R L}$ \\
\hline 2900 \\
960 \\
5300 \\
9600
\end{tabular}

\begin{tabular}{rr} 
Result & Qualifier \\
\cline { 2 - 2 } 0.33 & \\
21 & \\
220 & \\
0.70 \\
0.58 \\
23 \\
47 \\
86 \\
48 \\
4.2 \\
0.29 \\
$N D$ \\
45 \\
150
\end{tabular}

\begin{tabular}{r}
$\mathbf{R L}$ \\
\hline 0.16 \\
0.39 \\
0.16 \\
0.16 \\
0.16 \\
0.16 \\
0.16 \\
0.16 \\
0.16 \\
0.55 \\
0.16 \\
0.32 \\
0.39 \\
0.55
\end{tabular}

$\begin{array}{ll}\text { MDL } & \text { Unit } \\ \mathrm{mg} / \mathrm{Kg} \\ \mathrm{mg} / \mathrm{Kg} \\ \mathrm{mg} / \mathrm{Kg} \\ \mathrm{mg} / \mathrm{Kg}\end{array}$

\begin{tabular}{lll} 
D & Prepared \\
\cline { 2 - 2 } & $02 / 28 / 11$ & $10: 15$ \\
$02 / 28 / 11$ & $10: 15$ \\
$02 / 28 / 11$ & $10: 15$ \\
$02 / 28 / 11$ & $10: 15$
\end{tabular}$$
\text { D }
$$

Prepared 03/02/11 10:22

03/02/11 10:22

03/02/11 10:22

03/02/11 10:22

03/02/11 10:22

03/02/11 10:22

03/02/11 10:22

03/02/11 10:22

03/02/11 10:22

03/02/11 10:22

03/02/11 10:22

03/02/11 10:22

03/02/11 10:22

03/02/11 10:22

\begin{tabular}{|c|c|}
\hline Analyzed & Dil Fac \\
\hline 03/01/11 08:29 & 100 \\
\hline 03/01/11 08:29 & 100 \\
\hline 03/01/11 08:29 & 100 \\
\hline 03/01/11 08:29 & 100 \\
\hline
\end{tabular}

Analyzed

Dil Fac

03/03/11 17:36

03/03/11 17:36

03/03/11 17:36

03/03/11 17:36

03/03/11 17:36

03/03/11 17:36

$03 / 03 / 1117: 36$

03/03/11 17:36

03/03/11 17:36

03/03/11 17:36

03/03/11 17:36

03/03/11 17:36

03/03/11 17:36

$03 / 03 / 1117: 36$

\begin{tabular}{|c|c|c|c|c|c|c|c|c|}
\hline Result & Qualifier & $\mathbf{R L}$ & MDL & Unit & $\underline{\mathrm{D}}$ & Prepared & Analyzed & Dil Fac \\
\hline 0.49 & & 0.015 & & $\mathrm{mg} / \mathrm{Kg}$ & $\bar{s}$ & 03/03/11 09:03 & $03 / 03 / 11$ 12:25 & 1 \\
\hline
\end{tabular}

General Chemistry - Soluble

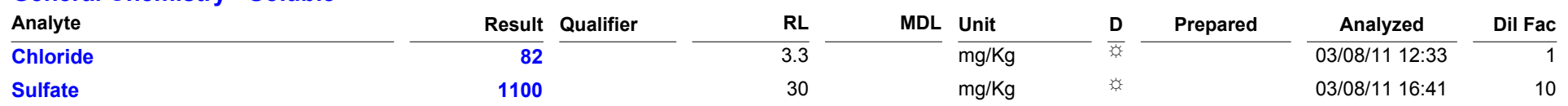

\section{Client Sample ID: S. Barrow 19 \#1- surface hole}

Lab Sample ID: AUB0026-07

Date Collected: 02/17/11 13:00

Matrix: Soil

Date Received: 02/18/11 13:50

Percent Solids: 99.1

Sampler Name:

Sampler Phone Number: (907) 272-1232

Method: 6010B - Metals (ICP)

Aluminum

Iron

Magnesium

Sodium

\section{Analyte}

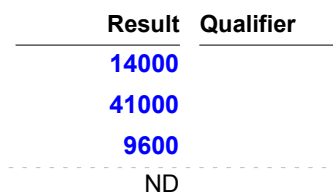

$\mathbf{R L}$
2500

840

4600

8400

\begin{tabular}{|c|c|}
\hline MDL & Unit \\
\hline & $\mathrm{mg} / \mathrm{Kg}$ \\
\hline & $\mathrm{mg} / \mathrm{Kg}$ \\
\hline & $\mathrm{mg} / \mathrm{Kg}$ \\
\hline & $\mathrm{mg} / \mathrm{Kg}$ \\
\hline
\end{tabular}

$\mathrm{mg} / \mathrm{Kg}$

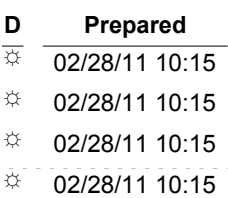

\begin{tabular}{|c|c|}
\hline Analyzed & Dil Fac \\
\hline 03/01/11 08:34 & 100 \\
\hline 03/01/11 08:34 & 100 \\
\hline 03/01/11 08:34 & 100 \\
\hline 03/01/11 08:34 & 100 \\
\hline
\end{tabular}

Method: 6020 - Metals (ICP/MS)

Analyte

Antimony

Result Qualifier

$\frac{\mathbf{R L}}{0.17}$

0.42

18

$\begin{array}{ll}\text { MDL } & \frac{\text { Unit }}{\mathrm{mg} / \mathrm{Kg}} \\ \mathrm{mg} / \mathrm{Kg}\end{array}$

D

\begin{tabular}{c} 
Prepared \\
\hline $3 / 02 / 1110: 22$
\end{tabular}

$03 / 02 / 1110: 22$

\begin{tabular}{|c|c|}
\hline Analyzed & Dil Fac \\
\hline $03 / 03 / 11 \quad 17: 48$ & 10 \\
\hline 03/03/11 17:48 & 10 \\
\hline
\end{tabular}


Client: Petrotechnical Resources of Alaska

Project/Site: [none]

Client Sample ID: S. Barrow 19 \#1- surface hole

Lab Sample ID: AUB0026-07

Date Collected: 02/17/11 13:00

Matrix: Soil

Date Received: 02/18/11 13:50

Percent Solids: 99.1

Sampler Name:

Sampler Phone Number: (907) 272-1232

Method: 6020 - Metals (ICP/MS) (Continued)

\begin{tabular}{|c|c|c|c|c|c|c|c|c|c|}
\hline Analyte & Result & Qualifier & RL & MDL & Unit & D & Prepared & Analyzed & Dil Fac \\
\hline Barium & 800 & & 0.17 & & $\mathrm{mg} / \mathrm{Kg}$ & $\bar{x}$ & $03 / 02 / 11$ 10:22 & 03/03/11 17:48 & 10 \\
\hline Beryllium & 0.98 & & 0.17 & & $\mathrm{mg} / \mathrm{Kg}$ & 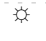 & $03 / 02 / 1110: 22$ & $03 / 03 / 1117: 48$ & 10 \\
\hline Cadmium & 0.32 & & 0.17 & & $\mathrm{mg} / \mathrm{Kg}$ & 家 & $03 / 02 / 1110: 22$ & 03/03/11 17:48 & 10 \\
\hline Chromium & 26 & & 0.17 & & $\mathrm{mg} / \mathrm{Kg}$ & 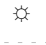 & $03 / 02 / 1110: 22$ & 03/03/11 17:48 & 10 \\
\hline Copper & 30 & & 0.17 & & $\mathrm{mg} / \mathrm{Kg}$ & 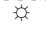 & 03/02/11 10:22 & $03 / 03 / 1117: 48$ & 10 \\
\hline Lead & 230 & & 0.17 & & $\mathrm{mg} / \mathrm{Kg}$ & 㔔 & 03/02/11 10:22 & 03/03/11 17:48 & 10 \\
\hline Nickel & 39 & & 0.17 & & $\mathrm{mg} / \mathrm{Kg}$ & 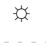 & 03/02/11 10:22 & 03/03/11 17:48 & 10 \\
\hline Selenium & 0.63 & & 0.59 & & $\mathrm{mg} / \mathrm{Kg}$ & 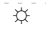 & $03 / 02 / 11 \quad 10: 22$ & $03 / 03 / 1117: 48$ & 10 \\
\hline Silver & ND & & 0.17 & & $\mathrm{mg} / \mathrm{Kg}$ & 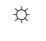 & $03 / 02 / 11$ 10:22 & 03/03/11 17:48 & 10 \\
\hline Thallium & ND & & 0.34 & & $\mathrm{mg} / \mathrm{Kg}$ & 3 & $03 / 02 / 11$ 10:22 & 03/03/11 17:48 & 10 \\
\hline Vanadium & 36 & & 0.42 & & $\mathrm{mg} / \mathrm{Kg}$ & 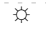 & $03 / 02 / 1110: 22$ & $03 / 03 / 1117: 48$ & 10 \\
\hline Zinc & 200 & & 0.59 & & $\mathrm{mg} / \mathrm{Kg}$ & 3 & 03/02/11 10:22 & 03/03/11 17:48 & 10 \\
\hline
\end{tabular}

Method: 7471A - Mercury (CVAA)

Analyte

Result Qualifier

$\frac{\mathbf{R L}}{0.14}$

MDL Unit

$\frac{\text { D }}{\text { Prepared }} \frac{\text { Analyzed }}{03 / 03 / 1109: 03} \frac{\text { Dil Fac }}{03 / 03 / 1112: 48} \frac{10}{10}$

Mercury

0.71

$\mathrm{mg} / \mathrm{Kg}$

General Chemistry

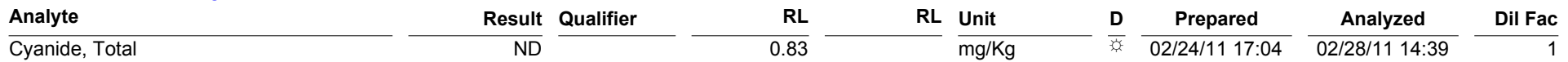

General Chemistry - Soluble

\begin{tabular}{|c|c|c|c|c|c|c|c|c|c|}
\hline Analyte & Result & Qualifier & RL & MDL & Unit & D & Prepared & Analyzed & Dil Fac \\
\hline Chloride & 750 & & 33 & & $\overline{\mathrm{mg} / \mathrm{Kg}}$ & $\bar{s}$ & & 03/08/11 16:57 & 10 \\
\hline Sulfate & 3300 & & 30 & & $\mathrm{mg} / \mathrm{Kg}$ & 㔔 & & 03/08/11 16:57 & 10 \\
\hline
\end{tabular}

Client Sample ID: S. Barrow 19 \#2- intermed. hole

Lab Sample ID: AUB0026-08

Date Collected: 02/17/11 13:30

Matrix: Soil

Date Received: 02/18/11 13:50

Percent Solids: $\mathbf{9 7 . 3}$

Sampler Name:

Sampler Phone Number: (907) 272-1232

Method: 6010B - Metals (ICP)

\section{Analyte}

Aluminum

Iron

Magnesium

Sodium

Method: 6020 - Metals (ICP/MS)

\section{Analyte}

Antimony

Arsenic

Barium

Beryllium

Cadmium

Chromium

Copper

Lead

Nickel

Selenium

$\begin{aligned} \text { Result } & \text { Qualifier } \\ & \\ 45000 & \\ 8300 & \\ \text { ND } & \end{aligned}$

$\mathbf{R L}$
2500
830
4600
8300

RL

830

600

8300

$\begin{array}{rr}\text { Result } & \text { Qualifier } \\ & \\ 36 & \\ 140 \\ 0.63 \\ 0.38 \\ 120 \\ 44 \\ 2400 \\ 53 \\ 3.2\end{array}$

\begin{tabular}{|c|c|c|}
\hline RL & MDL & Unit \\
\hline 0.19 & & $\mathrm{mg} / \mathrm{Kg}$ \\
\hline 0.48 & & $\mathrm{mg} / \mathrm{Kg}$ \\
\hline 0.19 & & $\mathrm{mg} / \mathrm{Kg}$ \\
\hline 0.19 & & $\mathrm{mg} / \mathrm{Kg}$ \\
\hline 0.19 & & $\mathrm{mg} / \mathrm{Kg}$ \\
\hline 0.19 & & $\mathrm{mg} / \mathrm{Kg}$ \\
\hline 0.19 & & $\mathrm{mg} / \mathrm{Kg}$ \\
\hline 19 & & $\mathrm{mg} / \mathrm{Kg}$ \\
\hline 0.19 & & $\mathrm{mg} / \mathrm{Kg}$ \\
\hline 0.68 & & $\mathrm{mg} / \mathrm{Kg}$ \\
\hline
\end{tabular}

\begin{tabular}{cc} 
D & Prepared \\
\hline $03 / 02 / 11$ & $10: 22$ \\
$03 / 02 / 11$ & $10: 22$ \\
$03 / 02 / 11$ & $10: 22$ \\
$03 / 02 / 11$ & $10: 22$ \\
$03 / 02 / 11$ & $10: 22$ \\
$03 / 02 / 11$ & $10: 22$ \\
\hline $03 / 02 / 1110: 22$ \\
$03 / 02 / 11$ & $10: 22$ \\
$03 / 02 / 11$ & $10: 22$ \\
\hline $03 / 02 / 11$ & $10: 22$
\end{tabular}

\begin{tabular}{|c|c|}
\hline Analyzed & Dil Fac \\
\hline 03/04/11 07:34 & 10 \\
\hline 03/04/11 07:34 & 10 \\
\hline 03/04/11 07:34 & 10 \\
\hline $03 / 04 / 11 \quad 07: 34$ & 10 \\
\hline 03/04/11 07:34 & 10 \\
\hline 03/04/11 07:34 & 10 \\
\hline 03/04/11 07:34 & 10 \\
\hline 03/04/11 07:55 & 1000 \\
\hline 03/04/11 07:34 & 10 \\
\hline 03/04/11 07:34 & 10 \\
\hline
\end{tabular}


Method: 6020 - Metals (ICP/MS) (Continued)

\begin{tabular}{|c|c|c|c|c|c|c|c|c|c|}
\hline Analyte & Result & Qualifier & RL & MDL & Unit & D & Prepared & Analyzed & Dil Fac \\
\hline Silver & 0.37 & & 0.19 & & $\mathrm{mg} / \mathrm{Kg}$ & 要 & $03 / 02 / 11$ 10:22 & 03/04/11 07:34 & 10 \\
\hline Thallium & ND & & 0.39 & & $\mathrm{mg} / \mathrm{Kg}$ & 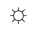 & 03/02/11 10:22 & 03/04/11 07:34 & 10 \\
\hline Vanadium & 46 & & 0.48 & & $\mathrm{mg} / \mathrm{Kg}$ & 策 & $03 / 02 / 1110: 22$ & $03 / 04 / 1107: 34$ & 10 \\
\hline Zinc & 640 & & 0.68 & & $\mathrm{mg} / \mathrm{Kg}$ & 弶 & 03/02/11 10:22 & 03/04/11 07:34 & 10 \\
\hline
\end{tabular}

Method: 7471A - Mercury (CVAA)

\section{Analyte}

Mercury
Result Qualifier

0.92

Result Qualifier

ND $\frac{\text { RL }}{0.90}$
$\frac{\mathrm{RL}}{0.16}$

MDL Unit

D

Prepared

Analyzed

Dil Fac

General Chemistry

Analyte

Cyanide, Total
RL Unit $\mathrm{mg} / \mathrm{Kg}$
$\frac{\mathrm{D}}{3}$

$\frac{\text { Prepared }}{02 / 24 / 1117: 04}$

Analyzed 02/28/11 14:39
RL

$\mathbf{R L}$
170
150

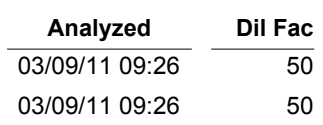

General Chemistry - Soluble

\begin{tabular}{|c|c|c|c|c|c|c|c|c|c|}
\hline Analyte & Result & Qualifier & RL & MDL & Unit & D & Prepared & Analyzed & Dil Fac \\
\hline Chloride & 11000 & & 170 & & $\overline{\mathrm{mg} / \mathrm{Kg}}$ & 离 & & 03/09/11 09:26 & 50 \\
\hline Sulfate & 11000 & & 150 & & $\mathrm{mg} / \mathrm{Kg}$ & 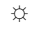 & & 03/09/11 09:26 & 50 \\
\hline
\end{tabular}

Client Sample ID: S. Barrow 19 \#3- intermed. hole

Lab Sample ID: AUB0026-09

Date Collected: 02/17/11 14:00

Matrix: Soil

Date Received: 02/18/11 13:50

Percent Solids: 98.0

Sampler Name:

Sampler Phone Number: (907) 272-1232

Method: 6010B - Metals (ICP)

\section{Analyte}

Aluminum

Iron

Magnesium

Sodium

Method: 6020 - Metals (ICP/MS)

\section{Analyte}

Antimony

Arsenic

Barium

Beryllium

Cadmium

Chromium

Copper

Lead

Nickel

Selenium

Silver

Thallium

Vanadium

Zinc

\begin{aligned} Result & Qualifier \\ \hline 8500 & \\ 44000 & \\ 5600 & \\ ND & \end{aligned}

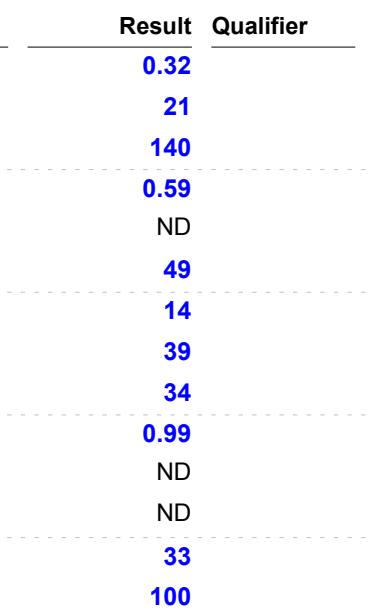

$\begin{array}{rll}\mathbf{R L} & \text { MDL } & \text { Unit } \\ \mathrm{mg} / \mathrm{Kg} \\ 830 & \mathrm{mg} / \mathrm{Kg} \\ 4600 & \mathrm{mg} / \mathrm{Kg} \\ 8300 & \mathrm{mg} / \mathrm{Kg}\end{array}$

$\frac{\mathbf{D}}{3-2}$ 02/28/11 10:15

$02 / 28 / 1110: 15$

\begin{tabular}{r}
$\mathbf{R L}$ \\
\hline 0.17 \\
0.42 \\
0.17 \\
0.17 \\
0.17 \\
0.17 \\
0.17 \\
0.17 \\
0.17 \\
0.59 \\
0.17 \\
0.34 \\
0.42 \\
0.59
\end{tabular}

\begin{tabular}{l} 
MDL Unit \\
\cline { 2 - 2 } $\mathrm{mg} / \mathrm{Kg}$ \\
$\mathrm{mg} / \mathrm{Kg}$ \\
$\mathrm{mg} / \mathrm{Kg}$ \\
$\mathrm{mg} / \mathrm{Kg}$ \\
$\mathrm{mg} / \mathrm{Kg}$ \\
$\mathrm{mg} / \mathrm{Kg}$ \\
$\mathrm{mg} / \mathrm{Kg}$ \\
$\mathrm{mg} / \mathrm{Kg}$ \\
$\mathrm{mg} / \mathrm{Kg}$ \\
$\mathrm{mg} / \mathrm{Kg}$ \\
$\mathrm{mg} / \mathrm{Kg}$ \\
$\mathrm{mg} / \mathrm{Kg}$ \\
$\mathrm{mg} / \mathrm{Kg}$ \\
$\mathrm{mg} / \mathrm{Kg}$
\end{tabular}
$\frac{\text { Prepared }}{02 / 28 / 1110: 15}$ $02 / 28 / 1110: 15$

\begin{tabular}{|c|c|}
\hline Analyzed & Dil Fac \\
\hline $03 / 01 / 11$ 08:53 & 100 \\
\hline 03/01/11 08:53 & \\
\hline 03/01/11 08:53 & \\
\hline 03/01/11 08:53 & \\
\hline
\end{tabular}

\begin{tabular}{c} 
Prepared \\
\hline 03/02/11 10:22 \\
03/02/11 10:22 \\
03/02/11 10:22 \\
\hline 03/02/11 10:22 \\
03/02/11 10:22 \\
03/02/11 10:22 \\
\hline 03/02/11 10:22 \\
03/02/11 10:22 \\
03/02/11 10:22 \\
\hline 03/02/11 10:22 \\
03/02/11 10:22 \\
03/02/11 10:22 \\
\hline 03/02/11 10:22 \\
03/02/11 10:22
\end{tabular}

\begin{tabular}{|c|c|}
\hline Analyzed & Dil Fac \\
\hline 03/04/11 07:38 & 10 \\
\hline 03/04/11 07:38 & 10 \\
\hline 03/04/11 07:38 & 10 \\
\hline $03 / 04 / 1107: 38$ & 10 \\
\hline 03/04/11 07:38 & 10 \\
\hline 03/04/11 07:38 & 10 \\
\hline $03 / 04 / 1107: 38$ & 10 \\
\hline 03/04/11 07:38 & 10 \\
\hline 03/04/11 07:38 & 10 \\
\hline 03/04/11 07:38 & 10 \\
\hline 03/04/11 07:38 & 10 \\
\hline 03/04/11 07:38 & 10 \\
\hline $03 / 04 / 1107: 38$ & 10 \\
\hline 03/04/11 07:38 & 10 \\
\hline
\end{tabular}

Method: 7471A - Mercury (CVAA) Analyte

Mercury

Result Qualifier 0.65
$\frac{\mathbf{R L}}{0.013}$

MDL Unit
$\frac{D}{3}$ 


\section{General Chemistry}

\begin{tabular}{|c|c|c|c|c|c|c|c|c|c|}
\hline Analyte & Result & Qualifier & RL & $\mathbf{R L}$ & Unit & D & Prepared & Analyzed & Dil Fac \\
\hline Cyanide, Total & $\mathrm{ND}$ & & 0.82 & & $\mathrm{mg} / \mathrm{Kg}$ & $\bar{x}$ & 02/24/11 17:04 & $02 / 28 / 1114: 39$ & $\overline{1}$ \\
\hline \multicolumn{10}{|c|}{ General Chemistry - Soluble } \\
\hline Analyte & Result & Qualifier & $\mathbf{R L}$ & MDL & Unit & D & Prepared & Analyzed & Dil Fac \\
\hline Chloride & 12000 & & 330 & & $\mathrm{mg} / \mathrm{Kg}$ & 票 & & $03 / 08 / 1117: 30$ & 100 \\
\hline Sulfate & 5800 & & 300 & & $\mathrm{mg} / \mathrm{Kg}$ & 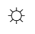 & & 03/08/11 17:30 & 100 \\
\hline
\end{tabular}

Client Sample ID: S. Barrow 14 \#1- surface hole

Date Collected: 02/17/11 14:30

Date Received: 02/18/11 13:50

Sampler Name:
Lab Sample ID: AUB0026-10

Matrix: Soil

Percent Solids: 99.2 Sampler Phone Number: (907) 272-1232
Method: 6010B - Metals (ICP)

\section{Analyte}

Aluminum

Iron

Magnesium

Sodium

Method: 6020 - Metals (ICP/MS)

\section{Analyte}

Antimony

Arsenic

Barium

Beryllium

Cadmium

Chromium

Copper

Lead

Nickel

Selenium

Silver

Thallium

Vanadium

Zinc

Result Qualifier
12000
53000
7900
ND

$\mathbf{R L}$
2200
750
4100
7500

\begin{aligned} & Result Qualifier \\ & \cline { 2 - 2 } 0.44 \\ & 60 \\ & 300 \\ & 0.70 \\ &$N D \\ & 40 \\ & 77 \\ & 830 \\ & 55 \\ & 1.1 \\ & 0.19 \\ & N D \\ & 34 \\ & 93\end{aligned}$

\begin{tabular}{|c|c|c|}
\hline RL & MDL & Unit \\
\hline$\overline{0.18}$ & & $\overline{\mathrm{mg} / \mathrm{Kg}}$ \\
\hline 0.44 & & $\mathrm{mg} / \mathrm{Kg}$ \\
\hline 0.18 & & $\mathrm{mg} / \mathrm{Kg}$ \\
\hline 0.18 & & $\mathrm{mg} / \mathrm{Kg}$ \\
\hline 0.18 & & $\mathrm{mg} / \mathrm{Kg}$ \\
\hline 0.18 & & $\mathrm{mg} / \mathrm{Kg}$ \\
\hline 0.18 & & $\mathrm{mg} / \mathrm{Kg}$ \\
\hline 0.18 & & $\mathrm{mg} / \mathrm{Kg}$ \\
\hline 0.18 & & $\mathrm{mg} / \mathrm{Kg}$ \\
\hline 0.61 & & $\mathrm{mg} / \mathrm{Kg}$ \\
\hline 0.18 & & $\mathrm{mg} / \mathrm{Kg}$ \\
\hline 0.35 & & $\mathrm{mg} / \mathrm{Kg}$ \\
\hline 0.44 & & $\mathrm{mg} / \mathrm{Kg}$ \\
\hline 0.61 & & $\mathrm{mg} / \mathrm{Kg}$ \\
\hline
\end{tabular}

$\mathrm{mg} / \mathrm{Kg}$

Method: 7471A - Mercury (CVAA)

Analyte

Mercury

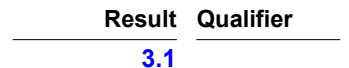

Result

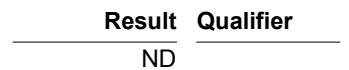

ND

$\begin{array}{ll}\text { MDL } & \text { Unit } \\ / \mathrm{Kg} \\ \mathrm{mg} / \mathrm{Kg} \\ \mathrm{mg} / \mathrm{Kg} \\ \mathrm{mg} / \mathrm{Kg}\end{array}$

\begin{tabular}{lll} 
D & Prepared \\
\cline { 2 - 2 } & $02 / 28 / 11$ & $10: 15$ \\
$02 / 28 / 11$ & $10: 15$ \\
$02 / 28 / 11$ & $10: 15$ \\
$02 / 28 / 11$ & $10: 15$
\end{tabular}

\begin{tabular}{|c|c|}
\hline Analyzed & Dil Fac \\
\hline 03/01/11 08:57 & 100 \\
\hline 03/01/11 08:57 & 100 \\
\hline 03/01/11 08:57 & 100 \\
\hline 03/01/11 08:57 & 100 \\
\hline
\end{tabular}

\begin{tabular}{c} 
Prepared \\
\hline 03/02/11 10:22 \\
03/02/11 10:22 \\
03/02/11 10:22 \\
\hline 03/02/11 10:22 \\
03/02/11 10:22 \\
03/02/11 10:22 \\
\hline 03/02/11 10:22 \\
03/02/11 10:22 \\
03/02/11 10:22 \\
\hline 03/02/11 10:22 \\
03/02/11 10:22 \\
03/02/11 10:22 \\
\hline $03 / 02 / 11 ~ 10: 22$ \\
$03 / 02 / 11 ~ 10: 22$ \\
\hline
\end{tabular}

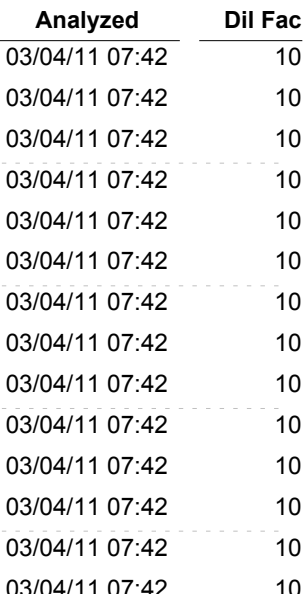

General Chemistry

Analyte

Cyanide, Total

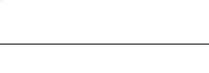

General Chemistry - Soluble

Analyte

Chloride

\begin{aligned} & Result Qualifier \\ & \hline 330 \\ & 1400\end{aligned}

\begin{tabular}{r}
$\mathbf{R L}$ \\
\hline 3.3 \\
30
\end{tabular}

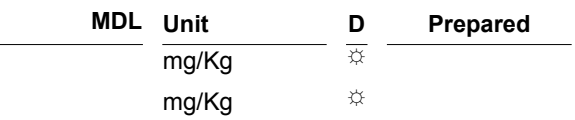

$\begin{array}{crr}\frac{\text { Analyzed }}{03 / 08 / 11} 13: 38 & & \text { Dil Fac } \\ 03 / 08 / 1117: 47 & & 10\end{array}$


Method: 6010B - Metals (ICP)

Analyte

\section{Aluminum}

Iron

Magnesium

Sodium

Method: 6020 - Metals (ICP/MS)

\section{Analyte}

Antimony

Arsenic

Barium

Beryllium

Cadmium

Chromium

Copper

Lead

Nickel

Selenium

Silver

Thallium

Vanadium

Zinc

$\begin{aligned} \text { Result } & \text { Qualifier } \\ & \\ 160000 & \\ 7500 & \\ \text { ND } & \end{aligned}$

\begin{tabular}{rr} 
Result & Qualifier \\
\cline { 2 - 2 } 0.70 & \\
41 & \\
190 \\
0.63 \\
0.48 \\
82 \\
93 \\
2800 \\
86 \\
3.1 \\
0.35 \\
$N D$ \\
38 \\
380
\end{tabular}

Method: 7471A - Mercury (CVAA)

Analyte

Mercury

$\mathbf{R L}$
2300
760
4200
7600

RL
2300
760
4200
7600

$\begin{array}{ll}\text { MDL } & \\ & \text { Unit } \\ \mathrm{mg} / \mathrm{Kg} \\ \mathrm{mg} / \mathrm{Kg} \\ \mathrm{mg} / \mathrm{Kg} \\ \mathrm{mg} / \mathrm{Kg}\end{array}$

D

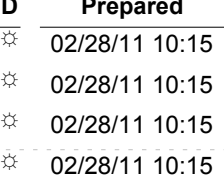

\begin{tabular}{|c|c|}
\hline Analyzed & Dil Fac \\
\hline 03/01/11 09:02 & 100 \\
\hline 03/01/11 09:02 & 100 \\
\hline 03/01/11 09:02 & 100 \\
\hline $03 / 01 / 1109: 02$ & 100 \\
\hline
\end{tabular}

\begin{tabular}{r}
$\mathbf{R L}$ \\
\hline 0.20 \\
0.49 \\
0.20 \\
0.20 \\
0.20 \\
0.20 \\
0.20 \\
20 \\
0.20 \\
0.69 \\
0.20 \\
0.39 \\
0.49 \\
0.69
\end{tabular}

MDL Unit

$\mathrm{mg} / \mathrm{Kg}$

$\mathrm{mg} / \mathrm{Kg}$

$\mathrm{mg} / \mathrm{Kg}$

$\mathrm{mg} / \mathrm{Kg}$

$\mathrm{mg} / \mathrm{Kg}$

$\mathrm{mg} / \mathrm{Kg}$

$\mathrm{mg} / \mathrm{Kg}$

$\mathrm{mg} / \mathrm{Kg}$

$\mathrm{mg} / \mathrm{Kg}$

$\mathrm{mg} / \mathrm{Kg}$

$\mathrm{mg} / \mathrm{Kg}$

$\mathrm{mg} / \mathrm{Kg}$

$\mathrm{mg} / \mathrm{Kg}$

$\mathrm{mg} / \mathrm{Kg}$

$\mathbf{R L}$
MDL Unit
$\frac{\text { Unit }}{\mathrm{mg} / \mathrm{Kg}}$

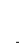
D Prepared 03/02/11 10:22 03/02/11 10:22 03/02/11 10:22 03/02/11 10:22 03/02/11 10:22 03/02/11 10:22 03/02/11 10:22 03/02/11 10:22 03/02/11 10:22 03/02/11 10:22 03/02/11 10:22 03/02/11 10:22 03/02/11 10:22 03/02/11 10:22

Dil Fac 03/04/11 07:46 10 03/04/11 07:46 03/04/11 07:46 03/04/11 07:46 03/04/11 07:46 03/04/11 07:46 $03 / 04 / 1107: 46$ 03/04/11 07:59 03/04/11 07:46 03/04/11 07:46 03/04/11 07:46 03/04/11 07:46 03/04/11 07:46 03/04/11 07:46

General Chemistry

Analyte

Cyanide, Total

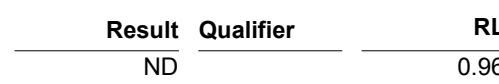

$\mathbf{R L}$

RL Unit $\frac{\text { Un }}{\mathrm{mg} / \mathrm{Kg}}$

$\frac{\text { D }}{3} \frac{\text { Prepared }}{02 / 24 / 1117: 04}$

$\frac{\text { Analyzed }}{02 / 28 / 1114: 39}$

Dil Fac

General Chemistry - Soluble

\begin{tabular}{|c|c|c|c|c|c|c|c|c|c|}
\hline Analyte & Result & Qualifier & RL & MDL & Unit & D & Prepared & Analyzed & Dil Fac \\
\hline Chloride & 190 & & 3.3 & & $\mathrm{mg} / \mathrm{Kg}$ & 票 & & 03/08/11 13:55 & 1 \\
\hline Sulfate & 6200 & & 150 & & $\mathrm{mg} / \mathrm{Kg}$ & 好 & & 03/09/11 11:10 & 50 \\
\hline
\end{tabular}

\section{Client Sample ID: S. Barrow 14 \#3- intermed. hole}

Date Collected: 02/17/11 15:30

Lab Sample ID: AUB0026-12

Date Received: 02/18/11 13:50

Matrix: Soil

Sampler Name:

Percent Solids: 98.9

Sampler Phone Number: (907) 272-1232

Method: 6010B - Metals (ICP)

\section{Analyte}

Aluminum

Iron

Magnesium

Sodium

Method: 6020 - Metals (ICP/MS)

Analyte

Antimony

Arsenic

$\begin{aligned} \text { Result } & \text { Qualifier } \\ & \\ 58000 & \\ 5300 & \\ \text { ND } & \end{aligned}$

\begin{tabular}{r}
$\mathbf{R L}$ \\
\hline 2300 \\
780 \\
4300 \\
7800
\end{tabular}

$\begin{array}{ll}\text { MDL } & \frac{\text { Unit }}{\mathrm{mg} / \mathrm{Kg}} \\ \mathrm{mg} / \mathrm{Kg} \\ \mathrm{mg} / \mathrm{Kg} \\ \mathrm{mg} / \mathrm{Kg}\end{array}$

$\mathrm{mg} / \mathrm{Kg}$ \begin{tabular}{ll}
$\frac{\text { D }}{n}$ & Prepared \\
\hline $02 / 28 / 11$ & $10: 15$ \\
$02 / 28 / 11$ & $10: 15$ \\
$02 / 28 / 11$ & $10: 15$ \\
\hline $02 / 28 / 11$ & $10: 15$
\end{tabular}

\begin{tabular}{ccr} 
Analyzed & & Dil Fac \\
\cline { 1 - 1 } 03/01/11 09:07 & & 100 \\
03/01/11 09:07 & & 100 \\
03/01/11 09:07 & & 100 \\
03/01/11 09:07 & & 100
\end{tabular}

$\frac{\text { RL }}{0.17} \stackrel{\text { MDL }}{\frac{\text { Unit }}{\mathrm{mg} / \mathrm{Kg}}} \underset{\mathrm{mg} / \mathrm{Kg}}{0.42}$

$\frac{\text { D }}{03 / 02 / 1110: 22}$

03/02/11 10:22

\begin{tabular}{|c|c|}
\hline Analyzed & Dil Fac \\
\hline $3 / 04 / 11$ 07:51 & \\
\hline $3 / 04$ & \\
\hline
\end{tabular}


Client Sample ID: S. Barrow 14 \#3- intermed. hole

Date Collected: 02/17/11 15:30

Date Received: 02/18/11 13:50

Sampler Name:
Lab Sample ID: AUB0026-12

Matrix: Soil

Percent Solids: 98.9

Sampler Phone Number: (907) 272-1232
Method: 6020 - Metals (ICP/MS) (Continued)

Analyte

Barium

Beryllium

Cadmium

Chromium

Copper

Lead

Nickel

Selenium

Silver

Thallium

Vanadium

Zinc

Method: 7471A - Mercury (CVAA)

Analyte

Mercury

Result Qualifier

0.98
Result Qualifier

75

0.45

ND

60

35

110

51

2.5

0.23

0.34

29

140
RL

0.17

0.17

0.17

0.17

0.17

0.17

0.58

0.17

0.33

0.42

0.58
MDL Unit $\mathrm{mg} / \mathrm{Kg}$

$\mathrm{mg} / \mathrm{Kg}$

$\mathrm{mg} / \mathrm{Kg}$

$\mathrm{mg} / \mathrm{Kg}$

$\mathrm{mg} / \mathrm{Kg}$

$\mathrm{mg} / \mathrm{Kg}$

$\mathrm{mg} / \mathrm{Kg}$

$\mathrm{mg} / \mathrm{Kg}$

$\mathrm{mg} / \mathrm{Kg}$

$\mathrm{mg} / \mathrm{Kg}$

$\mathrm{mg} / \mathrm{Kg}$

$\mathrm{mg} / \mathrm{Kg}$$$
\frac{D}{3}
$$$$
\frac{\text { Prepared }}{\text { 03/02/11 10:22 }}
$$$$
03 / 02 / 1110: 22
$$$$
\text { 03/02/11 10:22 }
$$$$
\text { 03/02/11 10:22 }
$$$$
\text { 03/02/11 10:22 }
$$$$
\text { 03/02/11 10:22 }
$$$$
\text { 03/02/11 10:22 }
$$$$
\text { 03/02/11 10:22 }
$$$$
\text { 03/02/11 10:22 }
$$$$
03 / 02 / 1110: 22
$$$$
\text { 03/02/11 10:22 }
$$$$
\text { 03/02/11 10:22 }
$$

\begin{tabular}{|c|c|}
\hline Analyzed & Dil Fac \\
\hline 03/04/11 07:51 & 10 \\
\hline 03/04/11 07:51 & 10 \\
\hline 03/04/11 07:51 & 10 \\
\hline 03/04/11 07:51 & 10 \\
\hline 03/04/11 07:51 & 10 \\
\hline 03/04/11 07:51 & 10 \\
\hline 03/04/11 07:51 & 10 \\
\hline 03/04/11 07:51 & 10 \\
\hline 03/04/11 07:51 & 10 \\
\hline 03/04/11 07:51 & 10 \\
\hline 03/04/11 07:51 & 10 \\
\hline 03/04/11 07:51 & 10 \\
\hline
\end{tabular}

\section{General Chemistry}

Analyte

Result Qualifier

ND

$\mathbf{R L}$

RL Unit

$\frac{\text { Unit }}{\mathrm{mg} / \mathrm{Kg}}$

D Prepared

Analyzed

Dil Fac

Cyanide, Total

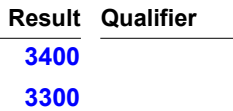

$\mathbf{R L}$
33
30

$\frac{\mathrm{RL}}{33}$

MDL Unit

$\mathrm{mg} / \mathrm{Kg}$

$\mathrm{mg} / \mathrm{Kg}$
$\mathrm{mg} / \mathrm{Kg}$

$\frac{D}{3}$
6

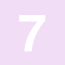

7
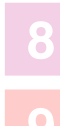

(0)
Chloride

Sulfate
General Chemistry - Soluble

3300

\section{$03 / 0$}

03/08/11 18:03 


\section{Quality Control Data}

Client: Petrotechnical Resources of Alaska

TestAmerica Job ID: AUB0026

Project/Site: [none]

Method: 6010B - Metals (ICP)

Lab Sample ID: MB 580-81322/19-A

Matrix: Solid

Client Sample ID: MB 580-81322/19-A

Analysis Batch: 81403

Prep Type: Total/NA

Prep Batch: 81322

Analyte

Aluminum

Iron

Magnesium

Sodium
MB MB

Result Qualifier

ND

ND

ND

ND

$\begin{array}{rll}\mathbf{R L} & \text { MDL } & \text { Unit } \\ 30 & \text { mg } / \mathrm{Kg} \\ 10 & \mathrm{mg} / \mathrm{Kg} \\ 55 & \mathrm{mg} / \mathrm{Kg} \\ 100 & \mathrm{mg} / \mathrm{Kg} & \end{array}$

$\underline{\text { D }}$

\begin{tabular}{|c|c|c|}
\hline rieparta & Analyzea & Dil rac \\
\hline 02/28/11 10:15 & 03/01/11 07:14 & 1 \\
\hline 02/28/11 10:15 & 03/01/11 07:14 & 1 \\
\hline 02/28/11 10:15 & 03/01/11 07:14 & 1 \\
\hline $02 / 28 / 11 \quad 10: 15$ & $03 / 01 / 1107: 14$ & 1 \\
\hline
\end{tabular}

Client Sample ID: LCS 580-81322/20-A

Lab Sample ID: LCS 580-81322/20-A

Matrix: Solid

Analysis Batch: 81403

\begin{tabular}{|c|c|c|c|}
\hline & $\begin{array}{l}\text { Spike } \\
\text { Added }\end{array}$ & $\begin{array}{r}\text { LCS } \\
\text { Result }\end{array}$ & $\begin{array}{l}\text { LCS } \\
\text { Qualifier }\end{array}$ \\
\hline Analyte & & Result & \\
\hline Aluminum & 200 & 204 & \\
\hline Iron & 1100 & 1070 & \\
\hline Magnesium & 1000 & 934 & \\
\hline Sodium & 1000 & 938 & \\
\hline
\end{tabular}

Lab Sample ID: LCSD 580-81322/21-A

Matrix: Solid

Analysis Batch: 81403

\section{Analyte}

Aluminum

Iron

Magnesium

Sodium

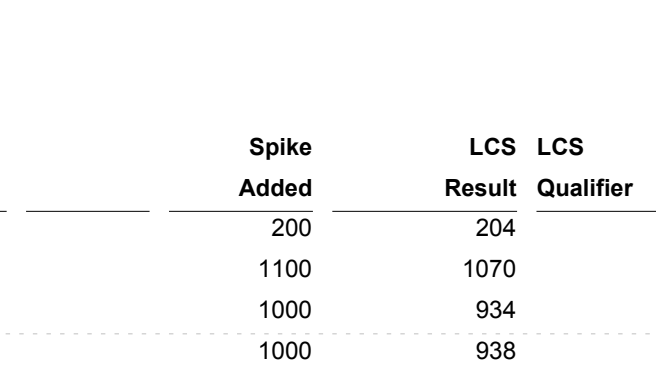

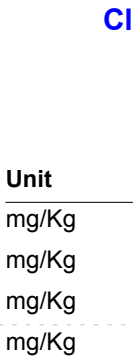
Prep Type: Total/NA Prep Batch: 81322 $\%$ Rec.

D \% Rec Limits

\begin{tabular}{lrr}
\hline $\mathrm{mg} / \mathrm{Kg}$ & -102 & $80-120$ \\
$\mathrm{mg} / \mathrm{Kg}$ & 98 & $80-120$ \\
$\mathrm{mg} / \mathrm{Kg}$ & 93 & $80-120$ \\
$\mathrm{mg} / \mathrm{Kg}$ & 94 & $80-120$
\end{tabular}

Client Sample ID: LCSD 580-81322/21-A Prep Type: Total/NA

Prep Batch: 81322 $\%$ Rec. RPD

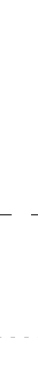

\begin{tabular}{|c|c|c|c|c|c|c|c|c|}
\hline Spike & LCSD & LCSD & & & & $\%$ Rec. & & RPD \\
\hline Added & Result & Qualifier & Unit & D & $\% \operatorname{Rec}$ & Limits & RPD & Limit \\
\hline 200 & 206 & & $\mathrm{mg} / \mathrm{Kg}$ & & 103 & $80-120$ & $\overline{1}$ & 20 \\
\hline 1100 & 1100 & & $\mathrm{mg} / \mathrm{Kg}$ & & 100 & $80-120$ & 2 & 20 \\
\hline 1000 & 920 & & $\mathrm{mg} / \mathrm{Kg}$ & & 92 & $80-120$ & 1 & 20 \\
\hline 1000 & 976 & & $\mathrm{mg} / \mathrm{Kg}$ & & 98 & $80-120$ & 4 & 20 \\
\hline
\end{tabular}

Method: 6020 - Metals (ICP/MS)

Lab Sample ID: MB 580-81476/22-A

Matrix: Solid

Client Sample ID: MB 580-81476/22-A

Analysis Batch: 81616

\begin{tabular}{|c|c|c|}
\hline Analyte & $\begin{array}{r}\text { MB } \\
\text { Result }\end{array}$ & $\begin{array}{l}\text { MB } \\
\text { Qualifier }\end{array}$ \\
\hline Antimony & $\overline{N D}$ & \\
\hline Arsenic & ND & \\
\hline Barium & ND & \\
\hline Beryllium & ND & \\
\hline Cadmium & ND & \\
\hline Chromium & ND & \\
\hline Copper & ND & \\
\hline Lead & ND & \\
\hline Nickel & ND & \\
\hline Selenium & ND & \\
\hline Silver & ND & \\
\hline Thallium & ND & \\
\hline Vanadium & ND & \\
\hline Zinc & ND & \\
\hline
\end{tabular}

Prep Type: Total/NA

Prep Batch: 81476

\begin{tabular}{|c|c|c|}
\hline RL & MDL & Unit \\
\hline 0.20 & & $\mathrm{mg} / \mathrm{Kg}$ \\
\hline 0.50 & & $\mathrm{mg} / \mathrm{Kg}$ \\
\hline 0.20 & & $\mathrm{mg} / \mathrm{Kg}$ \\
\hline 0.20 & & $\mathrm{mg} / \mathrm{Kg}$ \\
\hline 0.20 & & $\mathrm{mg} / \mathrm{Kg}$ \\
\hline 0.20 & & $\mathrm{mg} / \mathrm{Kg}$ \\
\hline 0.20 & & $\mathrm{mg} / \mathrm{Kg}$ \\
\hline 0.20 & & $\mathrm{mg} / \mathrm{Kg}$ \\
\hline 0.20 & & $\mathrm{mg} / \mathrm{Kg}$ \\
\hline 0.70 & & $\mathrm{mg} / \mathrm{Kg}$ \\
\hline 0.20 & & $\mathrm{mg} / \mathrm{Kg}$ \\
\hline 0.40 & & $\mathrm{mg} / \mathrm{Kg}$ \\
\hline 0.50 & & $\mathrm{mg} / \mathrm{Kg}$ \\
\hline 0.70 & & $\mathrm{mg} / \mathrm{Kg}$ \\
\hline
\end{tabular}

\begin{tabular}{cc} 
Prepared \\
\hline 03/02/11 $10: 22$ \\
$03 / 02 / 11$ & $10: 22$ \\
$03 / 02 / 11$ & $10: 22$ \\
$03 / 02 / 11$ & $10: 22$ \\
$03 / 02 / 11$ & $10: 22$ \\
$03 / 02 / 11$ & $10: 22$ \\
$03 / 02 / 11$ & $10: 22$ \\
$03 / 02 / 11$ & $10: 22$ \\
$03 / 02 / 11$ & $10: 22$ \\
$03 / 02 / 11$ & $10: 22$ \\
$03 / 02 / 11$ & $10: 22$ \\
$03 / 02 / 11$ & $10: 22$ \\
$03 / 02 / 11$ & $10: 22$ \\
$03 / 02 / 11$ & $10: 22$
\end{tabular}

\begin{tabular}{|c|c|}
\hline Analyzed & Dil Fac \\
\hline 03/03/11 14:49 & 10 \\
\hline 03/03/11 14:49 & 10 \\
\hline 03/03/11 14:49 & 10 \\
\hline $03 / 03 / 1114: 49$ & 10 \\
\hline 03/03/11 14:49 & 10 \\
\hline 03/03/11 14:49 & 10 \\
\hline 03/03/11 14:49 & 10 \\
\hline 03/03/11 14:49 & 10 \\
\hline 03/03/11 14:49 & 10 \\
\hline $03 / 03 / 1114: 49$ & 10 \\
\hline 03/03/11 14:49 & 10 \\
\hline 03/03/11 14:49 & 10 \\
\hline $03 / 03 / 1114: 49$ & 10 \\
\hline 03/03/11 14:49 & 10 \\
\hline
\end{tabular}




\section{Quality Control Data}

Client: Petrotechnical Resources of Alaska

\section{Method: 6020 - Metals (ICP/MS) (Continued)}

Lab Sample ID: LCS 580-81476/23-A

Matrix: Solid

Analysis Batch: 81616

\begin{tabular}{|c|c|c|c|}
\hline Analyte & $\begin{array}{r}\text { Spike } \\
\text { Added }\end{array}$ & $\begin{array}{r}\text { LCS } \\
\text { Result }\end{array}$ & $\begin{array}{l}\text { LCS } \\
\text { Qualifier }\end{array}$ \\
\hline Antimony & 150 & 152 & \\
\hline Arsenic & 200 & 211 & \\
\hline Barium & 200 & 212 & \\
\hline Beryllium & 5.00 & 4.93 & \\
\hline Cadmium & 5.00 & 5.42 & \\
\hline Chromium & 20.0 & 21.3 & \\
\hline Copper & 25.0 & 27.4 & \\
\hline Lead & 50.0 & 52.6 & \\
\hline Nickel & 50.0 & 53.6 & \\
\hline Selenium & 200 & 192 & \\
\hline Silver & 30.0 & 32.1 & \\
\hline Thallium & 200 & 202 & \\
\hline Vanadium & 50.0 & 53.4 & \\
\hline Zinc & 50.0 & 56.6 & \\
\hline
\end{tabular}

Lab Sample ID: LCSD 580-81476/24-A

Matrix: Solid

Analysis Batch: 81616

\begin{tabular}{|c|c|c|c|}
\hline Analyte & $\begin{array}{r}\text { Spike } \\
\text { Added }\end{array}$ & $\begin{array}{r}\text { LCSD } \\
\text { Result }\end{array}$ & $\begin{array}{l}\text { LCSD } \\
\text { Qualifier }\end{array}$ \\
\hline Antimony & 150 & 152 & \\
\hline Arsenic & 200 & 216 & \\
\hline Barium & 200 & 211 & \\
\hline Beryllium & 5.00 & 5.12 & \\
\hline Cadmium & 5.00 & 5.22 & \\
\hline Chromium & 20.0 & 21.5 & \\
\hline Copper & 25.0 & 27.9 & \\
\hline Lead & 50.0 & 52.9 & \\
\hline Nickel & 50.0 & 55.2 & \\
\hline Selenium & 200 & 210 & \\
\hline Silver & 30.0 & 32.0 & \\
\hline Thallium & 200 & 201 & \\
\hline Vanadium & 50.0 & 54.0 & \\
\hline Zinc & 50.0 & 57.8 & \\
\hline
\end{tabular}

Lab Sample ID: 580-24602-A-1-C MS

Matrix: Solid

Analysis Batch: 81616

\begin{tabular}{|c|c|c|}
\hline Analyte & $\begin{array}{r}\text { Sample } \\
\text { Result }\end{array}$ & $\begin{array}{l}\text { Sample } \\
\text { Qualifier }\end{array}$ \\
\hline Antimony & 2.4 & \\
\hline Arsenic & 4.3 & \\
\hline Barium & 160 & \\
\hline Beryllium & ND & \\
\hline Cadmium & 1.7 & \\
\hline Chromium & 14 & \\
\hline Copper & 170 & \\
\hline Lead & 51 & \\
\hline Nickel & 12 & \\
\hline Selenium & 2.2 & \\
\hline
\end{tabular}

Client Sample ID: LCS 580-81476/23-A

Prep Type: Total/NA

Prep Batch: 81476 $\%$ Rec.

Unit
$/ \mathrm{Kg}$
$\mathrm{mg} / \mathrm{Kg}$
$\mathrm{mg} / \mathrm{Kg}$
$\mathrm{mg} / \mathrm{Kg}$
$\mathrm{mg} / \mathrm{Kg}$
$\mathrm{mg} / \mathrm{Kg}$
$\mathrm{mg} / \mathrm{Kg}$
$\mathrm{mg} / \mathrm{Kg}$
$\mathrm{mg} / \mathrm{Kg}$
$\mathrm{mg} / \mathrm{Kg}$
$\mathrm{mg} / \mathrm{Kg}$
$\mathrm{mg} / \mathrm{Kg}$
$\mathrm{mg} / \mathrm{Kg}$
$\mathrm{mg} / \mathrm{Kg}$

D $\% \operatorname{Rec}$

Limits

$\begin{array}{ll}101 & 80-120 \\ 106 & 80-120\end{array}$

$10680-120$

$99 \quad 80-120$

$108 \quad 80-120$

$107 \quad 80-120$

$110 \quad 80-120$

$10580-120$

$107 \quad 80-120$

$96 \quad 80-120$

$107 \quad 80-120$

$10180-120$

$107 \quad 80-120$

$11380-120$

Client Sample ID: LCSD 580-81476/24-A Prep Type: Total/NA Prep Batch: 81476 $\%$ Rec.

D

\begin{tabular}{l} 
Unit \\
\cline { 1 - 1 } $\mathrm{mg} / \mathrm{Kg}$ \\
$\mathrm{mg} / \mathrm{Kg}$ \\
$\mathrm{mg} / \mathrm{Kg}$ \\
$\mathrm{mg} / \mathrm{Kg}$ \\
$\mathrm{mg} / \mathrm{Kg}$ \\
$\mathrm{mg} / \mathrm{Kg}$ \\
$\mathrm{mg} / \mathrm{Kg}$ \\
$\mathrm{mg} / \mathrm{Kg}$ \\
$\mathrm{mg} / \mathrm{Kg}$ \\
$\mathrm{mg} / \mathrm{Kg}$ \\
$\mathrm{mg} / \mathrm{Kg}$ \\
$\mathrm{mg} / \mathrm{Kg}$ \\
$\mathrm{mg} / \mathrm{Kg}$ \\
$\mathrm{mg} / \mathrm{Kg}$
\end{tabular}

\begin{aligned}$\%$ Rec \\ \hline 101 \\ 108 \\ 106 \\ 102 \\ 104 \\ 108 \\ 111 \\ 106 \\ 110 \\ 105 \\ 107 \\ 100 \\ 108 \\ 116\end{aligned}

Limits

RPD

$\frac{\text { Limits }}{80-120} \frac{\text { RPD }}{0} \frac{\text { Limit }}{20}$

$108 \quad 80-120 \quad 3 \quad 20$

$106 \quad 80-120 \quad 1 \quad 20$

$102 \quad 80-120 \quad 4 \quad 20$

$104 \quad 80-120 \quad 4 \quad 20$

$108 \quad 80-120 \quad 1 \quad 20$

$11180-120 \quad 2 \quad 20$

$106 \quad 80-120 \quad 1 \quad 20$

$110 \quad 80-120 \quad 3 \quad 20$

$105 \quad 80-120 \quad 9 \quad 20$

$107 \quad 80-120 \quad 0 \quad 20$

$100 \quad 80-120 \quad 1 \quad 20$

$108 \quad 80-120 \quad 1 \quad 20$

$116 \quad 80-120 \quad 2 \quad 20$

Client Sample ID: 580-24602-A-1-C MS Prep Type: Total/NA

Prep Batch: 81476 $\%$ Rec.

\begin{tabular}{rrr}
$\begin{array}{rr}\text { Spike } \\
\text { Added }\end{array}$ & $\begin{array}{r}\text { MS } \\
\text { Result }\end{array}$ & $\begin{array}{l}\text { MS } \\
\text { Qualifier }\end{array}$ \\
\cline { 2 - 3 } 335 & 330 & \\
446 & 473 & \\
446 & 627 & \\
111.2 & 13.1 \\
11.2 & 13.4 \\
44.6 & 62.2 \\
\hline 55.8 & 215 \\
112 & 168 \\
112 & 131 \\
\hline 446 & 476
\end{tabular}

\begin{tabular}{lrrr} 
Unit & D & \% Rec & \multicolumn{1}{l}{ Limits } \\
\cline { 4 - 4 } \cline { 4 - 4 } $\mathrm{mg} / \mathrm{Kg}$ & & 98 & $80-120$ \\
$\mathrm{mg} / \mathrm{Kg}$ & & 105 & $80-120$ \\
$\mathrm{mg} / \mathrm{Kg}$ & 104 & $80-120$ \\
$\mathrm{mg} / \mathrm{Kg}$ & 116 & $80-120$ \\
$\mathrm{mg} / \mathrm{Kg}$ & & 105 & $80-120$ \\
$\mathrm{mg} / \mathrm{Kg}$ & & 108 & $80-120$ \\
$\mathrm{mg} / \mathrm{Kg}$ & 87 & $80-120$ \\
$\mathrm{mg} / \mathrm{Kg}$ & & 105 & $80-120$ \\
$\mathrm{mg} / \mathrm{Kg}$ & & 106 & $80-120$ \\
$\mathrm{mg} / \mathrm{Kg}$ & 106 & $80-120$
\end{tabular}




\section{Quality Control Data}

Client: Petrotechnical Resources of Alaska

Project/Site: [none]

Method: 6020 - Metals (ICP/MS) (Continued)

Lab Sample ID: 580-24602-A-1-C MS

Matrix: Solid

Analysis Batch: 81616

\begin{tabular}{|c|c|c|}
\hline Analyte & $\begin{array}{r}\text { Sample } \\
\text { Result }\end{array}$ & $\begin{array}{l}\text { Sample } \\
\text { Qualifier }\end{array}$ \\
\hline Silver & 1.8 & \\
\hline Thallium & ND & \\
\hline Vanadium & 19 & \\
\hline Zinc & 380 & \\
\hline
\end{tabular}

Lab Sample ID: 580-24602-A-1-D MSD

Matrix: Solid

Analysis Batch: 81616

\begin{tabular}{lr} 
Analyte & $\begin{array}{r}\text { Sample } \\
\text { Result }\end{array} \begin{array}{l}\text { Sample } \\
\text { Qualifier }\end{array}$ \\
\cline { 1 - 2 } Antimony & 2.4 \\
Arsenic & 4.3 \\
Barium & 160 \\
Beryllium & $\mathrm{ND}$ \\
Cadmium & 1.7 \\
Chromium & 14 \\
Copper & 170 \\
Lead & 51 \\
Nickel & 12 \\
Selenium & 2.2 \\
Silver & 1.8 \\
Thallium & $\mathrm{ND}$ \\
Vanadium & 19 \\
Zinc & 380
\end{tabular}

Lab Sample ID: 580-24602-A-1-B DU

Matrix: Solid

Analysis Batch: 81616

\begin{tabular}{lrl} 
Analyte & $\begin{array}{r}\text { Sample } \\
\text { Result }\end{array}$ & $\begin{array}{l}\text { Sample } \\
\text { Qualifier }\end{array}$ \\
\cline { 1 - 2 } Antimony & 2.4 \\
Arsenic & 4.3 \\
Barium & 160 \\
Beryllium & $\mathrm{ND}$ \\
Cadmium & 1.7 \\
Chromium & 14 \\
Copper & 170 \\
Lead & 51 \\
Nickel & 12 \\
Selenium & 2.2 \\
Silver & 1.8 \\
Thallium & $\mathrm{ND}$ \\
Vanadium & 19 \\
Zinc & 380
\end{tabular}

Client Sample ID: 580-24602-A-1-C MS

Prep Type: Total/NA

Prep Batch: 81476 $\%$ Rec.

\begin{tabular}{|c|c|c|c|c|c|c|}
\hline $\begin{array}{l}\text { Spike } \\
\text { Added }\end{array}$ & $\begin{array}{r}\text { MS } \\
\text { Result }\end{array}$ & $\begin{array}{l}\text { MS } \\
\text { Qualifier }\end{array}$ & Unit & D & $\%$ R Rc & $\begin{array}{l}\% \text { Rec. } \\
\text { Limits }\end{array}$ \\
\hline 66.9 & 73.3 & & $\mathrm{mg} / \mathrm{Kg}$ & $\bar{\phi}$ & 107 & $80-120$ \\
\hline 446 & 450 & & $\mathrm{mg} / \mathrm{Kg}$ & 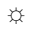 & 101 & $80-120$ \\
\hline 112 & 135 & & $\mathrm{mg} / \mathrm{Kg}$ & 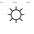 & 105 & $80-120$ \\
\hline 112 & 486 & & $\mathrm{mg} / \mathrm{Kg}$ & 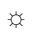 & 91 & $80-120$ \\
\hline
\end{tabular}

Client Sample ID: 580-24602-A-1-D MSD

Prep Type: Total/NA

Prep Batch: 81476

$\%$ Rec. RPD

Result Qualifier Unit D \% Rec Limits RPD Limit

$\begin{array}{lllllll}420 & 503 & \mathrm{mg} / \mathrm{Kg} & 119 & 80-120 & 6 & 20\end{array}$

$\begin{array}{lllllll}420 & 670 & \mathrm{mg} / \mathrm{Kg} & 120 & 80-120 & 7 & 20\end{array}$

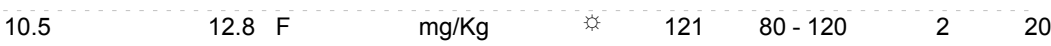

$\begin{array}{lllllll}10.5 & 13.9 & \mathrm{mg} / \mathrm{Kg} & 116 & 80-120 & 3 & 20\end{array}$

$\begin{array}{lllllll}42.0 & 65.6 \mathrm{~F} & \mathrm{mg} / \mathrm{Kg} & 122 & 80-120 & 5 & 20\end{array}$

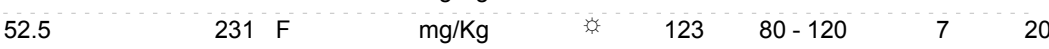

$\begin{array}{llllllll}105 & \mathrm{~F} & \mathrm{mg} / \mathrm{Kg} & 124 & 80-120 & 8 & 20\end{array}$

$105 \quad 142 \mathrm{~F} \quad \mathrm{mg} / \mathrm{Kg} \quad \begin{array}{rrrrr}124 & 80-120 & 8 & 20\end{array}$

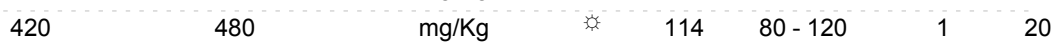

$\begin{array}{llllllll}63.0 & 78.2 & \mathrm{~F} & \mathrm{mg} / \mathrm{Kg} & 121 & 80-120 & 7 & 20\end{array}$

$\begin{array}{lllllll}420 & 484 & \mathrm{mg} / \mathrm{Kg} & 115 & 80-120 & 7 & 20\end{array}$

$105 \quad 144 \quad \begin{array}{lllll}120 & 80-120 & 7 & 20\end{array}$

$105 \quad 528 \mathrm{~F} \quad \mathrm{mg} / \mathrm{Kg} \quad \begin{array}{rrrrr}137 & 80-120 & 8 & 20\end{array}$

Client Sample ID: 580-24602-A-1-B DU

Prep Type: Total/NA

Prep Batch: 81476

DU DU

RPD

Result Qualifier

\begin{tabular}{|c|c|c|}
\hline esult & Qualifier & Unit \\
\hline 2.24 & & $\mathrm{mg} / \mathrm{Kg}$ \\
\hline 3.86 & & $\mathrm{mg} / \mathrm{Kg}$ \\
\hline 156 & & $\mathrm{mg} / \mathrm{Kg}$ \\
\hline ND & & $\mathrm{mg} / \mathrm{Kg}$ \\
\hline 1.43 & & $\mathrm{mg} / \mathrm{Kg}$ \\
\hline 13.0 & & $\mathrm{mg} / \mathrm{Kg}$ \\
\hline 153 & & $\mathrm{mg} / \mathrm{Kg}$ \\
\hline 47.7 & & $\mathrm{mg} / \mathrm{Kg}$ \\
\hline 11.1 & & $\mathrm{mg} / \mathrm{Kg}$ \\
\hline 1.96 & & $\mathrm{mg} / \mathrm{Kg}$ \\
\hline 1.67 & & $\mathrm{mg} / \mathrm{Kg}$ \\
\hline ND & & $\mathrm{mg} / \mathrm{Kg}$ \\
\hline 17.2 & & $\mathrm{mg} / \mathrm{Kg}$ \\
\hline 355 & & $\mathrm{mg} / \mathrm{Kg}$ \\
\hline
\end{tabular}

RPD Limit

$11 \quad 20$

520

NC 20

$15 \quad 20$

820

820

620

$7 \quad 20$

$12 \quad 20$

$6 \quad 20$

NC $\quad 20$

$\begin{array}{ll}7 & 20\end{array}$

820 


\section{Quality Control Data}

Client: Petrotechnical Resources of Alaska

Project/Site: [none]

Method: 7471A - Mercury (CVAA)

Lab Sample ID: MB 580-81552/22-A

Client Sample ID: MB 580-81552/22-A

Matrix: Solid

Prep Type: Total/NA

Analysis Batch: 81618

Prep Batch: 81552

Analyte

MB MB

Mercury

Result Qualifier

$\frac{\mathbf{R L}}{0.017} \longrightarrow \frac{\mathrm{MDL}}{\mathrm{mg} / \mathrm{Kg}}$

D

$\frac{\text { Prepared }}{03 / 03 / 1109: 03} \frac{\text { Analyzed }}{03 / 03 / 1111: 24} \frac{\text { Dil Fac }}{1}$

Lab Sample ID: LCS 580-81552/23-A

Client Sample ID: LCS 580-81552/23-A

Matrix: Solid

Prep Type: Total/NA

Analysis Batch: 81618

Analyte

Spike LCS LCS

Prep Batch: 81552

Mercury

Added Result Qualifier $\%$ Rec.

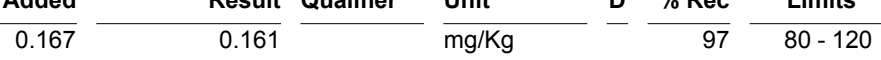

Lab Sample ID: LCSD 580-81552/24-A

Client Sample ID: LCSD 580-81552/24-A

Matrix: Solid

Prep Type: Total/NA

Analysis Batch: 81618

\begin{tabular}{|c|c|c|c|c|c|c|c|c|c|}
\hline Analyte & $\begin{array}{l}\text { Spike } \\
\text { Added }\end{array}$ & $\begin{array}{l}\text { LCSD } \\
\text { Result }\end{array}$ & $\begin{array}{l}\text { LCSD } \\
\text { Qualifier }\end{array}$ & Unit & D & $\% \operatorname{Rec}$ & $\begin{array}{l}\% \text { Rec. } \\
\text { Limits }\end{array}$ & RPD & $\begin{array}{l}\text { RPD } \\
\text { Limit }\end{array}$ \\
\hline Mercury & 0.167 & 0.163 & & $\overline{\mathrm{mg} / \mathrm{K}}$ & & 98 & $80-120$ & $\overline{1}$ & 20 \\
\hline
\end{tabular}

Lab Sample ID: 580-24645-A-1-D MS

Client Sample ID: 580-24645-A-1-D MS

Matrix: Solid

Analysis Batch: 81618

\begin{tabular}{|c|c|c|c|c|c|c|c|c|c|}
\hline 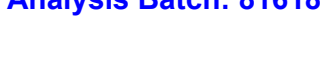 & Sample & Sample & Spike & MS & MS & & & & \% Rec. \\
\hline Analyte & Result & Qualifier & Added & Result & Qualifier & Unit & D & $\% \operatorname{Rec}$ & Limits \\
\hline Mercury & 0.14 & & 0.182 & 0.324 & & $\mathrm{mg} / \mathrm{Kg}$ & 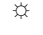 & 103 & $80-120$ \\
\hline
\end{tabular}

Lab Sample ID: 580-24645-A-1-E MSD

Client Sample ID: 580-24645-A-1-E MSD

Matrix: Solid

Prep Type: Total/NA

Analysis Batch: 81618

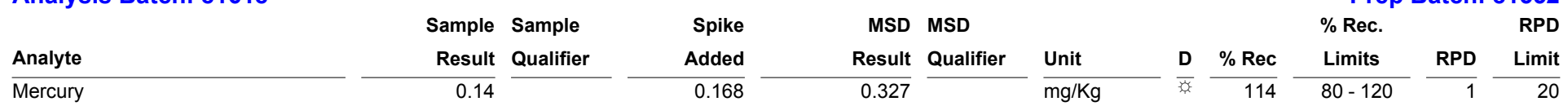

Lab Sample ID: 580-24645-A-1-C DU

Client Sample ID: 580-24645-A-1-C DU

Matrix: Solid

Prep Type: Total/NA

Analysis Batch: 81618

\begin{tabular}{|c|c|c|c|c|c|c|c|c|}
\hline 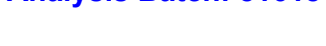 & Sample & Sample & DU & DU & & & & RPD \\
\hline Analyte & Result & Qualifier & Result & Qualifier & Unit & D & RPD & Limit \\
\hline Mercury & 0.14 & & 0.0957 & $\bar{F}$ & $\mathrm{mg} / \mathrm{Kg}$ & $\bar{x}$ & 35 & 20 \\
\hline
\end{tabular}

Method: 300.0 - Anions, lon Chromatography

Lab Sample ID: MB 580-81790/1-A

Client Sample ID: MB 580-81790/1-A

Matrix: Solid

Prep Type: Soluble

Analysis Batch: 81909

Analyte

Chloride

Sulfate
MB MB

ND

ND

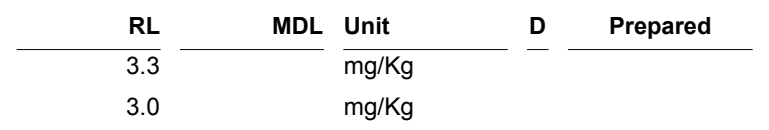

\begin{tabular}{|c|c|}
\hline Analyzed & Dil Fac \\
\hline 03/08/11 09:16 & 1 \\
\hline 03/08/11 09:16 & 1 \\
\hline
\end{tabular}
A 


\section{Quality Control Data}

Client: Petrotechnical Resources of Alaska

Project/Site: [none]

Method: 300.0 - Anions, Ion Chromatography (Continued)

Lab Sample ID: LCS 580-81790/2-A

Client Sample ID: LCS 580-81790/2-A

Matrix: Solid

Prep Type: Soluble

Analysis Batch: 81909

\begin{tabular}{|c|c|c|c|c|c|c|c|}
\hline Analyte & $\begin{array}{r}\text { Spike } \\
\text { Added }\end{array}$ & $\begin{array}{r}\text { LCS } \\
\text { Result }\end{array}$ & $\begin{array}{l}\text { LCS } \\
\text { Qualifier }\end{array}$ & Unit & D & $\% \operatorname{Rec}$ & $\begin{array}{l}\% \text { Rec. } \\
\text { Limits }\end{array}$ \\
\hline Chloride & 400 & 422 & & $\mathrm{mg} / \mathrm{Kg}$ & & 105 & $90-110$ \\
\hline Sulfate & 400 & 420 & & $\mathrm{mg} / \mathrm{Kg}$ & & 105 & $90-110$ \\
\hline
\end{tabular}

Lab Sample ID: 580-24607-1 MS

Client Sample ID: AUB0026-01

Matrix: Solid

Analysis Batch: 81909

\begin{tabular}{|c|c|c|c|c|c|c|c|c|c|}
\hline Analyte & $\begin{array}{r}\text { Sample } \\
\text { Result }\end{array}$ & $\begin{array}{l}\text { Sample } \\
\text { Qualifier }\end{array}$ & $\begin{array}{l}\text { Spike } \\
\text { Added }\end{array}$ & $\begin{array}{r}\text { MS } \\
\text { Result }\end{array}$ & $\begin{array}{l}\text { MS } \\
\text { Qualifier }\end{array}$ & Unit & D & $\% \operatorname{Rec}$ & $\begin{array}{l}\% \text { Rec. } \\
\text { Limits }\end{array}$ \\
\hline Chloride & 390 & & 401 & 188 & $\bar{F}$ & $\mathrm{mg} / \mathrm{Kg}$ & $\bar{x}$ & -51 & $90-110$ \\
\hline Sulfate & 210 & & 401 & 127 & $\mathrm{~F}$ & $\mathrm{mg} / \mathrm{Kg}$ & 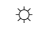 & -20 & $90-110$ \\
\hline
\end{tabular}

Lab Sample ID: 580-24607-2 MS

Client Sample ID: AUB0026-02

Matrix: Solid

Analysis Batch: 81909

\begin{tabular}{|c|c|c|c|c|c|c|c|c|c|}
\hline Analyte & $\begin{array}{r}\text { Sample } \\
\text { Result }\end{array}$ & $\begin{array}{l}\text { Sample } \\
\text { Qualifier }\end{array}$ & $\begin{array}{r}\text { Spike } \\
\text { Added }\end{array}$ & $\begin{array}{r}\text { MS } \\
\text { Result }\end{array}$ & $\begin{array}{l}\text { MS } \\
\text { Qualifier }\end{array}$ & Unit & D & $\% \operatorname{Rec}$ & $\begin{array}{l}\% \text { Rec. } \\
\text { Limits }\end{array}$ \\
\hline Chloride & 75 & & 395 & 513 & $\bar{F}$ & $\overline{\mathrm{mg} / \mathrm{Kg}}$ & $\bar{s}$ & $\overline{111}$ & $90-110$ \\
\hline Sulfate & 720 & & 395 & 925 & $\mathrm{~F}$ & $\mathrm{mg} / \mathrm{Kg}$ & 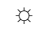 & 52 & $90-110$ \\
\hline
\end{tabular}

Lab Sample ID: 580-24607-1 DU

Client Sample ID: AUB0026-01

Matrix: Solid

Prep Type: Soluble

Analysis Batch: 81909

\begin{tabular}{|c|c|c|c|c|c|c|c|c|}
\hline & Sample & Sample & & & & & & $\begin{array}{l}\text { RPD } \\
\text { Limit }\end{array}$ \\
\hline Analyte & Result & Qualifier & Result & Qualifier & Unit & D & RPD & Limit \\
\hline Chloride & 390 & & 441 & $\bar{F}$ & $\mathrm{mg} / \mathrm{Kg}$ & $\bar{x}$ & 11 & 10 \\
\hline Sulfate & 210 & & 210 & & $\mathrm{mg} / \mathrm{Kg}$ & 禁 & 1 & 10 \\
\hline
\end{tabular}

\section{Method: 9012A - Cyanide, Total and/or Amenable}

Lab Sample ID: MB 580-81232/1-A

Matrix: Solid

Analysis Batch: 81358

Analyte

Cyanide, Total
MB MB

Result Qualifier

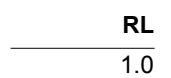

RL Unit

D

$\frac{\text { Prepared }}{02 / 24 / 1117: 04} \frac{\text { Analyzed }}{02 / 28 / 1114: 39} \frac{\text { Dil Fac }}{1}$

Client Sample ID: LCS 580-81232/2-A

Lab Sample ID: LCS 580-81232/2-A

Matrix: Solid

Analysis Batch: 81358

Analyte

Cyanide, Total

Spike

Added

10.0

LCS LCS

Result Qualifier

9.27

Client Sample ID: MB 580-81232/1-A

Prep Type: Total/NA

Prep Batch: 81232

Lab Sample ID: 580-24607-1 MS

Matrix: Solid

Analysis Batch: 81358

Analyte

Cyanide, Total

Sample Sample

$\begin{array}{r}\begin{array}{r}\text { Spike } \\ \text { Added }\end{array} \\ \hline 9.57\end{array}$

ND

9.57
Prep Type: Total/NA

Prep Batch: 81232 $\%$ Rec.

Limits

$80-120$

Client Sample ID: AUB0026-01

Prep Type: Total/NA

Prep Batch: 81232 $\%$ Rec.

Limits

$80-120$ 


\section{Quality Control Data}

Client: Petrotechnical Resources of Alaska

Project/Site: [none]

Method: 9012A - Cyanide, Total and/or Amenable (Continued)

Lab Sample ID: 580-24607-1 DU

Client Sample ID: AUB0026-01

Matrix: Solid

Prep Type: Total/NA

Analysis Batch: 81358

Analyte

Sample Sample

DU DU

Prep Batch: 81232

Cyanide, Total

Result Qualifier

Result Qualifier

Unit

D

$\frac{\text { RPD }}{\mathrm{NC}} \frac{\text { Limit }}{20}$ 


\section{QC Association Summary}

Client: Petrotechnical Resources of Alaska

Project/Site: [none]

\section{Metals}

Prep Batch: 81322

Lab Sample ID

AUB0026-04

AUB0026-05

AUB0026-06

AUB0026-07

AUB0026-08

AUB0026-09

AUB0026-10

AUB0026-11

AUB0026-12

MB 580-81322/19-A

LCS 580-81322/20-A

LCSD 580-81322/21-A

AUB0026-01

AUB0026-02

AUB0026-03

Analysis Batch: 81403

Lab Sample ID

MB 580-81322/19-A

AUB0026-03

AUB0026-04

AUB0026-05

AUB0026-06

AUB0026-07

AUB0026-08

AUB0026-09

AUB0026-10

AUB0026-11

AUB0026-12

LCS 580-81322/20-A

LCSD 580-81322/21-A

AUB0026-01

AUB0026-02

Prep Batch: 81476

Lab Sample ID

AUB0026-01

AUB0026-02

AUB0026-03

AUB0026-04

AUB0026-05

AUB0026-06

AUB0026-07

AUB0026-08

AUB0026-08

AUB0026-09

AUB0026-10

580-24602-A-1-B DU

AUB0026-11

AUB0026-11

AUB0026-12

MB 580-81476/22-A
Client Sample ID

Walakpa \#1 \#1- surface hole

Walakpa \#1 \#2- intermed. hole

Walakpa \#1 \#3- intermed. hole

S. Barrow $19 \# 1$ - surface hole

S. Barrow 19 \#2- intermed. hole

S. Barrow 19 \#3- intermed. hole

S. Barrow 14 \#1- surface hole

S. Barrow 14 \#2- intermed. hole

S. Barrow 14 \#3- intermed. hole

MB 580-81322/19-A

LCS 580-81322/20-A

LCSD 580-81322/21-A

Brontosaurus \#1 \#1- surface hole

Brontosaurus \#1 \#2- intermed hole

Brontosaurus \#1 \#3- intermed hole
Prep Type

Total/NA

Total/NA

Total/NA

Total/NA

Total/NA

Total/NA

Total/NA

Total/NA

Total/NA

Total/NA

Total/NA

Total/NA

Total/NA

Total/NA

Total/NA
Matrix

Soil

Soil

Soil

Soil

Soil

Soil

Soil

Soil

Soil

Solid

Solid

Solid

Soil

Soil

Soil
Method

3050B

3050B

3050B

3050B

3050B

3050B

3050B

3050B

3050B

3050B

3050B

3050B

3050B

3050B

3050B
Client Sample ID

MB 580-81322/19-A

Brontosaurus \#1 \#3- intermed hole

Walakpa \#1 \#1- surface hole

Walakpa \#1 \#2- intermed. hole

Walakpa \#1 \#3- intermed. hole

S. Barrow 19 \#1- surface hole

S. Barrow 19 \#2- intermed. hole

S. Barrow 19 \#3- intermed. hole

S. Barrow 14 \#1- surface hole

S. Barrow 14 \#2- intermed. hole

S. Barrow 14 \#3- intermed. hole

LCS 580-81322/20-A

LCSD 580-81322/21-A

Brontosaurus \#1 \#1- surface hole

Brontosaurus \#1 \#2- intermed hole
Prep Type

Total/NA

Total/NA

Total/NA

Total/NA

Total/NA

Total/NA

Total/NA

Total/NA

Total/NA

Total/NA

Total/NA

Total/NA

Total/NA

Total/NA

Total/NA
Matrix

Solid

Soil

Soil

Soil

Soil

Soil

Soil

Soil

Soil

Soil

Soil

Solid

Solid

Soil

Soil
Method

6010B

6010B

6010B

6010B

6010B

6010B

6010B

6010B

6010B

6010B

6010B

6010B

6010B

$6010 \mathrm{~B}$

6010B
Prep Batch

Prep Batch 81322

81322

81322

81322

81322

81322

81322

81322

81322

81322

81322

81322

81322

81322

81322
Client Sample ID

Brontosaurus \#1 \#1- surface hole

Brontosaurus \#1 \#2- intermed hole

Brontosaurus \#1 \#3- intermed hole

Walakpa \#1 \#1- surface hole

Walakpa \#1 \#2- intermed. hole

Walakpa \#1 \#3- intermed. hole

S. Barrow 19 \#1- surface hole

S. Barrow 19 \#2- intermed. hole

S. Barrow 19 \#2- intermed. hole

S. Barrow 19 \#3- intermed. hole

S. Barrow 14 \#1- surface hole

580-24602-A-1-B DU

S. Barrow 14 \#2- intermed. hole

S. Barrow 14 \#2- intermed. hole

S. Barrow 14 \#3- intermed. hole

MB 580-81476/22-A
Prep Type

Total/NA

Total/NA

Total/NA

Total/NA

Total/NA

Total/NA

Total/NA

Total/NA

Total/NA

Total/NA

Total/NA

Total/NA

Total/NA

Total/NA

Total/NA

Total/NA
Matrix

Soil

Soil

Soil

Soil

Soil

Soil

Soil

Soil

Soil

Soil

Soil

Solid

Soil

Soil

Soil

Solid

Method

3050B

3050B

3050B

3050B

3050B

3050B

3050B

3050B

3050B

3050B

3050B

3050B

3050B

3050B

3050B 


\section{QC Association Summary}

Client: Petrotechnical Resources of Alaska

Project/Site: [none]

\section{Metals (Continued)}

Prep Batch: 81476 (Continued)

Lab Sample ID

LCS 580-81476/23-A

LCSD 580-81476/24-A

580-24602-A-1-C MS

580-24602-A-1-D MSD

Prep Batch: 81552

Lab Sample ID

AUB0026-01

AUB0026-02

AUB0026-03

AUB0026-04

AUB0026-05

AUB0026-06

AUB0026-07

AUB0026-08

AUB0026-09

AUB0026-10

580-24645-A-1-C DU

AUB0026-11

AUB0026-12

MB 580-81552/22-A

LCS 580-81552/23-A

LCSD 580-81552/24-A

580-24645-A-1-D MS

580-24645-A-1-E MSD

Analysis Batch: 81616

Lab Sample ID

MB 580-81476/22-A

580-24602-A-1-B DU

580-24602-A-1-C MS

580-24602-A-1-D MSD

LCS 580-81476/23-A

LCSD 580-81476/24-A

AUB0026-01

AUB0026-02

AUB0026-03

AUB0026-04

AUB0026-05

AUB0026-06

AUB0026-07

Analysis Batch: 81618

Lab Sample ID

MB 580-81552/22-A

AUB0026-01

AUB0026-02

AUB0026-03

AUB0026-04

AUB0026-06

AUB0026-09

AUB0026-05
Client Sample ID

LCS 580-81476/23-A

LCSD 580-81476/24-A

580-24602-A-1-C MS

580-24602-A-1-D MSD
Prep Type

Total/NA

Total/NA

Total/NA

Total/NA
Matrix

Solid

Solid

Solid

Solid

Matrix

Brontosaurus \#1 \#1- surface hole

Brontosaurus \#1 \#2- intermed hole

Brontosaurus \#1 \#3- intermed hole

Walakpa \#1 \#1- surface hole

Walakpa \#1 \#2- intermed. hole

Walakpa \#1 \#3- intermed. hole

S. Barrow 19 \#1- surface hole

S. Barrow 19 \#2- intermed. hole

S. Barrow 19 \#3- intermed. hole

S. Barrow 14 \#1- surface hole

580-24645-A-1-C DU

S. Barrow 14 \#2- intermed. hole

S. Barrow 14 \#3- intermed. hole

MB 580-81552/22-A

LCS 580-81552/23-A

LCSD 580-81552/24-A

580-24645-A-1-D MS

580-24645-A-1-E MSD
Prep Type

Total/NA

Total/NA

Total/NA

Total/NA

Total/NA

Total/NA

Total/NA

Total/NA

Total/NA

Total/NA

Total/NA

Total/NA

Total/NA

Total/NA

Total/NA

Total/NA

Total/NA

Total/NA

\section{Soi}

Soil

Soil

Soil

Soil

Soil

Soil

Soil

Soil

Soil

Solid

Soil

Soil

Solid

Solid

Solid

Solid

Solid
Method

3050B

3050B

3050B

3050B

$7471 \mathrm{~A}$

$7471 \mathrm{~A}$

7471A

$7471 \mathrm{~A}$

$7471 \mathrm{~A}$

$7471 \mathrm{~A}$

$7471 \mathrm{~A}$

$7471 \mathrm{~A}$

$7471 \mathrm{~A}$

$7471 \mathrm{~A}$

$7471 \mathrm{~A}$

7471A

$7471 \mathrm{~A}$

$7471 \mathrm{~A}$

$7471 \mathrm{~A}$

$7471 \mathrm{~A}$

Client Sample ID

MB 580-81476/22-A

580-24602-A-1-B DU

580-24602-A-1-C MS

580-24602-A-1-D MSD

LCS 580-81476/23-A

LCSD 580-81476/24-A

Brontosaurus \#1 \#1- surface hole

Brontosaurus \#1 \#2- intermed hole

Brontosaurus \#1 \#3- intermed hole

Walakpa \#1 \#1- surface hole

Walakpa \#1 \#2- intermed. hole

Walakpa \#1 \#3- intermed. hole

S. Barrow $19 \# 1$ - surface hole
Prep Type

Total/NA

Total/NA

Total/NA

Total/NA

Total/NA

Total/NA

Total/NA

Total/NA

Total/NA

Total/NA

Total/NA

Total/NA

Total/NA
Matrix

Solid

Solid

Solid

Solid

Solid

Solid

Soil

Soil

Soil

Soil

Soil

Soil

Soil
Method

6020

6020

6020

6020

6020

6020

6020

6020

6020

6020

6020

6020

6020
Prep Batch

Prep Batch

Prep Batch

\section{9}

8

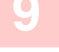




\section{QC Association Summary}

Client: Petrotechnical Resources of Alaska

Project/Site: [none]

\section{Metals (Continued)}

\section{Analysis Batch: 81618 (Continued)}

Lab Sample ID

LCS 580-81552/23-A

AUB0026-07

AUB0026-08

AUB0026-10

AUB0026-11

AUB0026-12

LCSD 580-81552/24-A

580-24645-A-1-C DU

580-24645-A-1-D MS

580-24645-A-1-E MSD

Analysis Batch: 81639

Lab Sample ID

AUB0026-08

AUB0026-09

AUB0026-10

AUB0026-11

AUB0026-12

AUB0026-08

AUB0026-11
Client Sample ID

LCS 580-81552/23-A

S. Barrow 19 \#1- surface hole

S. Barrow 19 \#2- intermed. hole

S. Barrow 14 \#1- surface hole

S. Barrow 14 \#2- intermed. hole

S. Barrow 14 \#3- intermed. hole

LCSD 580-81552/24-A

580-24645-A-1-C DU

580-24645-A-1-D MS

580-24645-A-1-E MSD
Prep Type

Total/NA

Total/NA

Total/NA

Total/NA

Total/NA

Total/NA

Total/NA

Total/NA

Total/NA

Total/NA
Matrix

Solid

Soil

Soil

Soil

Soil

Soil

Solid

Solid

Solid

Solid
Method

7471A

$7471 \mathrm{~A}$

7471A

7471A

$7471 \mathrm{~A}$

$7471 \mathrm{~A}$

7471A

7471A

7471A

7471A
Prep Batch

81552

81552

81552

81552

81552

81552

81552

81552

81552

81552
Client Sample ID

S. Barrow 19 \#2- intermed. hole

S. Barrow 19 \#3- intermed. hole

S. Barrow 14 \#1- surface hole

S. Barrow 14 \#2- intermed. hole

S. Barrow 14 \#3- intermed. hole

S. Barrow 19 \#2- intermed. hole

S. Barrow 14 \#2- intermed. hole
Prep Type

Total/NA

Total/NA

Total/NA

Total/NA

Total/NA

Total/NA

Total/NA

\section{Matrix}

Soil

Soil

Soil

Soil

Soil

Soil

Soil
Method

6020

6020

6020

6020

6020

6020

6020

\section{General Chemistry}

\section{Prep Batch: 81232}

Lab Sample ID

MB 580-81232/1-A

AUB0026-06

AUB0026-07

AUB0026-08

AUB0026-09

AUB0026-10

AUB0026-11

AUB0026-12

LCS 580-81232/2-A

AUB0026-01

580-24607-1 MS

580-24607-1 DU

AUB0026-02

AUB0026-03

AUB0026-04

AUB0026-05

Analysis Batch: 81335

Lab Sample ID

\section{AUB0026-01}

AUB0026-09

AUB0026-10

AUB0026-11

AUB0026-12

580-24607-1 DU

AUB0026-02

AUB0026-03

\section{Client Sample ID}

MB 580-81232/1-A

Walakpa \#1 \#3- intermed. hole

S. Barrow 19 \#1- surface hole

S. Barrow 19 \#2- intermed. hole

S. Barrow 19 \#3- intermed. hole

S. Barrow 14 \#1- surface hole

S. Barrow 14 \#2- intermed. hole

S. Barrow 14 \#3- intermed. hole

LCS 580-81232/2-A

Brontosaurus \#1 \#1- surface hole

AUB0026-01

AUB0026-01

Brontosaurus \#1 \#2- intermed hole

Brontosaurus \#1 \#3- intermed hole

Walakpa \#1 \#1- surface hole

Walakpa \#1 \#2- intermed. hole
Prep Type

Total/NA

Total/NA

Total/NA

Total/NA

Total/NA

Total/NA

Total/NA

Total/NA

Total/NA

Total/NA

Total/NA

Total/NA

Total/NA

Total/NA

Total/NA

Total/NA

$\begin{array}{lll}\text { Matrix } & \text { Method } & \text { Prep Batch } \\ \text { Solid } & 9012 \mathrm{~A} \\ \text { Soil } & 9012 \mathrm{~A} \\ \text { Soil } & 9012 \mathrm{~A} \\ \text { Soil } & 9012 \mathrm{~A} \\ \text { Soil } & 9012 \mathrm{~A} \\ \text { Soil } & 9012 \mathrm{~A} \\ \text { Soil } & 9012 \mathrm{~A} \\ \text { Soil } & 9012 \mathrm{~A} \\ \text { Solid } & 9012 \mathrm{~A} \\ \text { Soil } & 9012 \mathrm{~A} \\ \text { Solid } & 9012 \mathrm{~A} \\ \text { Solid } & 9012 \mathrm{~A} \\ \text { Soil } & 9012 \mathrm{~A} \\ \text { Soil } & 9012 \mathrm{~A} \\ \text { Soil } & 9012 \mathrm{~A} \\ \text { Soil } & 9012 \mathrm{~A}\end{array}$

Prep Type

Matrix

\section{Soil}

Soil

Soil

Soil

Soil

Solid

Soil

Soil
Method

Moisture

Moisture

Moisture

Moisture

Moisture

Moisture

Moisture

Moisture 


\section{QC Association Summary}

Client: Petrotechnical Resources of Alaska

Project/Site: [none]

\section{General Chemistry (Continued)}

\section{Analysis Batch: 81335 (Continued)}

\begin{tabular}{l} 
Lab Sample ID \\
\hline AUB0026-04 \\
AUB0026-05 \\
AUB0026-06 \\
AUB0026-07 \\
AUB0026-08
\end{tabular}

Client Sample ID

Walakpa \#1 \#1- surface hole

Walakpa \#1 \#2- intermed. hole

Walakpa \#1 \#3- intermed. hole

S. Barrow $19 \# 1$ - surface hole

S. Barrow 19 \#2- intermed. hole

\begin{tabular}{l} 
Prep Type \\
\hline Total/NA \\
Total/NA \\
Total/NA \\
Total/NA \\
Total/NA
\end{tabular}

Method

Moisture

Moisture

Moisture

Moisture

Moisture

\section{Analysis Batch: 81358}

Lab Sample ID

MB 580-81232/1-A

AUB0026-06

AUB0026-07

AUB0026-08

AUB0026-09

AUB0026-10

AUB0026-11

AUB0026-12

LCS 580-81232/2-A

AUB0026-01

580-24607-1 MS

580-24607-1 DU

AUB0026-02

AUB0026-03

AUB0026-04

AUB0026-05

Leach Batch: 81790

Lab Sample ID

MB 580-81790/1-A

AUB0026-05

AUB0026-06

AUB0026-06

AUB0026-07

AUB0026-08

AUB0026-09

AUB0026-10

AUB0026-10

AUB0026-11

AUB0026-11

AUB0026-12

LCS 580-81790/2-A

AUB0026-01

580-24607-1 MS

580-24607-1 DU

AUB0026-02

AUB0026-02

580-24607-2 MS

AUB0026-03

AUB0026-04

AUB0026-04
Client Sample ID

MB 580-81232/1-A

Walakpa \#1 \#3- intermed. hole

S. Barrow 19 \#1- surface hole

S. Barrow 19 \#2- intermed. hole

S. Barrow 19 \#3- intermed. hole

S. Barrow 14 \#1- surface hole

S. Barrow 14 \#2- intermed. hole

S. Barrow 14 \#3- intermed. hole

LCS 580-81232/2-A

Brontosaurus \#1 \#1- surface hole

AUB0026-01

AUB0026-01

Brontosaurus \#1 \#2- intermed hole

Brontosaurus \#1 \#3- intermed hole

Walakpa \#1 \#1- surface hole

Walakpa \#1 \#2- intermed. hole
Prep Type

Total/NA

Total/NA

Total/NA

Total/NA

Total/NA

Total/NA

Total/NA

Total/NA

Total/NA

Total/NA

Total/NA

Total/NA

Total/NA

Total/NA

Total/NA

Total/NA
Matrix

Solid

Soil

Soil

Soil

Soil

Soil

Soil

Soil

Solid

Soil

Solid

Solid

Soil

Soil

Soil

Soil
Method

9012A

9012A

9012A

9012A

9012A

9012A

$9012 \mathrm{~A}$

9012A

$9012 \mathrm{~A}$

$9012 \mathrm{~A}$

$9012 \mathrm{~A}$

$9012 \mathrm{~A}$

$9012 \mathrm{~A}$

$9012 \mathrm{~A}$

9012A

$9012 \mathrm{~A}$
Client Sample ID

MB 580-81790/1-A

Walakpa \#1 \#2- intermed. hole

Walakpa \#1 \#3- intermed. hole

Walakpa \#1 \#3- intermed. hole

S. Barrow 19 \#1- surface hole

S. Barrow 19 \#2- intermed. hole

S. Barrow 19 \#3- intermed. hole

S. Barrow 14 \#1- surface hole

S. Barrow 14 \#1- surface hole

S. Barrow 14 \#2- intermed. hole

S. Barrow 14 \#2- intermed. hole

S. Barrow 14 \#3- intermed. hole

LCS 580-81790/2-A

Brontosaurus \#1 \#1- surface hole

AUB0026-01

AUB0026-01

Brontosaurus \#1 \#2- intermed hole

Brontosaurus \#1 \#2- intermed hole

AUB0026-02

Brontosaurus \#1 \#3- intermed hole

Walakpa \#1 \#1- surface hole

Walakpa \#1 \#1- surface hole
Prep Type

Soluble

Soluble

Soluble

Soluble

Soluble

Soluble

Soluble

Soluble

Soluble

Soluble

Soluble

Soluble

Soluble

Soluble

Soluble

Soluble

Soluble

Soluble

Soluble

Soluble

Soluble

Soluble
Matrix

Solid

Soil

Soil

Soil

Soil

Soil

Soil

Soil

Soil

Soil

Soil

Soil

Solid

Soil

Solid

Solid

Soil

Soil

Solid

Soil

Soil

Soil
Method

DI Leach

DI Leach

DI Leach

DI Leach

DI Leach

DI Leach

DI Leach

DI Leach

DI Leach

DI Leach

DI Leach

DI Leach

DI Leach

DI Leach

DI Leach

DI Leach

DI Leach

DI Leach

DI Leach

DI Leach

DI Leach

DI Leach 


\section{QC Association Summary}

Client: Petrotechnical Resources of Alaska

Project/Site: [none]

\section{General Chemistry (Continued)}

\section{Analysis Batch: 81909}

Lab Sample ID
AUB0026-04
AUB0026-05
AUB0026-06
AUB0026-10
AUB0026-11
AUB0026-02
$580-24607-2$ MS
AUB0026-04
AUB0026-06
MB 580-81790/1-A
AUB0026-07
AUB0026-09
AUB0026-10
AUB0026-12
AUB0026-03
LCS 580-81790/2-A
AUB0026-08
AUB0026-11
AUB0026-01
$580-24607-1 \mathrm{MS}$
$580-24607-1 \mathrm{DU}$
AUB0026-02

Client Sample ID

Walakpa \#1 \#1- surface hole

Walakpa \#1 \#2- intermed. hole

Walakpa \#1 \#3- intermed. hole

S. Barrow 14 \#1- surface hole

S. Barrow 14 \#2- intermed. hole

Brontosaurus \#1 \#2- intermed hole

AUB0026-02

Walakpa \#1 \#1- surface hole

Walakpa \#1 \#3- intermed. hole

MB 580-81790/1-A

S. Barrow 19 \#1- surface hole

S. Barrow 19 \#3- intermed. hole

S. Barrow 14 \#1- surface hole

S. Barrow 14 \#3- intermed. hole

Brontosaurus \#1 \#3- intermed hole

LCS 580-81790/2-A

S. Barrow 19 \#2- intermed. hole

S. Barrow 14 \#2- intermed. hole

Brontosaurus \#1 \#1- surface hole

AUB0026-01

AUB0026-01

Brontosaurus \#1 \#2- intermed hole

\begin{tabular}{|c|c|c|c|}
\hline Prep Type & Matrix & Method & Prep Batch \\
\hline Soluble & Soil & 300.0 & \\
\hline Soluble & Soil & 300.0 & \\
\hline Soluble & Soil & 300.0 & \\
\hline Soluble & Soil & 300.0 & \\
\hline Soluble & Soil & 300.0 & \\
\hline Soluble & Soil & 300.0 & \\
\hline Soluble & Solid & 300.0 & \\
\hline Soluble & Soil & 300.0 & \\
\hline Soluble & Soil & 300.0 & \\
\hline Soluble & Solid & 300.0 & \\
\hline Soluble & Soil & 300.0 & \\
\hline Soluble & Soil & 300.0 & \\
\hline Soluble & Soil & 300.0 & \\
\hline Soluble & Soil & 300.0 & \\
\hline Soluble & Soil & 300.0 & \\
\hline Soluble & Solid & 300.0 & \\
\hline Soluble & Soil & 300.0 & \\
\hline Soluble & Soil & 300.0 & \\
\hline Soluble & Soil & 300.0 & \\
\hline Soluble & Solid & 300.0 & \\
\hline Soluble & Solid & 300.0 & \\
\hline Soluble & Soil & 300.0 & \\
\hline
\end{tabular}

8

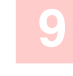




\section{Lab Chronicle}

Client: Petrotechnical Resources of Alaska

Project/Site: [none]

Client Sample ID: Brontosaurus \#1 \#1- surface hole

Lab Sample ID: AUB0026-01

Date Collected: 02/17/11 10:00

Matrix: Soil

Date Received: 02/18/11 13:50

Percent Solids: 99.2

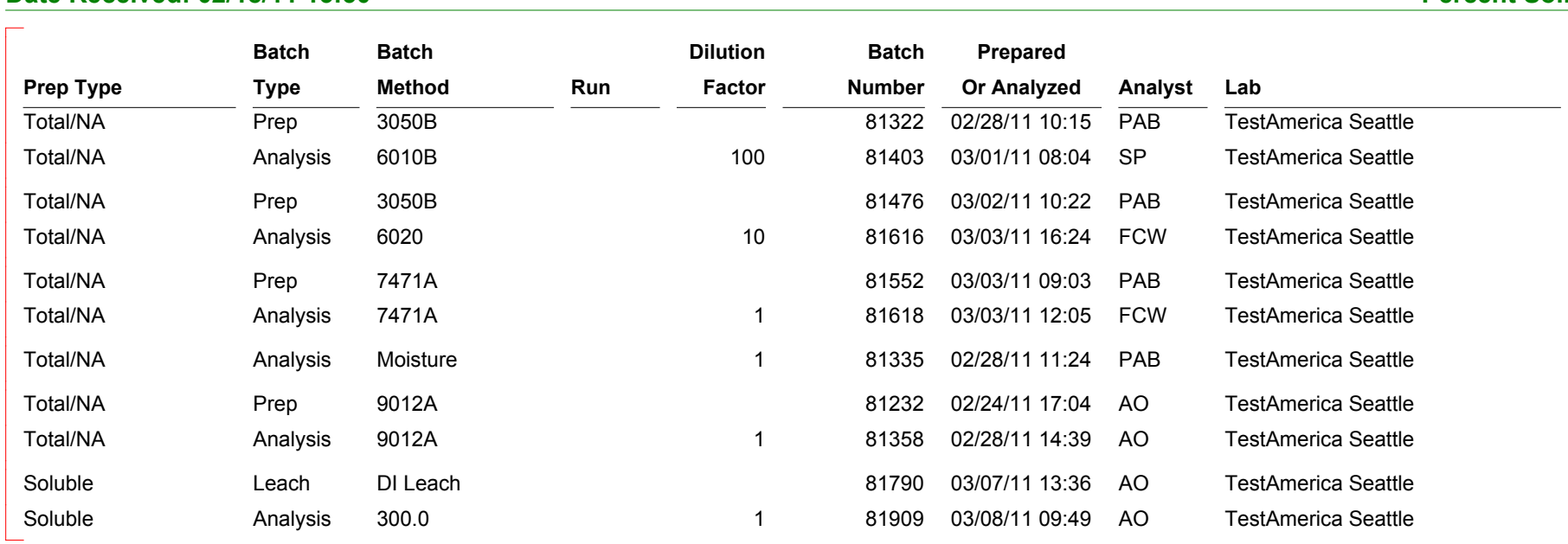

Client Sample ID: Brontosaurus \#1 \#2- intermed hole

Date Collected: 02/17/11 10:30

Lab Sample ID: AUB0026-02

Date Received: 02/18/11 13:50

Matrix: Soil

\begin{tabular}{|c|c|c|c|c|c|c|c|c|}
\hline Prep Type & $\begin{array}{l}\text { Batch } \\
\text { Type }\end{array}$ & $\begin{array}{l}\text { Batch } \\
\text { Method }\end{array}$ & Run & $\begin{array}{r}\text { Dilution } \\
\text { Factor }\end{array}$ & $\begin{array}{r}\text { Batch } \\
\text { Number }\end{array}$ & $\begin{array}{l}\text { Prepared } \\
\text { Or Analyzed }\end{array}$ & Analyst & Lab \\
\hline Total/NA & $\overline{\text { Prep }}$ & $3050 \mathrm{~B}$ & & & 81322 & 02/28/11 10:15 & $\overline{P A B}$ & TestAmerica Seattle \\
\hline Total/NA & Prep & $3050 \mathrm{~B}$ & & & 81476 & 03/02/11 10:22 & PAB & TestAmerica Seattle \\
\hline Total/NA & Analysis & 6020 & & 10 & 81616 & 03/03/11 16:36 & FCW & TestAmerica Seattle \\
\hline Total/NA & Analysis & $7471 \mathrm{~A}$ & & 1 & 81618 & 03/03/11 12:07 & FCW & TestAmerica Seattle \\
\hline Total/NA & Analysis & Moisture & & 1 & 81335 & 02/28/11 11:24 & PAB & TestAmerica Seattle \\
\hline Total/NA & Prep & $9012 \mathrm{~A}$ & & & 81232 & 02/24/11 17:04 & $\mathrm{AO}$ & TestAmerica Seattle \\
\hline Total/NA & Analysis & $9012 \mathrm{~A}$ & & 1 & 81358 & 02/28/11 14:39 & $\mathrm{AO}$ & TestAmerica Seattle \\
\hline Soluble & Leach & DI Leach & & & 81790 & 03/07/11 13:36 & $\mathrm{AO}$ & TestAmerica Seattle \\
\hline
\end{tabular}

Client Sample ID: Brontosaurus \#1 \#3- intermed hole

Lab Sample ID: AUB0026-03

Date Collected: 02/17/11 11:00

Matrix: Soil

Date Received: 02/18/11 13:50

Percent Solids: 99.1

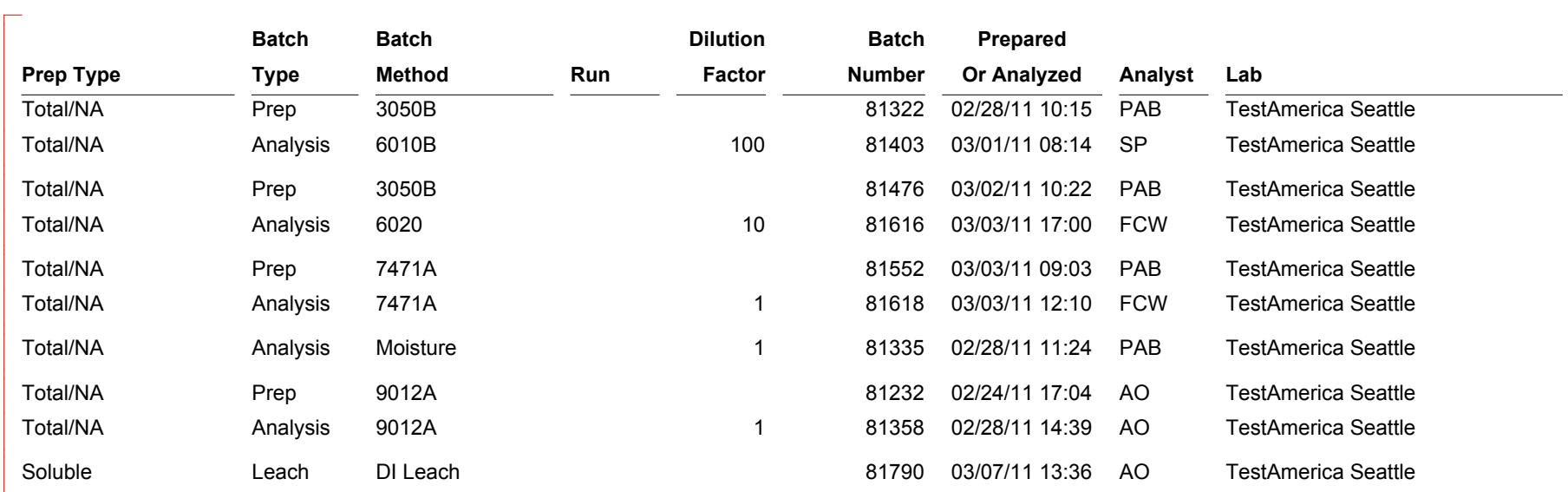

TestAmerica Anchorage 


\section{Lab Chronicle}

Client: Petrotechnical Resources of Alaska

Project/Site: [none]

Client Sample ID: Brontosaurus \#1 \#3- intermed hole

Lab Sample ID: AUB0026-03

Date Collected: 02/17/11 11:00

Matrix: Soil

Date Received: 02/18/11 13:50

Percent Solids: 99.1

\begin{tabular}{|c|c|c|c|c|c|c|c|c|}
\hline Soluble & Analysis & 300.0 & & $\overline{1}$ & 81909 & 03/08/11 18:19 & $\overline{\mathrm{AO}}$ & TestAmerica Seattle \\
\hline
\end{tabular}

\section{Client Sample ID: Walakpa \#1 \#1- surface hole}

Lab Sample ID: AUB0026-04

Date Collected: 02/17/11 11:30

Matrix: Soil

Date Received: 02/18/11 13:50

Percent Solids: 99.1

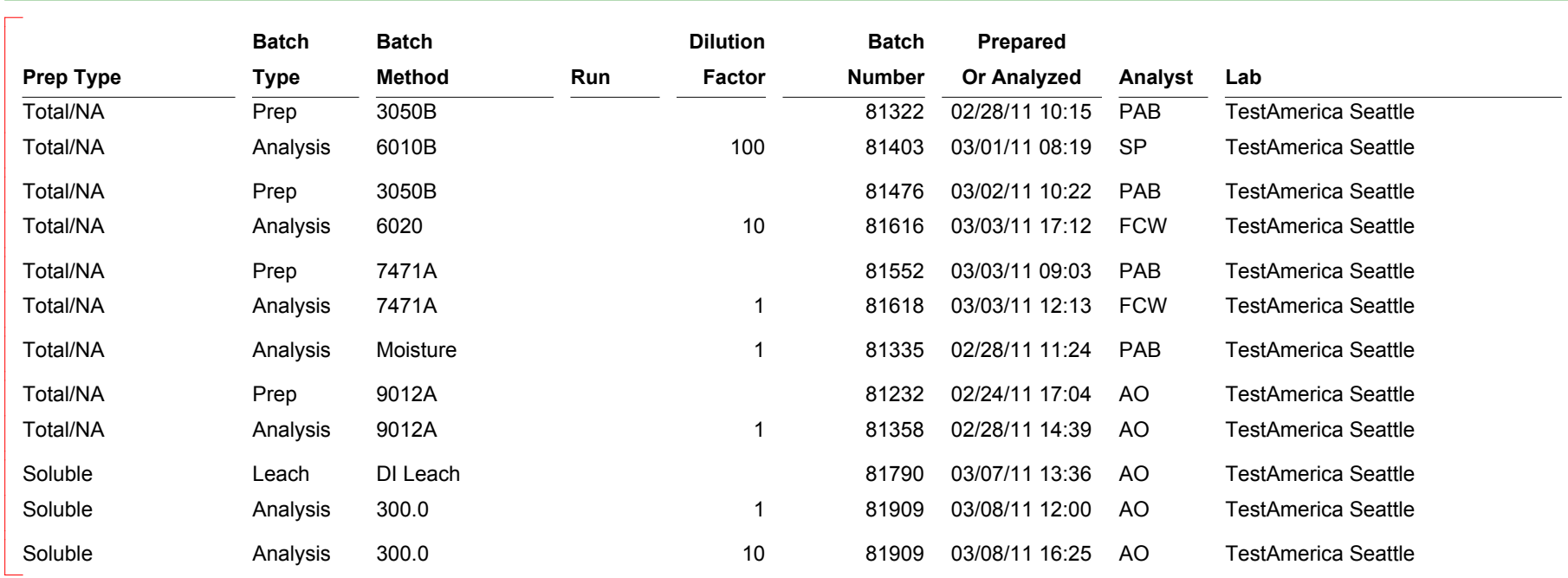

Client Sample ID: Walakpa \#1 \#2- intermed. hole

Lab Sample ID: AUB0026-05

Date Collected: 02/17/11 12:00

Matrix: Soil

Date Received: 02/18/11 13:50

Percent Solids: 99.2

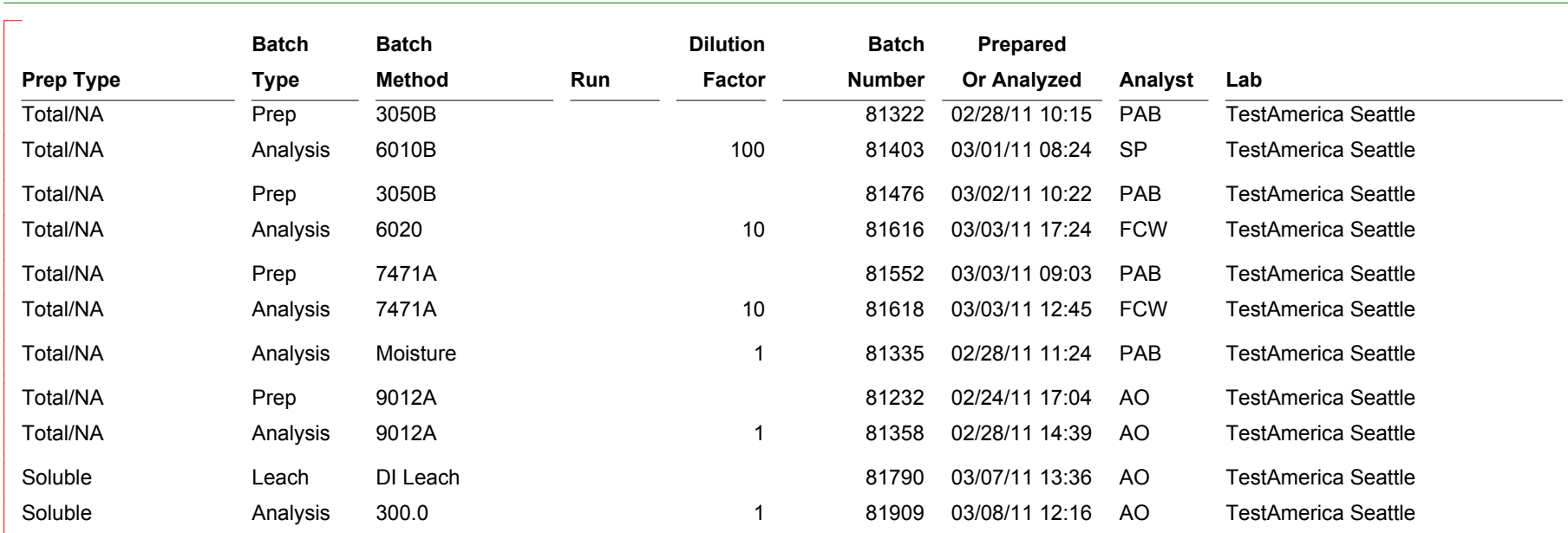

Client Sample ID: Walakpa \#1 \#3- intermed. hole

Lab Sample ID: AUB0026-06

Date Collected: 02/17/11 12:30

Matrix: Soil

Date Received: 02/18/11 13:50

Percent Solids: 98.6

\begin{tabular}{|c|c|c|c|c|c|c|c|c|}
\hline Prep Type & $\begin{array}{l}\text { Batch } \\
\text { Type }\end{array}$ & $\begin{array}{l}\text { Batch } \\
\text { Method }\end{array}$ & Run & $\begin{array}{r}\text { Dilution } \\
\text { Factor }\end{array}$ & $\begin{array}{r}\text { Batch } \\
\text { Number }\end{array}$ & $\begin{array}{l}\text { Prepared } \\
\text { Or Analyzed }\end{array}$ & Analyst & Lab \\
\hline Total/NA & $\overline{\text { Prep }}$ & 3050B & & & 81322 & 02/28/11 10:15 & $\overline{\mathrm{PAB}}$ & TestAmerica Seattle \\
\hline Total/NA & Prep & $3050 B$ & & & 81476 & 03/02/11 10:22 & PAB & TestAmerica Seattle \\
\hline
\end{tabular}




\section{Lab Chronicle}

Client: Petrotechnical Resources of Alaska

Project/Site: [none]

Client Sample ID: Walakpa \#1 \#3- intermed. hole

Lab Sample ID: AUB0026-06

Date Collected: 02/17/11 12:30

Matrix: Soil

Date Received: 02/18/11 13:50

Percent Solids: 98.6

\begin{tabular}{|c|c|c|c|c|c|c|c|c|}
\hline Total/NA & Analysis & 6020 & & 10 & 81616 & 03/03/11 17:36 & $\overline{\mathrm{FCW}}$ & TestAmerica Seattle \\
\hline Total/NA & Analysis & $7471 \mathrm{~A}$ & & 1 & 81618 & $03 / 03 / 1112: 25$ & FCW & TestAmerica Seattle \\
\hline Total/NA & Analysis & Moisture & & 1 & 81335 & $02 / 28 / 1111: 24$ & PAB & TestAmerica Seattle \\
\hline Soluble & Leach & DI Leach & & & 81790 & 03/07/11 13:36 & $\mathrm{AO}$ & TestAmerica Seattle \\
\hline Soluble & Analysis & 300.0 & & 1 & 81909 & $03 / 08 / 1112: 33$ & $\mathrm{AO}$ & TestAmerica Seattle \\
\hline Soluble & Analysis & 300.0 & & 10 & 81909 & 03/08/11 16:41 & $\mathrm{AO}$ & TestAmerica Seattle \\
\hline
\end{tabular}

Client Sample ID: S. Barrow 19 \#1- surface hole

Lab Sample ID: AUB0026-07

Date Collected: 02/17/11 13:00

Matrix: Soil

Date Received: 02/18/11 13:50

Percent Solids: 99.1

\begin{tabular}{|c|c|c|c|c|c|c|c|c|}
\hline Prep Type & $\begin{array}{l}\text { Batch } \\
\text { Type }\end{array}$ & $\begin{array}{l}\text { Batch } \\
\text { Method }\end{array}$ & Run & $\begin{array}{r}\text { Dilution } \\
\text { Factor }\end{array}$ & $\begin{array}{l}\text { Batch } \\
\text { Number }\end{array}$ & $\begin{array}{l}\text { Prepared } \\
\text { Or Analyzed }\end{array}$ & Analyst & Lab \\
\hline Total/NA & Prep & $3050 \mathrm{~B}$ & & & 81322 & 02/28/11 10:15 & $\overline{P A B}$ & TestAmerica Seattle \\
\hline Total/NA & Prep & $3050 \mathrm{~B}$ & & & 81476 & 03/02/11 10:22 & $\mathrm{PAB}$ & TestAmerica Seattle \\
\hline Total/NA & Analysis & 6020 & & 10 & 81616 & 03/03/11 17:48 & FCW & TestAmerica Seattle \\
\hline Total/NA & Analysis & Moisture & & 1 & 81335 & 02/28/11 11:24 & PAB & TestAmerica Seattle \\
\hline Total/NA & Prep & $9012 A$ & & & 81232 & 02/24/11 17:04 & $\mathrm{AO}$ & TestAmerica Seattle \\
\hline Total/NA & Analysis & $9012 \mathrm{~A}$ & & 1 & 81358 & 02/28/11 14:39 & $\mathrm{AO}$ & TestAmerica Seattle \\
\hline Soluble & Leach & DI Leach & & & 81790 & 03/07/11 13:36 & $\mathrm{AO}$ & TestAmerica Seattle \\
\hline Soluble & Analysis & 300.0 & & 10 & 81909 & 03/08/11 16:57 & $\mathrm{AO}$ & TestAmerica Seattle \\
\hline
\end{tabular}

Client Sample ID: S. Barrow 19 \#2- intermed. hole

Lab Sample ID: AUB0026-08

Date Collected: 02/17/11 13:30

Matrix: Soil

Date Received: 02/18/11 13:50

Percent Solids: 97.3

\begin{tabular}{|c|c|c|c|c|c|c|c|c|}
\hline Prep Type & $\begin{array}{l}\text { Batch } \\
\text { Type }\end{array}$ & $\begin{array}{l}\text { Batch } \\
\text { Method }\end{array}$ & Run & $\begin{array}{r}\text { Dilution } \\
\text { Factor }\end{array}$ & $\begin{array}{r}\text { Batch } \\
\text { Number }\end{array}$ & $\begin{array}{l}\text { Prepared } \\
\text { Or Analyzed }\end{array}$ & Analyst & Lab \\
\hline Total/NA & Prep & $3050 \mathrm{~B}$ & & & 81322 & 02/28/11 10:15 & $\overline{\mathrm{PAB}}$ & TestAmerica Seattle \\
\hline Total/NA & Analysis & $6010 B$ & & 100 & 81403 & 03/01/11 08:38 & $\mathrm{SP}$ & TestAmerica Seattle \\
\hline Total/NA & Prep & $7471 \mathrm{~A}$ & & & 81552 & 03/03/11 09:03 & $\mathrm{PAB}$ & TestAmerica Seattle \\
\hline Total/NA & Analysis & $7471 \mathrm{~A}$ & & 10 & 81618 & $03 / 03 / 1112: 51$ & FCW & TestAmerica Seattle \\
\hline Total/NA & Prep & $3050 \mathrm{~B}$ & & & 81476 & $03 / 02 / 11$ 10:22 & $\mathrm{PAB}$ & TestAmerica Seattle \\
\hline Total/NA & Analysis & 6020 & & 1000 & 81639 & 03/04/11 07:55 & FCW & TestAmerica Seattle \\
\hline Total/NA & Analysis & Moisture & & 1 & 81335 & 02/28/11 11:24 & $\mathrm{PAB}$ & TestAmerica Seattle \\
\hline Total/NA & Prep & $9012 \mathrm{~A}$ & & & 81232 & 02/24/11 17:04 & $\mathrm{AO}$ & TestAmerica Seattle \\
\hline Total/NA & Analysis & $9012 \mathrm{~A}$ & & 1 & 81358 & 02/28/11 14:39 & $\mathrm{AO}$ & TestAmerica Seattle \\
\hline Soluble & Leach & DI Leach & & & 81790 & 03/07/11 13:36 & $\mathrm{AO}$ & TestAmerica Seattle \\
\hline
\end{tabular}




\section{Lab Chronicle}

Client: Petrotechnical Resources of Alaska

Project/Site: [none]

Client Sample ID: S. Barrow 19 \#3- intermed. hole

Lab Sample ID: AUB0026-09

Date Collected: 02/17/11 14:00

Matrix: Soil

Date Received: 02/18/11 13:50

Percent Solids: 98.0

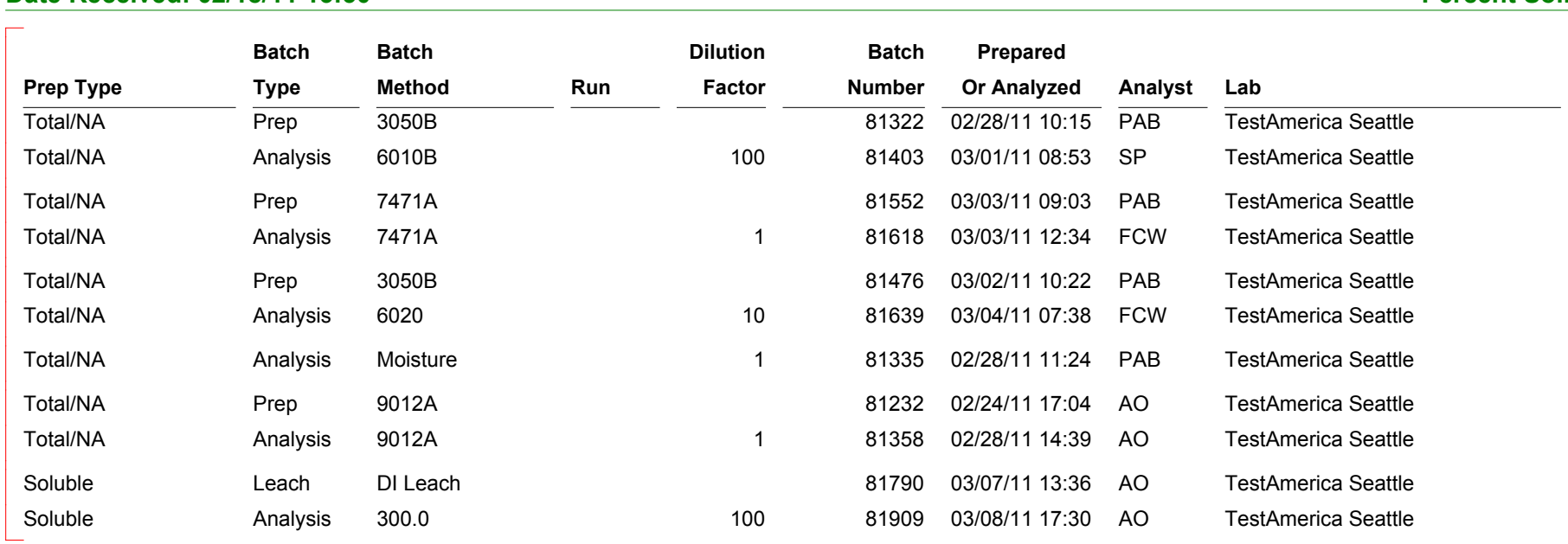

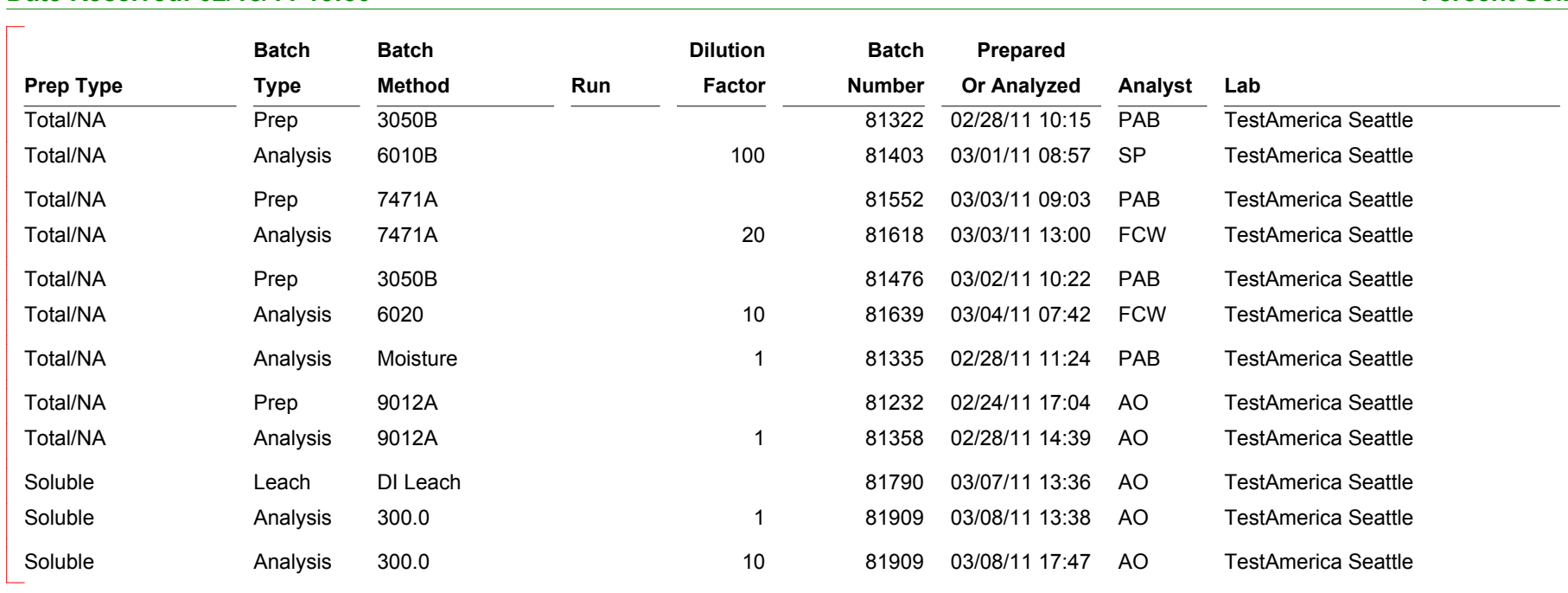

Client Sample ID: S. Barrow 14 \#2- intermed. hole

Lab Sample ID: AUB0026-11

Date Collected: 02/17/11 15:00

Matrix: Soil

Date Received: 02/18/11 13:50

Percent Solids: 98.9

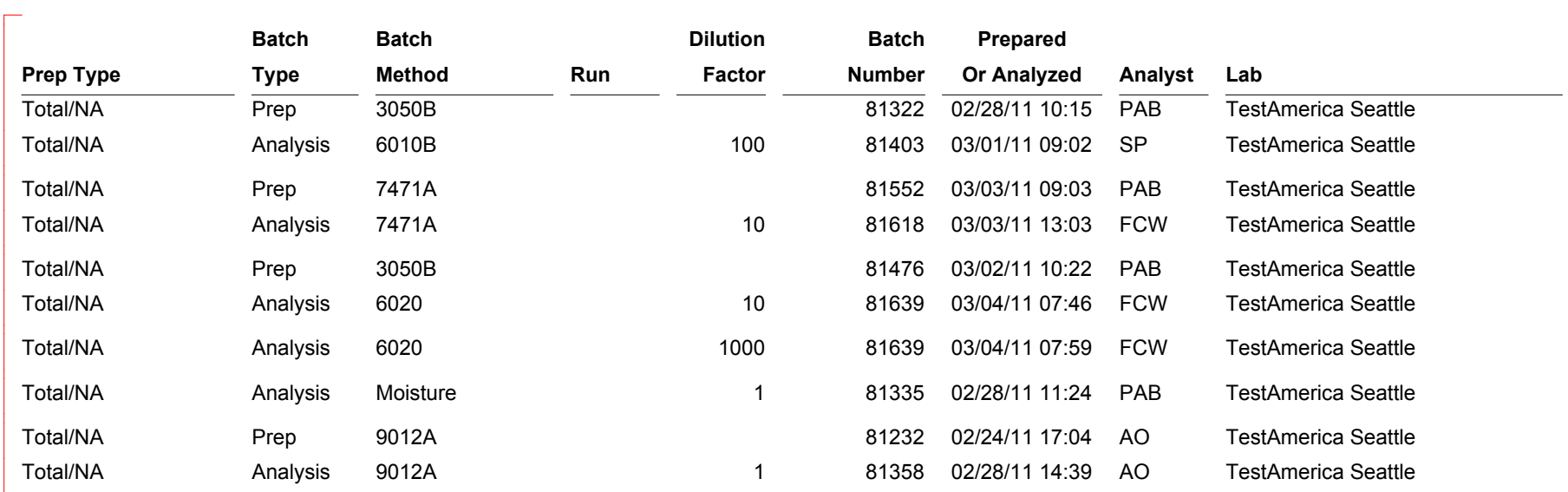




\section{Lab Chronicle}

Client: Petrotechnical Resources of Alaska

Project/Site: [none]

Client Sample ID: S. Barrow 14 \#2- intermed. hole

Lab Sample ID: AUB0026-11

Date Collected: 02/17/11 15:00

Matrix: Soil

Date Received: 02/18/11 13:50

Percent Solids: 98.9

\begin{tabular}{|c|c|c|c|c|c|c|c|c|}
\hline Soluble & Leach & DI Leach & & & 81790 & 03/07/11 13:36 & $\overline{\mathrm{AO}}$ & TestAmerica Seattle \\
\hline Soluble & Analysis & 300.0 & & 50 & 81909 & 03/09/11 11:10 & $\mathrm{AO}$ & TestAmerica Seattle \\
\hline
\end{tabular}

Client Sample ID: S. Barrow 14 \#3- intermed. hole

Date Collected: 02/17/11 15:30

Lab Sample ID: AUB0026-12

Date Received: 02/18/11 13:50

Matrix: Soil

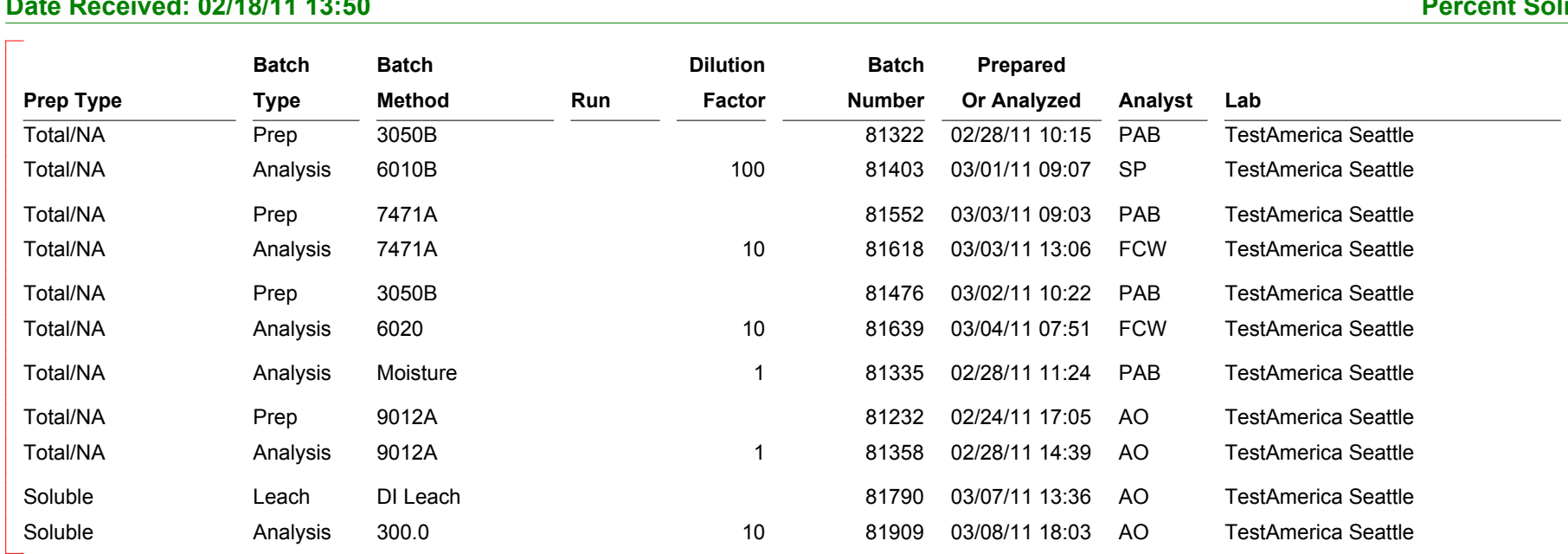




\section{Certification Summary}

Client: Petrotechnical Resources of Alaska

TestAmerica Job ID: AUB0026

Project/Site: [none]

\begin{tabular}{|c|c|c|c|c|c|}
\hline Laboratory & Authority & Program & EPA Region & Certification ID & * Expiration Date \\
\hline TestAmerica Anchorage & Alaska & Alaska UST & 10 & UST-067 & $06 / 16 / 11$ \\
\hline TestAmerica Anchorage & Alaska & State Program & 10 & AK00975 & $06 / 30 / 11$ \\
\hline TestAmerica Seattle & & USDA & & P330-08-00099 & $05 / 21 / 11$ \\
\hline TestAmerica Seattle & Alaska & Alaska UST & 10 & UST-022 & $03 / 03 / 11$ \\
\hline TestAmerica Seattle & California & NELAC & 9 & $1115 \mathrm{CA}$ & $01 / 31 / 12$ \\
\hline TestAmerica Seattle & Florida & NELAC & 4 & E871074 & 06/30/11 \\
\hline TestAmerica Seattle & L-A-B & DoD ELAP & 0 & L2236 & $01 / 19 / 13$ \\
\hline TestAmerica Seattle & L-A-B & ISO/IEC 17025 & 0 & L2236 & 01/19/13 \\
\hline TestAmerica Seattle & Louisiana & NELAC Secondary AB & 6 & 05016 & $06 / 30 / 11$ \\
\hline TestAmerica Seattle & Montana & MT DEQ UST & 8 & & $04 / 30 / 20$ \\
\hline TestAmerica Seattle & Oregon & NELAC & 10 & WA100007 & $11 / 06 / 11$ \\
\hline TestAmerica Seattle & Washington & State Program & 10 & C553 & $02 / 16 / 12$ \\
\hline
\end{tabular}

Accreditation may not be offered or required for all methods and analytes reported in this package. Please contact your project manager for the laboratory's current list of certified methods and analytes.

* Any expired certifications in this list are currently pending renewal and are considered valid. 


\section{Method Summary}

Client: Petrotechnical Resources of Alaska

Project/Site: [none]

\begin{tabular}{|c|c|c|c|}
\hline Method & Method Description & Protocol & Laboratory \\
\hline $6010 B$ & Metals (ICP) & SW846 & TAL SEA \\
\hline 6020 & Metals (ICP/MS) & SW846 & TAL SEA \\
\hline $7471 \mathrm{~A}$ & Mercury (CVAA) & SW846 & TAL SEA \\
\hline 300.0 & Anions, Ion Chromatography & MCAWW & TAL SEA \\
\hline $9012 \mathrm{~A}$ & Cyanide, Total and/or Amenable & SW846 & TAL SEA \\
\hline Moisture & Percent Moisture & EPA & TAL SEA \\
\hline
\end{tabular}

Protocol References:

$\mathrm{EPA}=$ US Environmental Protection Agency

MCAWW = "Methods For Chemical Analysis Of Water And Wastes", EPA-600/4-79-020, March 1983 And Subsequent Revisions.

SW846 = "Test Methods For Evaluating Solid Waste, Physical/Chemical Methods", Third Edition, November 1986 And Its Updates.

Laboratory References:

TAL SEA = TestAmerica Seattle, 5755 8th Street East, Tacoma, WA 98424, TEL (253)922-2310 


\section{Sample Summary}

Client: Petrotechnical Resources of Alaska

Project/Site: [none]

\begin{tabular}{|c|c|c|c|c|}
\hline Lab Sample ID & Client Sample ID & Matrix & Collected & Received \\
\hline AUB0026-01 & Brontosaurus \#1 \#1- surface hole & Soil & 02/17/1110:00 & 02/18/11 13:50 \\
\hline AUB0026-02 & Brontosaurus \#1 \#2- intermed hole & Soil & 02/17/11 10:30 & 02/18/11 13:50 \\
\hline AUB0026-03 & Brontosaurus \#1 \#3- intermed hole & Soil & 02/17/11 11:00 & 02/18/11 13:50 \\
\hline AUB0026-04 & Walakpa \#1 \#1- surface hole & Soil & $02 / 17 / 1111: 30$ & $02 / 18 / 1113: 50$ \\
\hline AUB0026-05 & Walakpa \#1 \#2- intermed. hole & Soil & 02/17/11 12:00 & 02/18/11 13:50 \\
\hline AUB0026-06 & Walakpa \#1 \#3- intermed. hole & Soil & 02/17/11 12:30 & 02/18/11 13:50 \\
\hline AUB0026-07 & S. Barrow $19 \# 1$ - surface hole & Soil & 02/17/111 13:00 & $02 / 18 / 1113: 50$ \\
\hline AUB0026-08 & S. Barrow 19 \#2- intermed. hole & Soil & 02/17/11 13:30 & 02/18/11 13:50 \\
\hline AUB0026-09 & S. Barrow 19 \#3- intermed. hole & Soil & 02/17/11 14:00 & 02/18/11 13:50 \\
\hline AUB0026-10 & S. Barrow $14 \# 1$ - surface hole & Soil & 02/17/111 14:30 & $02 / 18 / 1113: 50$ \\
\hline AUB0026-11 & S. Barrow 14 \#2- intermed. hole & Soil & 02/17/11 15:00 & 02/18/11 13:50 \\
\hline AUB0026-12 & S. Barrow 14 \#3- intermed. hole & Soil & 02/17/11 15:30 & 02/18/11 13:50 \\
\hline
\end{tabular}




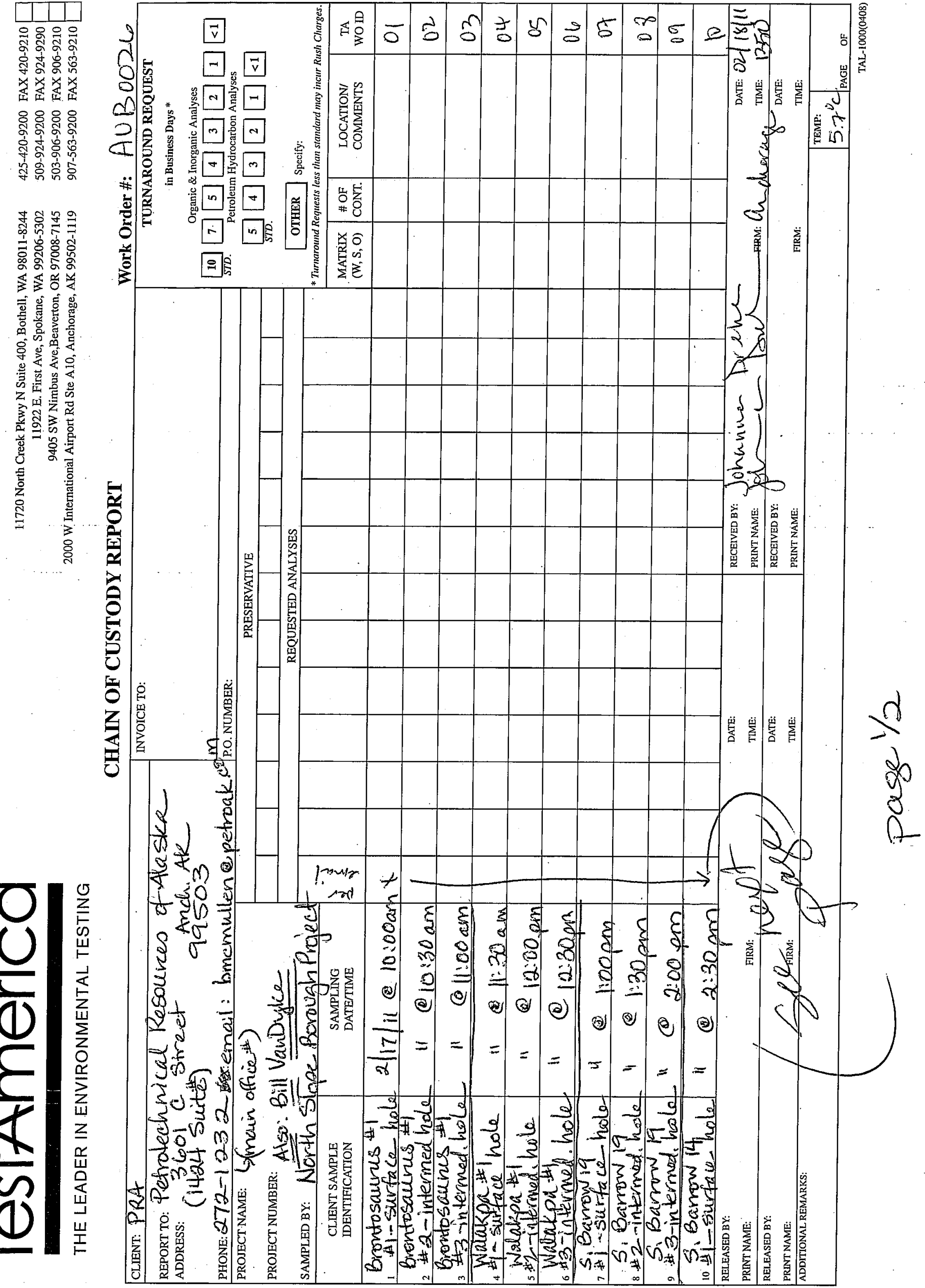




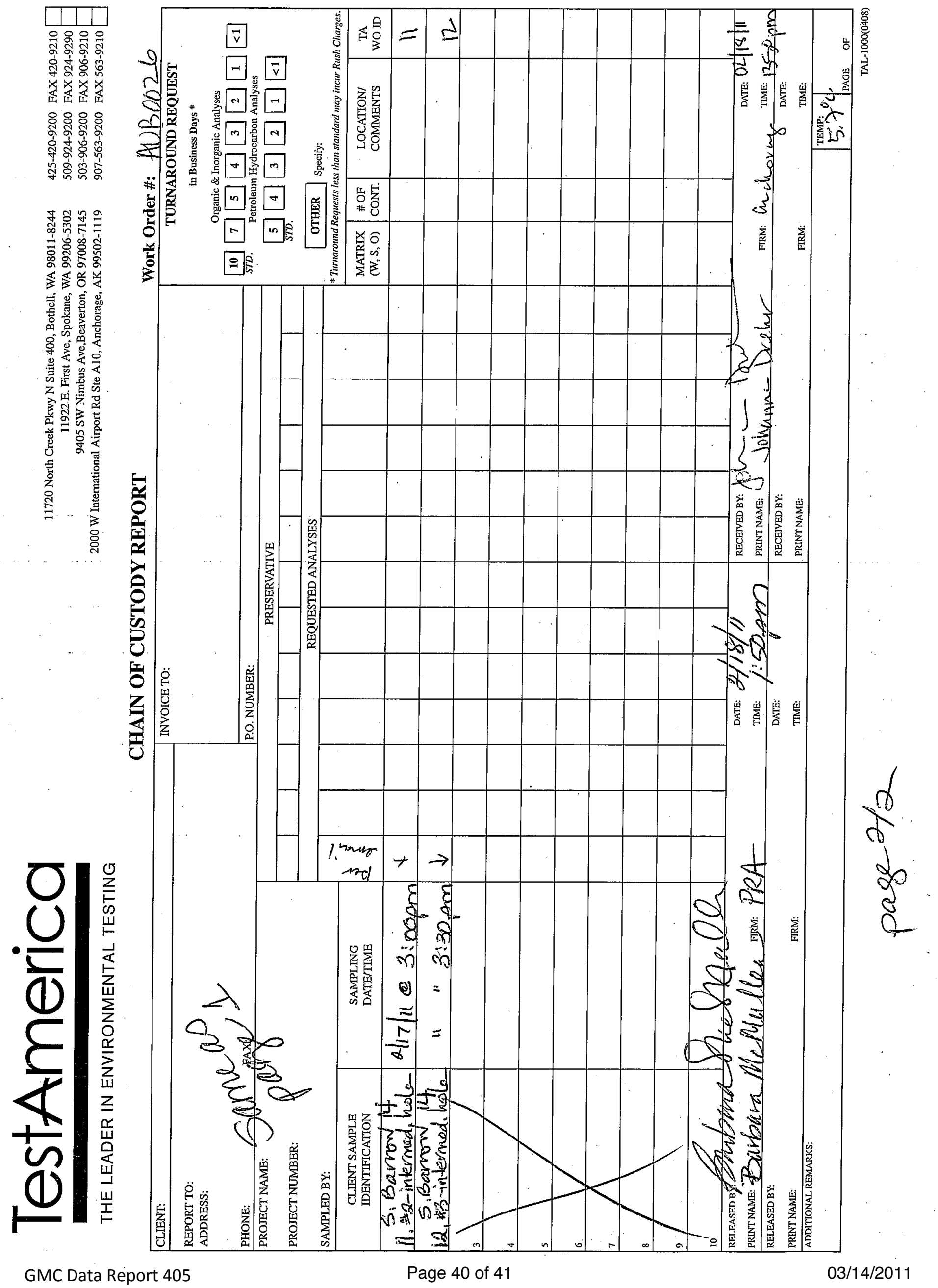




\section{Test America Anchorage Cooler Receipt Form}

(Army Corps. Conpliant)

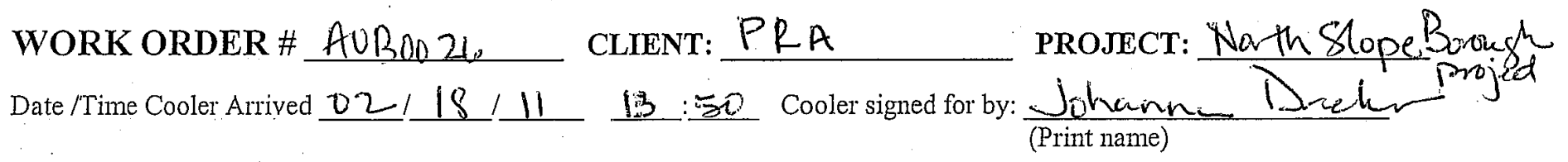

\section{Preliminary Examination Phase:}

Date cooler opened: $\square$ same as date received or

Cooler opened by (print) Johanne Driha

1. Delivered by $\square$ ALASKA AIRLINES $\square$ Fed-EX $\square$ UPS $\square$ NAC $\square$ LYNDEN EICLIENT

(sign)

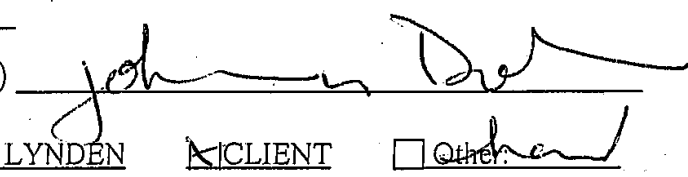

Shipment Tracking \# if applicable

NA

2. Number of Custody Seals

Signed by

Were custody seals unbroken and intact on arrival? (include copy of shipping papers in file)

$\mid \begin{aligned} & \text { (include copy of shipping papers in file) } \\ & \square \text { Yes }\end{aligned}$

3. Were custody papers sealed in a plastic bag?

$x$ Yes

$\square$ No

4. Were custody papers filled out properly (ink, signed, etc.)?

$\nabla$ Yes

$\square$ No

5. Did you sign the custody papers in the appropriate place?

$\nabla Y$ Yes

$\square$ N̦o

6. Was ice used? $\mathbb{X}$ Yes $\square$ No Type of ice: $\square$ blue ice $\square$ gel ice $\square$ real ice $\square$ dry ice condition of Ice: hating

Temperature by Digi-Thermo Probe $5.7{ }^{\circ} \mathrm{C}$ Thermometer \# rect 5 Acceptance Criteria: $0-6^{\circ} \mathrm{C}$

7. Packing in Cooler: \bubble wrap $\square$ styrofoam. \cardboard $\square$ Other:

8. Did samples anive in plastic bags?

$\square$ Yes

[ $]$ No

9. Did all bottles arrive unbroken, and with labels in good condition? $\varangle$ Yes

$\square$ No

10. Are all bottle labels complete (ID, date, time, etc.)

冈Yes

$\square$ No

11. Do bottle labels and Chain of Custody agree?

Yes

$\square$ No

12. Are the containers and preservatives correct for the tests indicated? $\mathbf{X} Y$ es

$\square$ No

13. Conoco Phillips, Alyeska, BP H2O samples only: $\mathrm{pH}<2$ ?

$\square$ Yes

$\square$ No

N/A

14. Is there adequate volunie for the tests requested?

XYes

$\square$ No

15. Were VOA vials free of bubbles?

区 N/A

$\square$ Yes

$\square$ No

If "NO" which containers contained "head space" or bubbles?

\section{Log-in Phase:}

Date of sample log-in $02,22,11$

Samples logged in by (print) Johanine Dreler (sign)

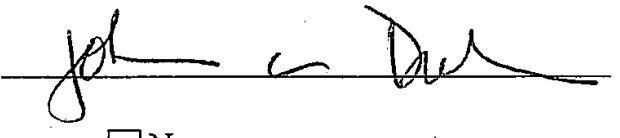

1. Was project identifiable from custody papers?

2. Do Tum Around Times and Due Dates agree?

ZXYes

$\square$ No

3. Was the Project Manager notified of status?

ד-Yes

$\square$ No

4. Was the Lab notified of status?

†TYes

$\square$ No

5. Was the COC scanned and copied?

XYes

$\square$ No

YYes

$\square$ No 